\title{
Testing relevant hypotheses in functional time series via self-normalization
}

\author{
Holger Dette, Kevin Kokot \\ Ruhr-Universität Bochum \\ Fakultät für Mathematik \\ Bochum, Germany \\ e-mail: \{holger.dette, kevin.kokot\}@rub.de
}

\author{
Stanislav Volgushev \\ University of Toronto \\ Department of Statisical Sciences \\ Toronto, Canada \\ email: stanislav.volgushev@utoronto.ca
}

February 21, 2020

\begin{abstract}
In this paper we develop methodology for testing relevant hypotheses about functional time series in a tuning-free way. Instead of testing for exact equality, for example for the equality of two mean functions from two independent time series, we propose to test the null hypothesis of no relevant deviation. In the two sample problem this means that an $L^{2}$-distance between the two mean functions is smaller than a pre-specified threshold. For such hypotheses self-normalization, which was introduced by Shao (2010) and Shao and Zhang (2010) and is commonly used to avoid the estimation of nuisance parameters, is not directly applicable. We develop new self-normalized procedures for testing relevant hypotheses in the one sample, two sample and change point problem and investigate their asymptotic properties. Finite sample properties of the proposed tests are illustrated by means of a simulation study and data examples. Our main focus is on functional time series, but extensions to other settings are also briefly discussed.
\end{abstract}

Keywords: self-normalization, functional time series, two sample problems, change point analysis, CUSUM, relevant hypotheses

\section{Introduction}

Statistics for functional data has found considerable interest in the last twenty years as documented in the various monographs by Ramsay and Silverman (2005), Ferraty and Vieu (2010) and Horváth and Kokoszka (2012) among others. The available methodology includes explorative tools such as shift and feature registration, warping or principal components, and methods for 
statistical inference such as testing of hypotheses and change point analysis. In this context a large portion of the literature attacks the problem of hypotheses testing by considering hypotheses of the form

$$
H_{0}: d=0 \text { versus } H_{1}: d \neq 0
$$

where $d$ is a real valued parameter such as the norm of the mean function in one sample or the norm of the difference of two mean functions or two covariance operators from two samples. For example Hall and Van Keilegom (2007) study the effect of smoothing when converting discrete observations into functional data, Horváth et al. (2009) compare linear operators in two functional regression models, Benko et al. (2009) propose functional principal component analysis (FPCA) for two sample inference while Panaretos et al. (2010) and Fremdt et al. (2013) consider a test for the equality of covariance operators. More recently Horváth et al. (2013) suggest tests for the comparison of two mean functions from temporally dependent curves under model-free assumptions and Pomann et al. (2016) compare the distributions of two samples by methods which are based on FPCA. Another important research area in functional data analysis is change point detection and we refer to Berkes et al. (2009), Hörmann and Kokoszka (2010), Aston and Kirch (2012a), Zhang et al. (2011), Horváth et al. (2014), Bucchia and Wendler (2017) among others who investigate change point problems from various perspectives.

Several authors consider methods for independent data. In this case the quantiles for corresponding tests can be easily obtained by asymptotic theory as the unknown quantities in the limit distribution of the test statistics can be reliably estimated (for example the asymptotic variance of a standardized mean). However, for functional samples exhibiting temporal dependence, the asymptotic distribution of many commonly used tests statistics involves the long-run variance, which makes the statistical inference substantially more difficult. Several authors propose to estimate the long-run variance [see Kokoszka (2012) or Horváth et al. (2013) among others], but the commonly used estimators depend on regularization parameters. As alternative, bootstrap methods can be applied to obtain critical values and we refer to Benko et al. (2009), Cuevas et al. (2006), Zhang et al. (2010), Bucchia and Wendler (2017) and Paparoditis and Sapatinas (2016) among many others. A third method to obtain (asymptotically) pivotal test statistics is the concept of self-normalization, which was introduced in the seminal papers of Shao (2010) for the construction of confidence intervals and Shao and Zhang (2010) for change point analysis. More recently it has been developed further for the specific needs of functional data by Zhang et al. (2011) and Zhang and Shao (2015) [see also Shao (2015) for a recent review].

This list of references is by no means complete but a common feature of all of these references is that they usually address hypotheses of the form (1.1), which we call "classical" hypotheses in the following discussion. However, in many applications one might not be interested in detecting very small deviations of the parameter $d$ from 0 (often the researcher even knows that $d$ is not exactly equal to 0, before any experiments have been carried out). For example, in change point detection a modification of the statistical analysis for prediction might not be necessary if the difference between the parameters before and after the change point is rather small. This 
discussion may be viewed as a particular case of the common bias variance trade-off in statistics. Therefore we argue that one should carefully think about the size of the difference in which one is interested. In particular we propose to replace the hypotheses (1.1) by the hypotheses of relevant differences, that is

$$
H_{0}: d \leq \Delta \text { versus } H_{1}: d>\Delta
$$

where $\Delta$ is a pre-specified constant representing the "maximal" value for the parameter $d$, which can be accepted as not scientifically significant. If the null hypothesis in $(1.2)$ holds we speak of a null of no relevant difference. This formulation of the testing problem requires the specification of the threshold $\Delta>0$, which depends on the specific application. "Classical" hypotheses tests simply use $\Delta=0$, but we argue that from a practical point of view it might be very reasonable to think about this choice more carefully and to define the size of the change in which one is really interested from a scientific viewpoint.

We also note that the formulation of the testing problem in the form 1.2 avoids the consistency problem mentioned in Berkson (1938), that is: any consistent test will detect any arbitrary small change in the parameters if the sample size is sufficiently large. Moreover, by interchanging the hypotheses, that is considering the hypotheses of equivalence

$$
H_{0}: d>\Delta \text { versus } H_{1}: d \leq \Delta \text {, }
$$

one is able to decide for a "small parameter" $d$ at a controlled type I error (for example that the norm $d$ of the difference between the mean functions of two samples is smaller than a given threshold). Hypotheses of the form (1.2) and (1.3) are called precise hypotheses or relevant hypotheses in the literature [see Berger and Delampady (1987)] and are frequently used in biostatistics. We refer to Chow and Liu (1992) and Wellek (2010) for more details and applications.

In this paper we discuss the problem of testing relevant hypotheses in the context of functional dependent data. We are particularly interested in methods based on self-normalization in order to avoid estimation of the long-run variance or resampling methods. The construction of efficient long-run variance estimates and resampling techniques is more difficult for testing relevant hypotheses, because - in contrast to "classical" hypotheses - the null hypothesis usually corresponds to an infinite dimensional set (for example the set of mean function with squared $L^{2}$-norm less or equal than $\Delta$ ).

For this purpose we modify the classical approaches to self-normalization based testing proposed by Shao (2010) and Shao and Zhang (2010) in order to make them applicable for testing relevant hypotheses. Zhang et al. (2011) and Zhang and Shao (2015) also use the concept of selfnormalization to develop statistical methodology for functional data analysis. In particular they construct tests for a change in the mean function and in the lag-1 autocovariance operator and for comparing the covariance operators and associated eigenvalues or eigenvectors from two samples. The main differences between their approach and the methods presented here are the following. First, these references do not consider the problem of testing relevant hypotheses, but deal with "classical" hypotheses of the form (1.1). Thus the present paper addresses a different statistical 
problem, where currently available methods are not applicable. Second, their approach is based on a dimension reduction projecting the functions on a finite dimensional vector (for example principal components), which is then used for the subsequent statistical inference using common self-normalization techniques. In contrast to their work we are able to develop a self-normalized test for the problem of testing relevant hypotheses of the form 1.2 , which does not require dimension reduction. For this purpose the common concepts of self-normalization have to be further extended. This modification is of independent interest besides the field of functional data analysis and applicable in many other problems.

The remaining part of this paper is organized as follows. Our basic idea is explained in Section 2 for the one and two sample case, where it is most transparent. Roughly speaking, we construct an asymptotic confidence interval for the parameter $d$ to obtain tests for hypotheses of the form (1.2) and (1.3). In Section 3 we address the problem of relevant change point analysis by the new way of self-normalization; here an additional challenge arises from the fact that the change point location is unknown and needs to be estimated. While the methodology in Section 2 and 3 refers to statistical inference for mean functions we illustrate in Section 4 how those ideas can be extended to inference for covariance operators. Some finite sample results are presented in Section 5, where we also illustrate the proposed methodology on two data examples. Here we also provide brief discussion of self-normalization and estimation of the long-run variance in the context of testing relevant hypotheses. Finally, in an online supplement we present additional finite sample results (Section A), give the proofs of our results (Section B) and discuss extensions beyond functional time series (Section C).

\section{Relevant hypotheses and self normalization}

Let $T$ be a compact set in $\mathbb{R}^{d}$ and let $L^{2}(T)$ denote the Hilbert space of square integrable functions on the set $T$ with the usual inner product $\langle\cdot, \cdot\rangle$ and corresponding norm $\|\cdot\|$.

\subsection{One sample problems}

Let $\left\{X_{n}\right\}_{n \in \mathbb{Z}}$ denote a strictly stationary functional time series where the random variables $X_{n}$ are elements in $L^{2}(T)$ (with expectation $\mu:=\mathbb{E}\left[X_{1}\right] \in L^{2}(T)$, see Section 2.1 in Bücher et al. (2019) for a detailed discussion of expected values in Hilbert spaces). For the sake of simplicity we will assume that $T=[0,1]$, but all methods proposed in this paper can be generalized to other subsets of $\mathbb{R}^{d}$. To avoid confusion between the interval $[0,1]$ corresponding to $\lambda$, which defines the sub-sample $X_{1}, \ldots, X_{\lfloor n \lambda\rfloor}$ and the interval $T=[0,1]$, we write $T$ for the interval $[0,1]$ belonging to the argument $t$ of $X_{n}$. Based on a sample $X_{1}, \ldots, X_{n}$ we are interested in relevant hypotheses regarding the parameter $d=\int_{T} \mu^{2}(t) d t$, that is

$$
H_{0}: \int_{T} \mu^{2}(t) d t \leq \Delta \quad \text { versus } \quad H_{1}: \int_{T} \mu^{2}(t) d t>\Delta .
$$


Define the partial sums

$$
S_{n}(t, \lambda):=\frac{1}{n} \sum_{j=1}^{\lfloor n \lambda\rfloor} X_{j}(t), \quad \lambda \in[0,1]
$$

then, under suitable assumptions, the statistic $\int_{T} S_{n}^{2}(t, 1) d t$ is a consistent estimator of $\int_{T} \mu^{2}(t) d t$. Consequently a test for the hypotheses (2.1) is obtained by rejecting the null hypothesis of no relevant difference for large values of

$$
\hat{\mathbb{T}}_{n}=\int_{T} S_{n}^{2}(t, 1) d t
$$

It will be shown in the proof of Theorem 2.1 that under some technical assumptions the asymptotic distribution of an appropriately standardized version of $\hat{\mathbb{T}}_{n}$ takes the form

$$
\sqrt{n}\left(\hat{\mathbb{T}}_{n}-\int_{T} \mu^{2}(t) d t\right) \stackrel{\mathcal{D}}{\rightarrow} \mathcal{N}\left(0, \tau^{2}\right)
$$

with long-run variance

$$
\tau^{2}=4 \int_{T} \int_{T} \mu(s) \mu(t) C(s, t) d s d t
$$

where

$$
C(s, t)=\operatorname{Cov}\left(X_{0}(s), X_{0}(t)\right)+\sum_{\ell=1}^{\infty} \operatorname{Cov}\left(X_{0}(s), X_{\ell}(t)\right)+\sum_{\ell=1}^{\infty} \operatorname{Cov}\left(X_{0}(s), X_{-\ell}(t)\right)
$$

is the long-run covariance operator of the process $\left\{X_{n}\right\}_{n \in \mathbb{Z}}$. Here we note that the above weak convergence is also true when $\mu \equiv 0$, in which case the limit is a degenerate normal distribution with a point mass at zero. Unfortunately, the long-run variance $\tau^{2}$ is difficult to estimate in practice. This motivates us to adopt a self-normalization approach which avoids direct estimation of $\tau^{2}$. To be more precise let $\nu$ denote a probability measure on the interval $(0,1)$ and define

$$
\hat{\mathbb{V}}_{n}:=\left(\int_{0}^{1}\left[\int_{T} S_{n}^{2}(t, \lambda) d t-\lambda^{2} \int_{T} S_{n}^{2}(t, 1) d t\right]^{2} \nu(d \lambda)\right)^{1 / 2} .
$$

As we will show later we have

$$
\left(\sqrt{n}\left(\hat{\mathbb{T}}_{n}-d\right), \sqrt{n} \hat{\mathbb{V}}_{n}\right) \stackrel{\mathcal{D}}{\rightarrow}\left(\tau \mathbb{B}(1), \tau\left(\int_{0}^{1} \lambda^{2}(\mathbb{B}(\lambda)-\lambda \mathbb{B}(1))^{2} \nu(d \lambda)\right)^{1 / 2}\right),
$$

where $\mathbb{B}$ denotes a standard Brownian motion on the interval $[0,1]$. In particular, this implies that, in the case $\tau \neq 0$, the ratio $\left(\hat{\mathbb{T}}_{n}-d\right) / \hat{\mathbb{V}}_{n}$ converges to a pivotal distribution. This suggests that a test for 2.1) can be constructed by rejecting the null hypothesis of no relevant difference in (2.1), whenever

$$
\hat{\mathbb{T}}_{n}>\Delta+q_{1-\alpha}(\mathbb{W}) \hat{\mathbb{V}}_{n}
$$




\begin{tabular}{c|ccc} 
& $99 \%$ & $95 \%$ & $90 \%$ \\
\hline \hline 1$)$ & 18.257 & 10.998 & 7.855 \\
$2)$ & 16.081 & 10.530 & 7.619 \\
$3)$ & 16.282 & 10.583 & 7.662
\end{tabular}

Table 1: Simulated quantiles (based on 1000 replications) of the distribution of the statistic $\mathbb{W}$ defined by (2.9), where $\nu$ is the discrete uniform distribution supported on the points 1) $\lambda_{i}=i / 5$ $(i=1, \ldots, 4)$, 2) $\lambda_{i}=i / 20(i=1, \ldots, 19)$ and 3) $\lambda_{i}=i / 100(i=1, \ldots, 99)$.

where $q_{1-\alpha}(\mathbb{W})$ denotes the $(1-\alpha)$-quantile of the distribution of the pivotal random variable

$$
\mathbb{W}:=\frac{\mathbb{B}(1)}{\left(\int_{0}^{1} \lambda^{2}(\mathbb{B}(\lambda)-\lambda \mathbb{B}(1))^{2} \nu(d \lambda)\right)^{1 / 2}} .
$$

It is worthwhile to mention that the distribution of $\mathbb{W}$ is not the same as the one in previous work on self-normalization [see for example Shao (2010) or Shao (2015)] and quantiles of this distribution need to be simulated first. In Table 2.1 we display quantiles of this distribution, where $\nu$ is the discrete uniform distribution supported on the points $\lambda_{i}=i / 5(i=1, \ldots, 4)$, on the points $\lambda_{i}=i / 20(i=1, \ldots, 19)$ and on the points $\lambda_{i}=i / 100(i=1, \ldots, 99)$, respectively.

Next we prove that the decision rule in 2.8 indeed provides an asymptotic level $\alpha$ test. For this purpose we make the following assumptions [see also Berkes et al. (2013); Horváth et al. (2014)]:

(A1) For all $j \in \mathbb{Z}$ we have $X_{j}=\mu+\eta_{j}$, where $\left(\eta_{j}\right)_{j \in \mathbb{Z}}$ is a centered error process which satisfies (A2)-(A4).

(A2) $\left(\eta_{j}\right)_{j \in \mathbb{Z}}$ is a sequence of Bernoulli shifts, that is: there exists a measurable space, say $\mathcal{S}$ and a function $f: \mathcal{S}^{\infty} \longrightarrow L^{2}([0,1])$ such that

$$
\eta_{j}=f\left(\varepsilon_{j}, \varepsilon_{j-1}, \ldots\right) \quad \text { for all } j \in \mathbb{Z},
$$

where $\left(\varepsilon_{j}\right)_{j \in \mathbb{Z}}$ is a sequence of i.i.d $\mathcal{S}$-valued functions, such that $\varepsilon_{j}(t)=\varepsilon_{j}(t, \omega)$ is jointly measurable $(j \in \mathbb{Z})$.

(A3) $\mathbb{E}\left\|\eta_{j}\right\|^{2+\psi}<\infty$ for some $\psi \in(0,1)$.

(A4) The sequence $\left(\eta_{j}\right)_{j \in \mathbb{Z}}$ can be approximated by $\ell$-dependent sequences $\left(\eta_{j, \ell}\right)_{j \in \mathbb{Z}}$ in the sense that for some $\kappa>2+\psi$

$$
\sum_{\ell=1}^{\infty}\left(\mathbb{E}\left\|\eta_{0}-\eta_{0, \ell}\right\|^{2+\psi}\right)^{1 / \kappa}<\infty
$$


where $\eta_{j, \ell}$ is defined by

$$
\begin{aligned}
& \eta_{j, \ell}=f\left(\varepsilon_{j}, \varepsilon_{j-1}, \ldots \varepsilon_{j-\ell+1}, \varepsilon_{j, \ell}^{*}\right) \\
& \varepsilon_{j, \ell}^{*}=\left(\varepsilon_{j, \ell, j-\ell}^{*}, \varepsilon_{j, \ell, j-\ell-1}^{*}, \ldots\right)
\end{aligned}
$$

and the random variables $\varepsilon_{j, \ell, k}^{*}$ are i.i.d. copies of $\varepsilon_{0}$, and independent of the sequence $\left(\varepsilon_{j}\right)_{j \in \mathbb{Z}}$.

Theorem 2.1 Assume that $\Delta>0$. Under the assumptions (A1)-(A4) the test decision given in (2.8) satisfies

$$
\lim _{n \rightarrow \infty} \mathbb{P}\left(\hat{\mathbb{T}}_{n}>\Delta+q_{1-\alpha}(\mathbb{W}) \hat{\mathbb{V}}_{n}\right)= \begin{cases}0 & \text { if } \int_{T} \mu^{2}(t) d t<\Delta \\ \alpha & \text { if } \int_{T} \mu^{2}(t) d t=\Delta \text { and } \tau^{2}>0 \\ 1 & \text { if } \int_{T} \mu^{2}(t) d t>\Delta\end{cases}
$$

A detailed proof of Theorem 2.1 is given in Section B.1.1. In what follows we provide an informal overview of the main steps in the proof. If $\int \mu^{2}(t) d t \neq 0$ and assumptions (A1) - (A4) hold, it can be shown that

$$
\left\{\sqrt{n}\left(\int_{T} S_{n}^{2}(t, \lambda) d t-\lambda^{2} \int_{T} \mu^{2}(t) d t\right)\right\}_{\lambda \in[0,1]} \rightsquigarrow\{\lambda \tau \mathbb{B}(\lambda)\}_{\lambda \in[0,1]},
$$

where the symbol $\rightsquigarrow$ means weak convergence in $\ell^{\infty}([0,1])$ and $\tau^{2}$ is defined in (2.4). Now an application of the continuous mapping theorem directly yields the joint weak convergence (2.7). This implies the statement of Theorem 2.1 when $\int \mu^{2}(t) d t>0$ after some simple computations. If $\int \mu^{2}(t) d t=0$ it is possible to prove that $\hat{\mathbb{T}}_{n}=o_{\mathbb{P}}(1), \hat{\mathbb{V}}_{n}=o_{\mathbb{P}}(1)$. This implies

$$
\lim _{n \rightarrow \infty} \mathbb{P}\left(\hat{\mathbb{T}}_{n}>\Delta+q_{1-\alpha}(\mathbb{W}) \hat{\mathbb{V}}_{n}\right)=\lim _{n \rightarrow \infty} \mathbb{P}\left(o_{\mathbb{P}}(1)>\Delta\right)=0,
$$

where we used that $\Delta>0$ is fixed.

Remark 2.1 In general, the rejection rule in (2.8) does not lead to an asymptotic level $\alpha$ test when $\Delta=0$. To see this note that for $\Delta=0$ the null hypothesis contains only one point $\mu \equiv 0$ and we reject the null when $\hat{\mathbb{T}}_{n} / \hat{\mathbb{V}}_{n}>q_{1-\alpha}(\mathbb{W})$. However, a slight extension of the arguments given in the proof of (2.11) shows that for $\mu \equiv 0$ we have

$$
\frac{\hat{\mathbb{T}}_{n}}{\hat{\mathbb{V}}_{n}} \stackrel{\mathcal{D}}{\rightarrow} \tilde{\mathbb{W}}:=\frac{\int_{T} \Gamma^{2}(t, 1) d t}{\left\{\int_{0}^{1}\left(\int_{T} \Gamma^{2}(t, \lambda) d t-\lambda^{2} \int_{T} \Gamma^{2}(t, 1) d t\right)^{2} \nu(d \lambda)\right\}^{1 / 2}}
$$

where $\Gamma(t, \lambda)$ is a centered Gaussian process with covariance function

$$
\operatorname{Cov}\left(\Gamma(t, \lambda), \Gamma\left(s, \lambda^{\prime}\right)\right)=\left(\lambda \wedge \lambda^{\prime}\right) C(s, t),
$$

where $C$ is the long-run covariance operator defined in 2.5 . The distribution of $\tilde{\mathbb{W}}$ does not match that of $\mathbb{W}$ and is not pivotal. Hence a test based on rejecting $H_{0}: \mu=0$ using the decision rule 2.8 will not have asymptotic level $\alpha$. 
Remark 2.2 A test for the hypotheses of equivalence

$$
H_{0}: \int_{T} \mu^{2}(t) d t>\Delta \quad \text { versus } \quad H_{1}: \int_{T} \mu^{2}(t) d t \leq \Delta
$$

can be obtained similarly. The null hypothesis of a relevant difference in $(2.12)$ is rejected, when

$$
\hat{\mathbb{T}}_{n} \leq \Delta+q_{\alpha}(\mathbb{W}) \hat{\mathbb{V}}_{n}
$$

where $\hat{\mathbb{T}}_{n}$ and $\hat{\mathbb{V}}_{n}$ are defined in $(2.3)$ and $(2.6)$, respectively and $q_{\alpha}(\mathbb{W})$ is the $\alpha$-quantile of the distribution of $\mathbb{W}$ defined in 2.9 . Similar arguments as given in the proof of Theorem 2.1 show that this test is an asymptotic level $\alpha$ and consistent test for the hypotheses (2.12), that is

$$
\lim _{n \rightarrow \infty} \mathbb{P}\left(\hat{\mathbb{T}}_{n} \leq \Delta+q_{\alpha}(\mathbb{W}) \hat{\mathbb{V}}_{n}\right)= \begin{cases}1 & \text { if } \int_{T} \mu^{2}(t) d t<\Delta \\ \alpha & \text { if } \int_{T} \mu^{2}(t) d t=\Delta \text { and } \tau^{2}>0 \\ 0 & \text { if } \int_{T} \mu^{2}(t) d t>\Delta\end{cases}
$$

The details are omitted for the sake of brevity.

Remark 2.3 As pointed out by a referee it is of interest to compare the test (2.8) based on self-normalization with a corresponding test using an estimate of the long-run variance. For this purpose note that such a test rejects the null hypothesis of no relevant difference (2.1), whenever

$$
\hat{\mathbb{T}}_{n}>\Delta+u_{1-\alpha} \frac{\hat{\tau}_{n}}{\sqrt{n}},
$$

where $u_{1-\alpha}$ is the $(1-\alpha)$-quantile of the standard normal distribution and $\hat{\tau}_{n}^{2}$ is an appropriate estimator of the long-run variance (2.4). In the case of one sample this is still relatively easy. For example, one could use

$$
\hat{\tau}_{n}^{2}=4 \int_{0}^{1} \int_{0}^{1} S_{n}(s, 1) S_{n}(t, 1) \hat{C}_{n}(s, t) d s d t,
$$

where $S_{n}(t, 1)$ is defined in 2.2 and $\hat{C}_{n}$ is an appropriate estimator of the long-run covariance operator. A numerical illustration of this approach in comparison with self-normalization can be found in Section 5.1.1.

\subsection{Two sample problems}

Throughout this section let $\left\{X_{n}\right\}_{n \in \mathbb{Z}},\left\{Y_{n}\right\}_{n \in \mathbb{Z}}$ denote two strictly stationary functional time series with values in $L^{2}(T)$. Assume that we observe finite stretches, say $X_{1}, \ldots, X_{m}$ and $Y_{1}, \ldots, Y_{n}$ from 
$\left\{X_{n}\right\}_{n \in \mathbb{Z}}$ and $\left\{Y_{n}\right\}_{n \in \mathbb{Z}}$. Denote by $\mu_{1}=\mathbb{E}\left[X_{1}\right]$ and $\mu_{2}=\mathbb{E}\left[Y_{1}\right]$ the corresponding mean functions, by $D(t)=\mu_{1}(t)-\mu_{2}(t)$ their difference and define the partial sum

$$
D_{m, n}(t, \lambda):=\frac{1}{m} \sum_{j=1}^{\lfloor m \lambda\rfloor} X_{j}(t)-\frac{1}{n} \sum_{j=1}^{\lfloor n \lambda\rfloor} Y_{j}(t) .
$$

From this definition we see that

$$
\mathbb{E}\left[D_{m . n}(t, \lambda)\right]=\lambda D(t)+O\left((m \wedge n)^{-1}\right) .
$$

For the sake of brevity we restrict ourselves to the problem of testing the relevant hypotheses

$$
H_{0}: \int_{T} D^{2}(t) d t \leq \Delta \quad \text { versus } \quad H_{1}: \int_{T} D^{2}(t) d t>\Delta,
$$

where $\Delta$ is a pre-specified threshold. A corresponding test for the hypotheses of equivalence can be derived along the lines given in Remark 2.2. Following the discussion in Section 2.1 we propose to reject the null hypothesis of no relevant difference in $(2.16)$, whenever

$$
\hat{\mathbb{D}}_{m, n}>\Delta+q_{1-\alpha}(\mathbb{W}) \hat{\mathbb{V}}_{m, n}
$$

where $q_{1-\alpha}(\mathbb{W})$ is the $(1-\alpha)$-quantile of the distribution of the random variable $\mathbb{W}$ in $(2.9)$. The statistics $\hat{\mathbb{D}}_{m, n}$ and $\hat{\mathbb{V}}_{m, n}$ are defined by

$$
\begin{aligned}
& \hat{\mathbb{D}}_{m, n}=\int_{T} D_{m, n}^{2}(t, 1) d t \\
& \hat{\mathbb{V}}_{m, n}=\left(\int_{0}^{1}\left[\int_{T} D_{m, n}^{2}(t, \lambda) d t-\lambda^{2} \int_{T} D_{m, n}^{2}(t, 1) d t\right]^{2} \nu(d \lambda)\right)^{1 / 2}
\end{aligned}
$$

respectively, where $\nu$ is a probability measure on the interval $(0,1)$. The asymptotic properties of this test procedure will be established under the following assumptions.

(B1) The sample sizes satisfy: $m \rightarrow \infty$ and $n \rightarrow \infty$ and $m /(m+n) \rightarrow \rho \in(0,1)$.

(B2) The processes $\left\{X_{n}\right\}_{n \in \mathbb{Z}}$ and $\left\{Y_{n}\right\}_{n \in \mathbb{Z}}$ are independent and satisfy assumptions (A1) - (A4) stated in Section 2.1 with $\mathbb{E}\left[X_{1}\right]=\mu_{1}, \mathbb{E}\left[Y_{1}\right]=\mu_{2}$.

We also define the quantity

$$
\tau_{D}^{2}=4 \int_{T} \int_{T} D(s) D(t)\left(\frac{1}{\rho} C_{X}(s, t)+\frac{1}{1-\rho} C_{Y}(s, t)\right) d s d t
$$

where $C_{X}$ and $C_{Y}$ are the long-run covariance operators corresponding to the processes $\left\{X_{n}\right\}_{n \in \mathbb{Z}}$ and $\left\{Y_{n}\right\}_{n \in \mathbb{Z}}$, respectively. 
Theorem 2.2 Assume that $\Delta>0$. Under assumptions (B1)-(B2) the test decision given in (2.17) satisfies

$$
\lim _{n \rightarrow \infty} \mathbb{P}\left(\hat{\mathbb{D}}_{m, n}>\Delta+q_{1-\alpha}(\mathbb{W}) \hat{\mathbb{V}}_{m, n}\right)= \begin{cases}0 & \text { if } \int_{T} D^{2}(t) d t<\Delta, \\ \alpha & \text { if } \int_{T} D^{2}(t) d t=\Delta \text { and } \tau_{D}^{2}>0 \\ 1 & \text { if } \int_{T} D^{2}(t) d t>\Delta .\end{cases}
$$

We note that similarly to the one-sample case it can be shown that the rejection rule in $(2.17)$ does not lead to an asymptotic level $\alpha$ test when $\Delta=0$.

\section{Remark 2.4}

(a) The statement in Theorem 2.2 continues to hold if the observations $X_{i}, Y_{i}$ are generated according to $X_{i}=\mu_{1}+f_{1}\left(\varepsilon_{i}, \varepsilon_{i-1, \ldots}\right), i=1, \ldots, n$ and $Y_{i}=\mu_{2}+f_{2}\left(\varepsilon_{n+i}, \varepsilon_{n+i-1, \ldots}\right), i=1, \ldots, m$ where $\left(\varepsilon_{j}\right)_{j \in \mathbb{Z}}$ denotes an i.i.d. sequence of $\mathcal{S}$-valued functions with the property that $\varepsilon_{j}(t, \omega)$ is jointly measurable as in $(\mathrm{A} 2)$ and $f_{1}, f_{2}: \mathcal{S}^{\infty} \rightarrow L^{2}([0,1])$ are functions such that the processes $\left(f_{1}\left(\varepsilon_{i}, \varepsilon_{i-1, \ldots}\right)\right)_{i \in \mathbb{Z}}$ and $\left(f_{2}\left(\varepsilon_{i}, \varepsilon_{i-1, \ldots}\right)\right)_{i \in \mathbb{Z}}$ satisfy conditions (A3) and (A4). This essentially corresponds to the setting discussed in Section 3 when the change point location is known.

(b) A test based on estimation of the long-run variance of the statistic $\hat{\mathbb{D}}_{m, n}$ can be constructed along the lines given in Remark 2.3. The details are omitted for the sake of brevity.

Remark 2.5 As pointed out by the Associate Editor the proposed way of self normalization is not unique and one could also think about alternative constructions. For instance, one could also use the statistics

$$
\begin{aligned}
& \hat{\mathbb{V}}_{m, n}^{\star}=\nu \text { - } \underset{\lambda \in[0,1]}{\operatorname{essup}}\left|\int_{T} D_{m, n}^{2}(t, \lambda) d t-\lambda^{2} \int_{T} D_{m, n}^{2}(t, 1) d t\right| \\
& \hat{\mathbb{V}}_{m, n}^{\star \star}=\int_{0}^{1}\left|\int_{T} D_{m, n}^{2}(t, \lambda) d t-\lambda^{2} \int_{T} D_{m, n}^{2}(t, 1) d t\right| \nu(d \lambda)
\end{aligned}
$$

in the decision rule $(2.8)$ if the quantile $q_{1-\alpha}(\mathbb{W})$ is replaced by the $(1-\alpha)$-quantile of the random variables

$$
\mathbb{W}^{\star}:=\frac{\mathbb{B}(1)}{\nu-\operatorname{ess} \sup _{\lambda \in[0,1]}\left|\lambda\left(\mathbb{B}(\lambda)-\lambda \mathbb{B}^{2}(1)\right)\right|}, \quad \mathbb{W}^{\star \star}:=\frac{\mathbb{B}(1)}{\int_{0}^{1}\left|\lambda\left(\mathbb{B}(\lambda)-\lambda \mathbb{B}^{2}(1)\right)\right| \nu(d \lambda)}
$$

respectively. The self-normalizing factors 2.20 and 2.21 might have some advantages for heavy-tailed data. However, it will be demonstrated in Section 5.2 that the finite sample properties of these two alternative tests are very similar to those of the test (2.17).

\section{Relevant change points in the mean function}

In this section we consider data that are generated from the following (triangular array) model

$$
X_{i}= \begin{cases}\mu+f_{1}\left(\varepsilon_{i}, \varepsilon_{i-1, \ldots}\right) & \text { if } i \leq N \theta_{0} \\ \mu+\delta+f_{2}\left(\varepsilon_{i}, \varepsilon_{i-1, \ldots}\right) & \text { if } i>N \theta_{0}\end{cases}
$$


Here $\mu, \delta$ denote deterministic but unknown elements in $L^{2}(T)$ and $\theta_{0} \in(0,1)$ is fixed but unknown. Moreover, $\left(\varepsilon_{j}\right)_{j \in \mathbb{Z}}$ denotes an i.i.d. sequence of $\mathcal{S}$-valued functions with the property that $\varepsilon_{j}(t, \omega)$ is jointly measurable as in (A2) and $f_{1}, f_{2}: \mathcal{S}^{\infty} \rightarrow L^{2}(T)$ are functions such that the processes $\left(f_{1}\left(\varepsilon_{i}, \varepsilon_{i-1, \ldots}\right)\right)_{i \in \mathbb{Z}}$ and $\left(f_{2}\left(\varepsilon_{i}, \varepsilon_{i-1, \ldots}\right)\right)_{i \in \mathbb{Z}}$ satisfy conditions (A3) and (A4). This setting is general enough to allow for the whole distribution of the observed functional data to change together with their mean.

We aim to construct a test for the relevant hypothesis

$$
H_{0}: \int_{T} \delta^{2}(t) d t \leq \Delta \quad \text { versus } \quad H_{1}: \int_{T} \delta^{2}(t) d t>\Delta
$$

where $\Delta$ is a pre-specified threshold. Note that for known $\theta_{0}$ a test for $H_{0}$ can be constructed in a similar fashion as in Section 2.2. In this section, we will prove that replacing the known change point by an estimator also leads to an asymptotic level $\alpha$ test for the hypotheses in (3.2). To this end we fix a trimming parameter $\varepsilon \in[0,1 / 2)$ and define the estimator of the unknown change point $\theta_{0}$ as

$$
\hat{\theta}:=\frac{1}{N} \operatorname{argmax}_{\lfloor N \varepsilon\rfloor+1 \leq k \leq N-\lfloor N \varepsilon\rfloor} \hat{f}(k),
$$

where $\hat{f}(0)=\hat{f}(N)=0$ and for $k=1, \ldots, N-1$

$$
\hat{f}(k):=\frac{k}{N}\left(1-\frac{k}{N}\right) \int_{T}\left(\frac{1}{k} \sum_{j=1}^{k} X_{j}(t)-\frac{1}{N-k} \sum_{j=k+1}^{N} X_{j}(t)\right)^{2} d t .
$$

Our first result shows that the estimator $\hat{\theta}$ is consistent.

Proposition 3.1 If the data is generated according to model (3.1), $\int \delta^{2}(t) d t>0, \theta_{0} \in(\varepsilon, 1-\varepsilon)$, and the assumptions described right below (3.1) are satisfied, then

$$
\hat{\theta}=\theta_{0}+o_{\mathbb{P}}\left(N^{-1 / 2}\right) .
$$

Next we introduce the test statistic. For arbitrary $\theta \in[1 / N, 1)$ define

$$
D_{N}^{c p}(t, \lambda, \theta):=\frac{1}{\lfloor N \theta\rfloor} \sum_{j=1}^{\lfloor\lambda\lfloor\theta N\rfloor\rfloor} X_{j}(t)-\frac{1}{N-\lfloor N \theta\rfloor} \sum_{j=\lfloor\theta N\rfloor+1}^{\lfloor\theta N\rfloor+\lfloor\lambda(N-\lfloor\theta N\rfloor)\rfloor} X_{j}(t) .
$$

Following the developments in Section 2.2 let

$$
\begin{aligned}
\hat{\mathbb{D}}_{N}^{c p} & =\int_{T} D_{N}^{c p}(t, 1, \hat{\theta})^{2} d t \\
\hat{\mathbb{V}}_{N}^{c p} & =\left(\int_{0}^{1}\left[\int_{T} D_{N}^{c p}(t, \lambda, \hat{\theta})^{2} d t-\lambda^{2} \int_{T} D_{N}^{c p}(t, 1, \hat{\theta})^{2} d t\right]^{2} \nu(d \lambda)\right)^{1 / 2}
\end{aligned}
$$


respectively, where $\nu$ is a probability measure on the interval $(0,1)$. The test for $H_{0}$ takes the form

$$
\hat{\mathbb{D}}_{N}^{c p}>\Delta+q_{1-\alpha}(\mathbb{W}) \hat{\mathbb{V}}_{N}^{c p}
$$

where $q_{1-\alpha}(\mathbb{W})$ is the $(1-\alpha)$-quantile of the distribution of the random variable $\mathbb{W}$ in $(2.9)$. This test decision is justified in the following theorem. For its precise statement we define the quantity

$$
\tau_{\delta, \theta_{0}}^{2}=4 \int_{T} \int_{T} \delta(s) \delta(t)\left(\frac{1}{\theta_{0}} K_{1}(s, t)+\frac{1}{1-\theta_{0}} K_{2}(s, t)\right) d s d t
$$

where for $j=1,2$

$$
K_{j}(s, t)=\sum_{h \in \mathbb{Z}} \operatorname{Cov}\left(\eta_{0}^{(j)}(s), \eta_{h}^{(j)}(t)\right), \quad(j=1,2)
$$

is the long-run covariance kernel of $\left\{\eta_{i}^{(j)}\right\}_{i \in \mathbb{Z}}=\left\{f_{j}\left(\varepsilon_{i}, \varepsilon_{i-1, \ldots}\right)\right\}_{i \in \mathbb{Z}}$.

Theorem 3.1 Assume $\Delta>0$. If the data is generated according to model (3.1), $\theta_{0} \in(\varepsilon, 1-\varepsilon)$, and the assumptions described right below (3.1) hold, then the test decision in (3.8) satisfies

$$
\lim _{n \rightarrow \infty} \mathbb{P}\left(\hat{\mathbb{D}}_{N}^{c p}>\Delta+q_{1-\alpha}(\mathbb{W}) \hat{\mathbb{V}}_{N}^{c p}\right)= \begin{cases}0 & \text { if } \int_{T} \delta^{2}(t) d t<\Delta, \\ \alpha & \text { if } \int_{T} \delta^{2}(t) d t=\Delta \text { and } \tau_{\delta, \theta_{0}}^{2}>0, \\ 1 & \text { if } \int_{T} \delta^{2}(t) d t>\Delta .\end{cases}
$$

Similarly to the one-sample and two-sample case, the rejection rule in $(3.8)$ does not lead to an asymptotic level $\alpha$ test when $\Delta=0$.

The proof of Proposition 3.1 and Theorem 3.1 is technically difficult and deferred to Section B.2, but the main idea is as follows. A straightforward calculation shows that the processes $\hat{\mathbb{D}}_{N}^{c p}$ and $\hat{\mathbb{V}}_{N}^{c p}$ in $(3.6)$ and $(3.7)$ are continuous functionals of the process

$$
\mathbb{Z}_{N}(\lambda, \hat{\theta})=\sqrt{N} \int_{T}\left(D_{N}^{c p}(t, \lambda, \hat{\theta})^{2}-\lambda^{2} \delta(t)^{2}\right) d t
$$

Using Proposition 3.1 it can be shown that

$$
\sup _{\lambda \in[0,1]}\left|\mathbb{Z}_{N}\left(\lambda, \theta_{0}\right)-\mathbb{Z}_{N}(\lambda, \hat{\theta})\right|=o_{\mathbb{P}}(1),
$$

where $\theta_{0}$ is the true change point. We can then establish the weak convergence

$$
\left\{\mathbb{Z}_{N}\left(\lambda, \theta_{0}\right)\right\}_{\lambda \in[0,1]} \rightsquigarrow\left\{\lambda \tau_{\delta, \theta_{0}} \mathbb{B}(\lambda)\right\}_{\lambda \in[0,1]},
$$

where $\tau_{\delta, \theta}^{2}$ defined in 3.9 . Using the continuous mapping theorem we then find

$$
\frac{\hat{\mathbb{D}}_{N}^{c p}}{\hat{\mathbb{V}}_{N}^{c p}} \stackrel{\mathcal{D}}{\rightarrow} \mathbb{W},
$$

where the random variable $\mathbb{W}$ is defined in $(2.9)$. When $\int_{T} \delta^{2}(t) d t>0$, the assertion of Theorem 3.1 now follows directly. In the remaining case $\int_{T} \delta^{2}(t) d t=0$ one can show $\hat{\mathbb{D}}_{N}^{c p}=$ $o_{\mathbb{P}}(1), \mathbb{\mathbb { V }}_{N}^{c p}=o_{\mathbb{P}}(1)$ and the assertion follows with the same arguments as given in Section 2.1. 
Remark 3.1 In this remark we briefly explain how - in principle - a test can be constructed using an appropriate estimate of the long-run variance of the statistic $\hat{\mathbb{D}}_{N}^{c p}$ in $(3.6)$. A careful inspection of the proof of Theorem 3.1, (see in particular the discussion at the end of the proof of (B.31) in the online supplement) shows that

$$
\sqrt{N}\left(\hat{\mathbb{D}}_{N}^{c p}-\int_{T} \delta^{2}(t) d t\right) \stackrel{\mathcal{D}}{\longrightarrow} \mathcal{N}\left(0, \tau_{\delta, \theta_{0}}^{2}\right)
$$

where the asymptotic variance is defined in $(3.9)$. If $\hat{\tau}_{N}^{2}$ denotes an estimator of $\tau_{\delta, \theta_{0}}^{2}$, an asymptotic level $\alpha$ test is obtained by rejecting the null hypothesis in $(3.2)$, whenever

$$
\hat{\mathbb{D}}_{N}^{c p}>\Delta+\frac{\hat{\tau}_{N}}{\sqrt{N}} u_{1-\alpha}
$$

One possibility to estimate $\tau_{\delta, \theta_{0}}^{2}$ is to replace $\theta_{0}$ by the estimator $\hat{\theta}$ defined in (3.3) and to use appropriate plug-in estimators for the covariance kernels $K_{j}$ and unknown difference $\delta$ based on the sub-samples $\left\{X_{i}: i \leq\lfloor N \hat{\theta}\rfloor\right\}$ and $\left\{X_{i}: i>\lfloor N \hat{\theta}\rfloor\right\}$. Details are omitted for the sake of brevity.

Remark 3.2 The extension of the methodology to the analysis of multiple change point problems is of great practical interest and briefly indicated here. Let $0<\theta_{1}<\theta_{2}<\ldots<\theta_{K}<1$ denote the unknown change points and assume that

$$
X_{i}=\mu+\delta_{j-1}+f\left(\varepsilon_{i}, \varepsilon_{i-1}, \ldots\right) \quad \text { if } \quad\left\lfloor N \theta_{j-1}\right\rfloor+1 \leq i \leq\left\lfloor N \theta_{j}\right\rfloor ; \quad 1 \leq j \leq K+1
$$

where $\delta_{0}=0 ; \theta_{0}=0, \theta_{K+1}=1$. For the sake of simplicity we consider the same function $f$ as filter for the error process on $j$ 'th segment (in contrast to model (3.1)) and further consider $K$ as known. Defining the vector of integrated squared differences

$$
\Psi:=\left(\int_{T} \delta_{1}^{2}(t) d t, \int_{T}\left\{\delta_{2}(t)-\delta_{1}(t)\right\}^{2} d t, \ldots, \int_{T}\left\{\delta_{K}(t)-\delta_{K-1}(t)\right\}^{2} d t\right)^{\top},
$$

there are several possibilities to formulate relevant hypotheses in this setting. Here we will focus on

$$
H_{0}^{L^{2}}: \sum_{j=1}^{K} \Psi_{j} \leq \Delta
$$

which corresponds to the null that the sum of all integrated squared changes does not exceed a threshold $\Delta$ and

$$
H_{0}^{\infty}: \max _{j=1}^{K} \Psi_{j} \leq \Delta
$$

meaning that no single integrated squared change exceeds $\Delta$. Note that $H_{0}^{\infty}$ can be equivalently formulated as the intersection of the following hypotheses

$$
H_{0}^{(j)}: \Psi_{j} \leq \Delta \quad j=1, \ldots, K .
$$


For testing either of the hypotheses, we propose to first estimate the multiple change points by adapting one of the commonly used methods such as binary segmentation or wild binary segmentation [see for example Vostrikova (1981); Harchaoui and Lévy-Leduc (2010); Fryzlewicz (2014); Zhang and Lavitas (2018) among many others] to dependent functional data. The resulting estimator is denoted by $\hat{\theta}=\left(\hat{\theta}_{1}, \ldots, \hat{\theta}_{K}\right)$ and we put $\hat{\theta}_{K+1}=1, \hat{\theta}_{0}=0$. Next we consider generalizations of the statistics $\hat{\mathbb{D}}_{N}^{c p}$ and $\hat{\mathbb{V}}_{N}^{c p}$ in $(3.6)$ and $(3.7)$ on each segment

$$
S_{j}:=\left\{X_{i} \mid\left\lfloor N \hat{\theta}_{j-1}\right\rfloor+1 \leq i \leq\left\lfloor N \hat{\theta}_{j+1}\right\rfloor\right\} \quad(j=1, \ldots, K) .
$$

More precisely, define $\hat{N}_{j}:=\left\lfloor N \hat{\theta}_{j}\right\rfloor-\left\lfloor N \hat{\theta}_{j-1}\right\rfloor, j=1, \ldots, K+1$ as the sample size between the $(j-1)$ 'st and $j$ 'th estimated change point and let

$$
\hat{D}_{N, j}^{c p}(t, \lambda, \hat{\theta})=\frac{1}{\hat{N}_{j}} \sum_{i=1}^{\left\lfloor\lambda \hat{N}_{j}\right\rfloor} X_{\left\lfloor N \hat{\theta}_{j-1}\right\rfloor+i}(t)-\frac{1}{\hat{N}_{j+1}} \sum_{i=1}^{\left\lfloor\lambda \hat{N}_{j+1}\right\rfloor} X_{\left\lfloor N \hat{\theta}_{j}\right\rfloor+i}(t), \quad j=1, \ldots, K .
$$

Further, define

$$
\hat{\mathbb{D}}_{N, j}^{c p}(\lambda, \hat{\theta}):=\int_{T}\left(\hat{D}_{N, j}^{c p}(t, \lambda, \hat{\theta})\right)^{2} d t \quad(j=1, \ldots, K) .
$$

With this preparation, we first discuss a test for the hypothesis $H_{0}^{L^{2}}$ in 3.12 . Define

$$
\begin{aligned}
\hat{\mathbb{D}}_{N}^{L^{2}}(\lambda, \hat{\theta}) & :=\sum_{j=1}^{K} \hat{\mathbb{D}}_{N, j}^{c p}(\lambda, \hat{\theta}) \\
\hat{\mathbb{V}}_{N}^{L^{2}}(\hat{\theta}) & :=\left\{\int_{0}^{1}\left[\hat{\mathbb{D}}_{N}^{L^{2}}(\lambda, \hat{\theta})-\lambda^{2} \hat{\mathbb{D}}_{N}^{L^{2}}(1, \hat{\theta})\right]^{2} \nu(d \lambda)\right\}^{1 / 2} .
\end{aligned}
$$

We propose to reject $H_{0}^{L^{2}}$ whenever

$$
\hat{\mathbb{D}}_{N}^{L^{2}}(1, \hat{\theta})>\Delta+q_{1-\alpha}(\mathbb{W}) \hat{\mathbb{V}}_{N}^{L^{2}}(\hat{\theta})
$$

where $q_{1-\alpha}(\mathbb{W})$ denotes the $(1-\alpha)$ quantile of the random variable $\mathbb{W}$ defined in $(2.9)$. In Section B.3 of the Online Supplement, we will show that this provides a consistent and asymptotic level $\alpha$ test for $H_{0}^{L^{2}}$ under the following conditions:

(m1) The data are generated from model (3.11) with $f,\left\{\varepsilon_{i}\right\}_{i \in \mathbb{Z}}$ satisfying (A2)-(A4).

(m2) The true number of change points is $K$ (i.e. all entries of $\Psi$ are non-zero and there are no other change points) and $\hat{\theta}_{j}=\theta_{j}+o_{P}\left(N^{-1 / 2}\right),(j=1, \ldots, K)$.

Those assumptions are made for the sake of a simpler presentation. It is possible to generalize (m1) to the case where the filter $f$ changes in each segment as in model (3.1). Similarly, (m2) can be weakened to i.e. $\Psi_{j}=0$ for some values $j$. In this case the requirement $\hat{\theta}_{j}=\theta_{j}+o_{P}\left(N^{-1 / 2}\right)$ for $j$ with $\Phi_{j}=0$, is not realistic and has to be replaced by a different condition. Details are 
omitted for the sake of brevity.

Finally, we briefly comment on testing the hypothesis (3.13) using self-normalization which is a substantially more challenging problem. Although it is possible to construct self-normalized test statistics for each of the hypotheses $H_{0}^{(j)}$ in 3.14 separately, note that neighbouring segments $S_{j}, S_{j+1}$ overlap so that in the limit those self-normalized statistics become dependent. It can further be shown that, although the marginal distributions are pivotal, the resulting joint distribution is not pivotal any more. Therefore one option to construct a test for the hypothesis 3.13 is to apply a multiple testing correction after testing each $H_{0}^{(j)}$ separately based on self-normalization. Problems of this type have been discussed more intensively the context of testing simultaneously several hypotheses of equivalence of the form (1.3) [see Munk and Pflüger (1999); Wang et al. (1999)]. These references indicate the general difficulty to construct tests for multiple precise hypotheses using the joint distribution of a vector of test statistics, and we leave a detailed investigation of this problem for future research.

\section{$4 \quad$ Inference for covariance operators}

In this section we briefly discuss extensions of the methodology in Sections 2 and 3 to test similar hypotheses regarding the covariance operators of functional time series. For the sake of brevity we omit the one sample case and focus on the two sample case and the change point setting. The problem of testing the classical hypothesis has found considerable attention in the literature and we refer to Panaretos et al. (2010), Fremdt et al. (2013), Guo et al. (2016) and Paparoditis and Sapatinas (2016), who developed methodology for comparing covariance operators for independent functional data. Pilavakis et al. (2019) proposed a test for the equality

of the lag-0 auto-covariance operators of $K$ functional time series, and Sharipov and Wendler (2019) considered bootstrap-based statistical inference for covariance operators of functional time series. Change point analysis for covariance operators was developed by Aston and Kirch (2012b) and Stoehr et al. (2019), among others, while Zhang and Shao (2015) and Aue et al. (2018) discussed statistical inference tools for the eigen-system of covariance operators.

We will repeatedly make use of the following strengthening of Assumptions (A3)-(A4) on a sequence of $L^{2}(T)$-valued random elements $\left\{\eta_{j}\right\}_{j \in \mathbb{Z}}$ that satisfy (A2):

(A3') $\mathbb{E}\left\|\eta_{j}\right\|^{4+\psi}<\infty$ for some $\psi \in(0,1)$.

(A4') The sequence $\left(\eta_{j}\right)_{j \in \mathbb{Z}}$ can be approximated by $\ell$-dependent sequences $\left(\eta_{j, \ell}\right)_{j \in \mathbb{Z}}$ in the sense that for some $\kappa>4+\psi$

$$
\sum_{\ell=1}^{\infty}\left(\mathbb{E}\left\|\eta_{0}-\eta_{0, \ell}\right\|^{4+\psi}\right)^{1 / \kappa}<\infty
$$

where $\eta_{j, \ell}$ is defined by

$$
\begin{aligned}
& \eta_{j, \ell}=f\left(\varepsilon_{j}, \varepsilon_{j-1}, \ldots \varepsilon_{j-\ell+1}, \boldsymbol{\varepsilon}_{j, \ell}^{*}\right) \\
& \varepsilon_{j, \ell}^{*}=\left(\varepsilon_{j, \ell, j-\ell}^{*}, \varepsilon_{j, \ell, j-\ell-1}^{*}, \ldots\right)
\end{aligned}
$$


and the random variables $\varepsilon_{j, \ell, k}^{*}$ are i.i.d. copies of $\varepsilon_{0}$, and independent of the sequence $\left(\varepsilon_{j}\right)_{j \in \mathbb{Z} \cdot}$

\subsection{Two sample problem}

Given two samples $X_{1}, \ldots, X_{m}$ and $Y_{1}, \ldots, Y_{n}$ from independent, strictly stationary functional time series $\left\{X_{t}\right\}_{t \in \mathbb{Z}},\left\{Y_{t}\right\}_{t \in \mathbb{Z}}$, we are interested in testing the null hypothesis of no relevant difference in the covariance operators $C^{X}, C^{Y}$ where

$$
C^{X}(s, t):=\mathbb{E}\left[\left(X_{0}(s)-\mathbb{E}\left[X_{0}(s)\right]\right)\left(X_{0}(t)-\mathbb{E}\left[X_{0}(t)\right]\right)\right]
$$

and $C^{Y}$ is defined similarly. Thus we investigate the hypotheses

$$
H_{0}^{C}: d_{C}=\int_{T} \int_{T} D_{C}^{2}(s, t) d s d t \leq \Delta \quad \text { versus } \quad H_{1}^{C}: d_{C}>\Delta
$$

where $D_{C}(s, t)=C^{X}(s, t)-C^{Y}(s, t)$ denotes the difference of the covariance operators at the points $s, t \in T$. We reject the null hypothesis in (4.1), whenever

$$
\hat{\mathbb{D}}_{m, n}^{C}>\Delta+q_{1-\alpha}(\mathbb{W}) \hat{\mathbb{V}}_{m, n}
$$

where $q_{1-\alpha}(\mathbb{W})$ denotes the $(1-\alpha)$-quantile of the random variable $\mathbb{W}$ defined in $(2.9)$,

$$
\begin{aligned}
\hat{\mathbb{D}}_{m, n}^{C} & =\int_{T} \int_{T} D_{m, n}^{2}(s, t, 1) d s d t \\
\hat{\mathbb{V}}_{m, n}^{C} & =\left(\int_{0}^{1}\left[\int_{T} \int_{T} D_{m, n}^{2}(s, t, \lambda) d s d t-\lambda^{2} \int_{T} \int_{T} D_{m, n}^{2}(s, t, 1) d s d t\right]^{2} \nu(d \lambda)\right)^{1 / 2}
\end{aligned}
$$

and $D_{m, n}$ is the partial sum defined by

$$
\begin{aligned}
D_{m, n}(s, t, \lambda)= & \frac{1}{m-1} \sum_{j=1}^{\lfloor m \lambda\rfloor}\left(X_{j}(s)-\frac{1}{\lfloor m \lambda\rfloor \vee 1} \sum_{i=1}^{\lfloor m \lambda\rfloor} X_{i}(s)\right)\left(X_{j}(t)-\frac{1}{\lfloor m \lambda\rfloor \vee 1} \sum_{i=1}^{\lfloor m \lambda\rfloor} X_{i}(t)\right) \\
& -\frac{1}{n-1} \sum_{j=1}^{\lfloor n \lambda\rfloor}\left(Y_{j}(s)-\frac{1}{\lfloor n \lambda\rfloor \vee 1} \sum_{i=1}^{\lfloor n \lambda\rfloor} Y_{i}(s)\right)\left(Y_{j}(t)-\frac{1}{\lfloor n \lambda\rfloor \vee 1} \sum_{i=1}^{\lfloor n \lambda\rfloor} Y_{i}(t)\right) .
\end{aligned}
$$

The following result shows that the decision rule in 4.2 defines a consistent asymptotic level $\alpha$-test for the hypotheses $H_{0}^{C}$ versus $H_{1}^{C}$ provided that $\Delta>0$. For a precise statement, define

$$
\tau_{D_{C}}^{2}=4 \int_{T} \int_{T} \int_{T} \int_{T} D_{C}(s, t) D_{C}\left(s^{\prime}, t^{\prime}\right)\left(\frac{1}{\rho} C_{X}\left((s, t),\left(s^{\prime}, t^{\prime}\right)\right)+\frac{1}{1-\rho} C_{Y}\left((s, t),\left(s^{\prime}, t^{\prime}\right)\right)\right) d s d t d s^{\prime} d t^{\prime}
$$

where $\rho$ comes from assumption (B1) and $C_{X}$ and $C_{Y}$ denote the long-run covariance kernels corresponding to the time series $\left\{X_{t} \otimes X_{t}\right\}_{t \in \mathbb{Z}}$ and $\left\{Y_{t} \otimes Y_{t}\right\}_{t \in \mathbb{Z}}$, respectively. 
Theorem 4.1 Assume that $\Delta>0$. Let assumption (B1) from Section 2.2 hold and assume that the functional time series $\left\{X_{t}\right\}_{t \in \mathbb{Z}},\left\{Y_{t}\right\}_{t \in \mathbb{Z}}$ satisfy (A1) with means $\mu_{X}, \mu_{Y}$ and errors $\eta_{j}^{X}, \eta_{j}^{Y}$, (A2), and that $\eta_{j}^{X}, \eta_{j}^{Y}$ satisfy (A3'), (A4'). Finally, assume that $\nu$ puts no mass in a neighbourhood of zero. Then

$$
\lim _{n \rightarrow \infty} \mathbb{P}\left(\hat{\mathbb{D}}_{m, n}^{C}>\Delta+q_{1-\alpha}(\mathbb{W}) \mathbb{V}_{n, m}^{C}\right)= \begin{cases}0 & \text { if } d_{C}<\Delta, \\ \alpha & \text { if } d_{C}=\Delta \text { and } \tau_{D_{C}}^{2}>0 \\ 1 & \text { if } d_{C}>\Delta .\end{cases}
$$

Similarly to the setting of testing for a relevant difference in the means it can be shown that the rejection rule in 4.2 does not lead to an asymptotic level $\alpha$ test when $\Delta=0$.

\subsection{Change point problem}

For an extension of the methodology to testing for relevant changes in the covariance structure of a time series, we will assume that data are generated from the model

$$
X_{i}= \begin{cases}\mu+f_{1}\left(\varepsilon_{i}, \varepsilon_{i-1, \ldots}\right) & \text { if } i \leq N \theta_{0} \\ \mu+f_{2}\left(\varepsilon_{i}, \varepsilon_{i-1, \ldots}\right) & \text { if } i>N \theta_{0}\end{cases}
$$

Define $\eta_{i}^{(k)}:=f_{k}\left(\varepsilon_{i}, \varepsilon_{i-1, \ldots}\right), k=1,2$ and denote by $C_{1}, C_{2}$ the covariance operators of the process $X_{i}$ before and after the structural break. We are now interested in testing the hypotheses

$$
H_{0}^{C}: d_{C}^{c p}=\int_{T} \int_{T}\left\{C_{1}(s, t)-C_{2}(s, t)\right\}^{2} d s d t \leq \Delta \quad \text { versus } \quad H_{1}^{C}: d_{C}^{c p}>\Delta .
$$

Similarly to Section 3 we first construct an estimator for the unknown change point location $\theta_{0}$. To this end we define

$$
\bar{X}_{\ell: k}(t):=\frac{1}{k-\ell+1} \sum_{i=\ell}^{k} X_{i}(t)
$$

and consider the covariance estimators

$$
\begin{aligned}
\hat{C}_{1: k}(s, t) & :=\frac{1}{k-1} \sum_{j=1}^{k}\left(X_{j}(s)-\bar{X}_{1: k}(s)\right)\left(X_{j}(t)-\bar{X}_{1: k}(t)\right) \\
\hat{C}_{k+1: N}(s, t) & :=\frac{1}{N-k-1} \sum_{j=k+1}^{N}\left(X_{j}(s)-\bar{X}_{k+1: N}(s)\right)\left(X_{j}(t)-\bar{X}_{k+1: N}(t)\right)
\end{aligned}
$$

for $k=2, \ldots, N-2$. Next fix a trimming parameter $\varepsilon \in[0,1 / 2)$ and define the estimator

$$
\hat{\theta}^{C o v}:=\frac{1}{N} \operatorname{argmax}_{\lfloor N \varepsilon\rfloor+1 \leq k \leq N-\lfloor N \varepsilon\rfloor} \hat{f}^{C o v}(k),
$$


where $\hat{f}^{C o v}(0)=\hat{f}^{C o v}(N)=0$ and for $k=1, \ldots, N-1$

$$
\hat{f}^{C o v}(k):=\frac{k}{N}\left(1-\frac{k}{N}\right) \int_{T} \int_{T}\left(\hat{C}_{1: k}(s, t)-\hat{C}_{k+1: N}(s, t)\right)^{2} d s d t .
$$

Following the approach in Section 3 , for arbitrary $\theta \in[2 / N, 1-1 / N)$ define

$$
\begin{array}{r}
D_{N}^{c p, C o v}(s, t, \lambda, \theta)=\frac{1}{\lfloor N \theta\rfloor-1} \sum_{j=1}^{\lfloor\lfloor\theta\rfloor \lambda\rfloor}\left(X_{j}(s)-\bar{X}_{1:\lfloor\lfloor N \theta\rfloor \lambda\rfloor}(s)\right)\left(X_{j}(t)-\bar{X}_{1:\lfloor\lfloor\theta\rfloor \lambda\rfloor}(t)\right) \\
-\frac{1}{N-\lfloor N \theta\rfloor-1} \sum_{j=\lfloor\theta N\rfloor+1}^{\lfloor\theta N\rfloor+\lfloor\lambda(N-\lfloor\theta N\rfloor)\rfloor}\left(X_{j}(s)-\bar{X}_{\lfloor N \theta\rfloor+1:(\lfloor N \theta\rfloor+\lfloor\lambda(N-\lfloor N \theta\rfloor)}(s)\right) \\
\times\left(X_{j}(t)-\bar{X}_{\lfloor N \theta\rfloor+1:(\lfloor N \theta\rfloor+\lfloor\lambda(N-\lfloor N \theta\rfloor)}(t)\right)
\end{array}
$$

and consider the statistics

$$
\begin{aligned}
\hat{\mathbb{D}}_{N}^{c p, C o v} & =\int_{T} \int_{T}\left(D_{N}^{c p, C o v}(s, t, 1, \hat{\theta})\right)^{2} d s d t \\
\hat{\mathbb{V}}_{N}^{c p, C o v} & =\left(\int_{0}^{1}\left[\int_{T} \int_{T}\left(D_{N}^{c p, C o v}(s, t, \lambda, \hat{\theta})\right)^{2} d t-\lambda^{2} \int_{T} \int_{T}\left(D_{N}^{c p, C o v}(s, t, 1, \hat{\theta})^{2} d s d t\right)\right]^{2} \nu(d \lambda)\right)^{1 / 2},
\end{aligned}
$$

where $\nu$ is a probability measure on the interval $(0,1)$. The test for the hypotheses (4.4) rejects $H_{0}^{C}$, whenever

$$
\hat{\mathbb{D}}_{N}^{c p, C o v}>\Delta+q_{1-\alpha}(\mathbb{W}) \hat{\mathbb{V}}_{N}^{c p, C o v}
$$

where $q_{1-\alpha}(\mathbb{W})$ is the $(1-\alpha)$-quantile of the distribution of the random variable $\mathbb{W}$ in $(2.9)$.

For a precise statement of the next theorem, we define

$$
\tau_{\delta_{C}, \theta_{0}}^{2}=4 \int_{T} \int_{T} \int_{T} \int_{T} \delta_{C}(s, t) \delta_{C}\left(s^{\prime}, t^{\prime}\right)\left(\frac{1}{\theta_{0}} K_{1}\left((s, t),\left(s^{\prime}, t^{\prime}\right)\right)+\frac{1}{1-\theta_{0}} K_{2}\left((s, t),\left(s^{\prime}, t^{\prime}\right)\right)\right) d s d t d s^{\prime} d t^{\prime},
$$

where $\delta_{C}(s, t)=C_{2}(s, t)-C_{1}(s, t)$ and $K_{j}$ is the long-run covariance kernel of $\left\{\eta_{i}^{(j)} \otimes \eta_{i}^{(j)}\right\}_{i \in \mathbb{Z}}$.

Theorem 4.2 Assume that the data is generated according to model 4.3 , $\theta_{0} \in(\varepsilon, 1-\varepsilon)$, and that $\left(\varepsilon_{j}\right)_{j \in \mathbb{Z}}$ together with $f_{1}, f_{2}$ satisfy (A2), (A3'), (A4'). Further assume that $\nu$ puts no mass in a neighbourhood of zero. Then the decision rule in (4.6) satisfies

$$
\lim _{n \rightarrow \infty} \mathbb{P}\left(\hat{\mathbb{D}}_{N}^{c p, C o v}>\Delta+q_{1-\alpha}(\mathbb{W}) \hat{\mathbb{V}}_{N}^{c p, C o v}\right)= \begin{cases}0 & \text { if } d_{C}^{c p}<\Delta \\ \alpha & \text { if } d_{C}^{c p}=\Delta \text { and } \tau_{\delta_{C}, \theta_{0}}^{2}>0 \\ 1 & \text { if } d_{C}^{c p}>\Delta .\end{cases}
$$

Similarly to the setting of testing for a relevant change-point in the mean it can be shown that the rejection rule in 4.6 does not lead to an asymptotic level $\alpha$ test when $\Delta=0$. 


\section{$5 \quad$ Finite sample properties}

In this section we illustrate the finite sample properties of the new procedures by means of a simulation study. Note that one has to specify the measure $\nu$ used in the definition of the normalizer (2.6), 2.19) and (3.7) and we use $\nu=\frac{1}{19} \sum_{i=1}^{19} \delta_{i / 20}$ throughout this section if not mentioned otherwise; here $\delta_{\lambda}$ denotes the Dirac measure at the point $\lambda \in[0,1]$. For example, for this choice the quantity $\hat{\mathbb{V}}_{n}$ defined in 2.6 is given by

$$
\hat{\mathbb{V}}_{n}=\left[\frac{1}{19} \sum_{i=1}^{19}\left(\int_{T} S_{n}^{2}\left(t, \frac{i}{20}\right) d t-\left(\frac{i}{20}\right)^{2} \int_{T} S_{n}^{2}(t, 1) d t\right)^{2}\right]^{1 / 2}
$$

and the other expressions are obtained similarly. In the following sections we discuss the one sample case, the two sample case and change point detection separately. All results are based on 1000 simulation runs.

\subsection{One sample problems}

We consider a process $\left\{X_{n}\right\}_{n \in \mathbb{N}}$ with expectation function

$$
\mu(t)=\sqrt{2 \delta} \sin (2 \pi t)
$$

and different error processes, where we investigate a similar scenario as Aue et al. (2015) (see Sections 6.3 and 6.4 in the latter paper). More precisely, let $X_{n}=\mu+\varepsilon_{n}$ and consider $B$-spline basis functions $b_{1}, \ldots, b_{D}(D \in \mathbb{N})$. Define the linear space $\mathbb{H}=\operatorname{span}\left\{b_{1}, \ldots, b_{D}\right\} \subset L^{2}([0,1])$ and independent processes $\eta_{1}, \ldots, \eta_{n} \in \mathbb{H}$ by

$$
\eta_{j}=\sum_{i=1}^{D} N_{i, j} b_{i} \quad(j=1, \ldots, n),
$$

where $N_{1,1}, N_{2,1}, \ldots, N_{D, n}$ are independent $N\left(0, \sigma_{i}^{2}\right)(i=1, \ldots, D ; j=1, \ldots, n)$ distributed random variables. Our first example considers independent error processes of the form

$$
\varepsilon_{j}=\eta_{j} \quad(j \in \mathbb{Z})
$$

while the second example investigates a functional moving average $f M A(1)$ process given by

$$
\varepsilon_{j}=\eta_{j}+\Theta \eta_{j-1} \quad(j \in \mathbb{Z}) .
$$

Here the operator $\Theta: \mathbb{H} \rightarrow \mathbb{H}$ (acting on finite dimensional spaces) is defined by the matrix $\Theta=\left(\Theta_{i j}\right)_{i, j=1}^{D} \in \mathbb{R}^{D \times D}$, where the entries $\Theta_{i j}$ are normally distributed with mean zero and standard deviation $\kappa \sigma_{i} \sigma_{j}$ and $\kappa$ is a scaling factor such that the resulting matrix $\Theta$ has (induced) spectral norm equal to 0.7 . The operator $\Theta$ is newly generated in every simulation run (see Sections 6.3 and 6.4 in Aue et al. (2015) for a similar approach) and we use $D=21$. The third error structure under consideration are independent Brownian Bridges. 
In Figure 1 we display the simulated rejection probabilities of the test 2.8 for the hypotheses (2.1), where $\Delta=0.02$, which corresponds to the value $\delta=0.02$ in model (5.1). These results show a pattern which is in line with the theoretical findings in Theorem 2.1. For example at the boundary of the null hypotheses, i.e. for $\delta=\Delta=0.02$, the simulated level is close to the nominal level. In the interior of the null hypothesis $(\delta<\Delta)$ the simulated rejection probabilities are strictly smaller than $\alpha=0.05$, while they are strictly larger than 0.05 in the interior of the alternative, i.e. $\delta>\Delta$.
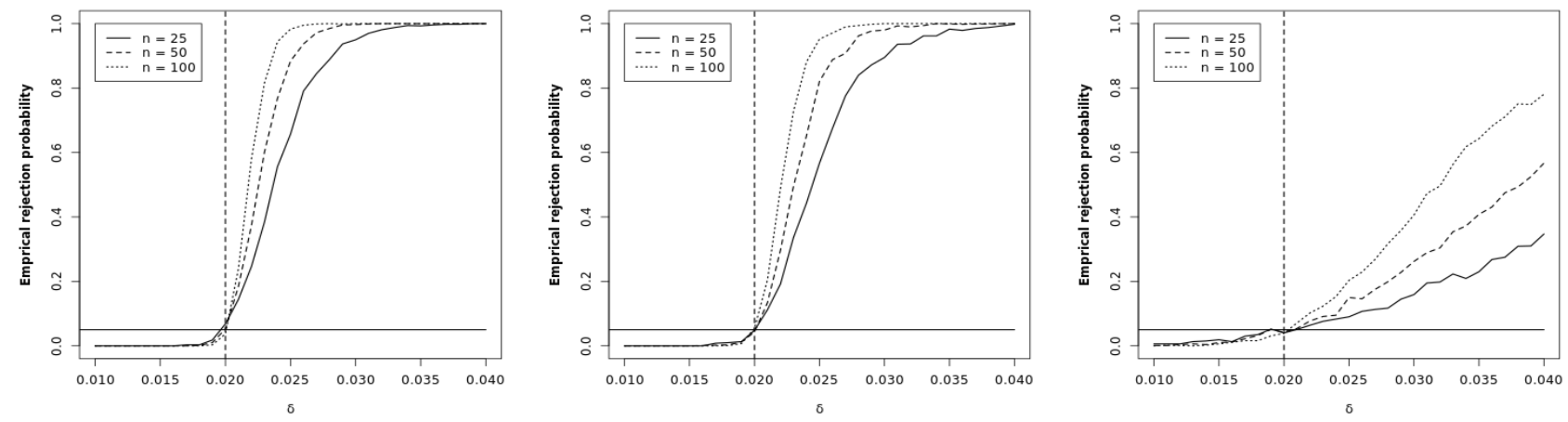

Figure 1: Simulated rejection probabilities of the test (2.8) for the relevant hypotheses (2.1) with $\Delta=0.02$. The mean function is given by (5.1) and different error processes are considered. Left panel: independent error processes defined by (5.3). Middle panel: $f M A(1)$ processes defined by (5.4). Right panel: independent Brownian Bridges.

\subsubsection{Estimating the long-run variance}

It is of interest to compare the procedure based on self-normalization with the test (2.13) defined in Remark 2.3, which uses an estimate of the long-run variance. For this comparison, we also implement the (practically infeasible) test which rejects the null hypothesis of no relevant difference whenever

$$
\hat{\mathbb{T}}_{n}>\Delta+u_{1-\alpha} \frac{\tau}{\sqrt{n}}
$$

that is we use the true asymptotic standard deviation $\tau$ instead of its estimate $\hat{\tau}_{n}$. Throughout this section we consider fAR(1) error processes defined by

$$
\varepsilon_{j}=\eta_{j}+\kappa \varepsilon_{j-1} \quad(j \in \mathbb{Z})
$$

for some $\kappa \in(0,1)$ and expectation function $\mu$ as in (5.1). The random functions $\eta_{j}$, for $j=$ $1, \ldots, n$, are defined as in 5.2 (again with $D=21$ ) but in this section, we use the Fourier functions defined by $b_{1} \equiv 1$ and

$$
b_{j}(t)= \begin{cases}\sqrt{2} \sin (j \pi t), & j \text { is even } \\ \sqrt{2} \cos ((j-1) \pi t), & j>1 \text { is odd }\end{cases}
$$


as (orthonormal) basis functions such that an explicit calculation of the long-run variance becomes easier. Thus we have $\operatorname{Cov}\left(\varepsilon_{0}(s), \varepsilon_{\ell}(t)\right)=\operatorname{Cov}\left(\eta_{0}(s), \eta_{0}(t)\right) \kappa^{\ell} /(1-\kappa)^{2}$ which yields

$$
\tau^{2}=4 \int_{T} \int_{T} \mu(s) \mu(t) C(s, t) d s d t=\frac{4}{(1-\kappa)^{2}} \sum_{i=1}^{D} \frac{1}{i^{2}}\left(\int_{0}^{1} \mu(t) b_{i}(t) d t\right)^{2} .
$$

In order to obtain an estimate of the long-run covariance function $C$, we use the "opt_bandwidth" function from the R package "fChange" with the Bartlett kernel (both as "kern_type" and as " $\left.k e r n \_t y p e \_i n i "\right)$.
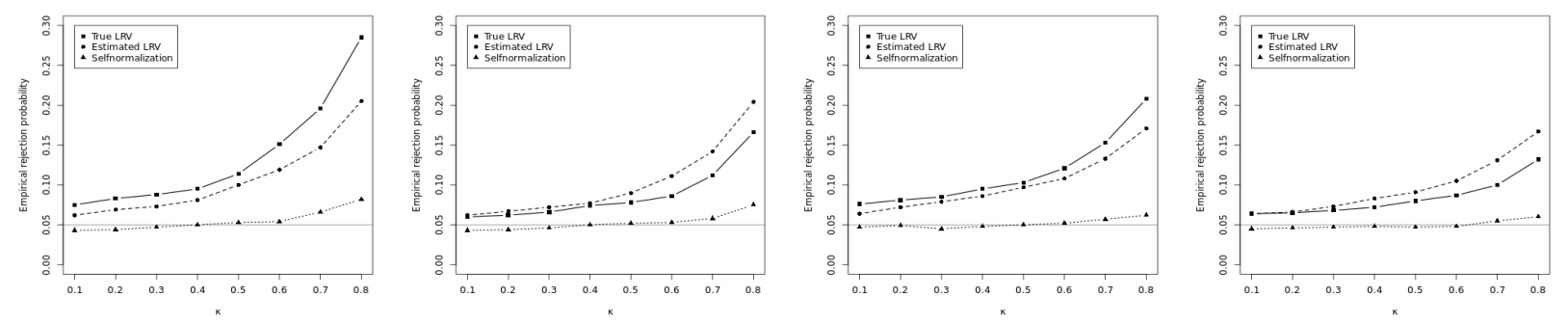

Figure 2: Approximation of the test level for different values of $\kappa$. Errors are $f A R(1)$ processes defined by (5.6). Left to right: $\Delta=0.5, n=100 ; \Delta=1.5, n=100 ; \Delta=0.5, n=200$; $\Delta=1.5, n=200$.

In Figure 2, we compare the approximation of the nominal level of the three tests for different values of $n, \kappa, \Delta$ at the boundary of the null hypothesis, that is for $\int_{T} \mu^{2}(t) d t=\Delta$. We observe that the self-normalized test performs well across all settings considered with only a slight inflation of level for the most difficult case $\kappa=0.8, n=100$. In contrast, even for a large sample size $n=200$, the tests based on the estimated and true (asymptotic) long-run variance exceed their nominal level for all values of $\kappa$ considered with especially large over-rejections for $\kappa>0.5$. Interestingly, the test based on the estimated long-run variance performs slightly better compared to the test with the true asymptotic long-run variance when $n=100$. A similar pattern can be observed for data that are more heavy-tailed. Due to space considerations additional details are deferred to Section A.5 in the online supplement.

\subsection{Two sample problem}

We begin considering the case of two independent (stationary) samples, $X_{1}, \ldots, X_{m}$ and $Y_{1}, \ldots, Y_{n}$, with $\mathbb{E}\left[X_{i}\right]=\mu_{1}$ and $\mathbb{E}\left[Y_{j}\right]=\mu_{2}$, where the mean functions are given by

$$
\mu_{1} \equiv 0, \quad \mu_{2}(t)=a t(1-t)
$$

[see Section 4 in Horváth et al. (2013) for a similar approach], such that $\int_{0}^{1} \mu_{2}^{2}(t) d t=a^{2} / 30$. We are interested in testing the hypotheses (2.16), that is

$$
H_{0}: \int_{T} D^{2}(t) d t \leq \Delta \quad \text { versus } \quad H_{1}: \int_{T} D^{2}(t) d t>\Delta,
$$


where $D=\mu_{1}-\mu_{2}$ is the (unknown) difference of the two mean functions and the threshold is given by $\Delta=0.2^{2} / 30$ (note that this corresponds to the choice $a=0.2$ ). We consider independent samples, $f M A(1)$ processes (generated as described in Section 5.1) and independent Brownian Bridges as error processes.
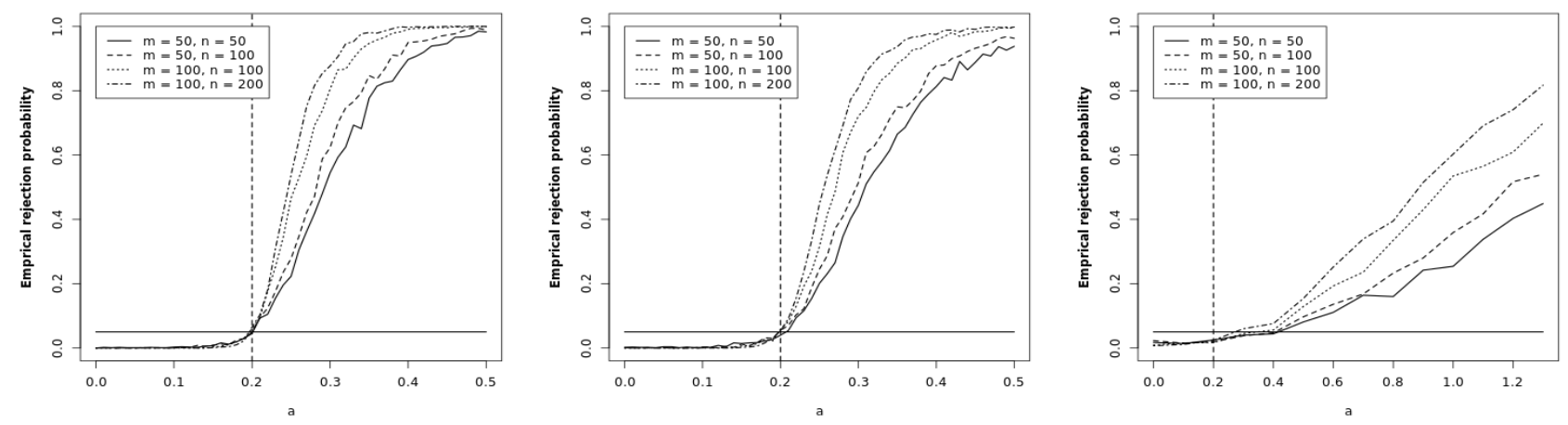

Figure 3: Simulated rejection probabilities of the test (2.17) for the relevant hypotheses (2.16) with $\Delta=0.2^{2} / 30$. The mean functions are given by (5.8) and different independent error processes are considered. First panel: independent error processes defined by (5.3). Second panel: $f M A(1)$ processes defined by (5.4). Third panel: Brownian Bridges.

In Figure 3 we display the rejection probabilities of the test 2.17) as a function of the parameter $a$ for different sample sizes $m$ and $n$. We observe that the test yields a good approximation of the nominal level at the boundary $a=0.2^{2} / 30$ and detects the alternatives with reasonable power. Further results for dependent samples are presented in Section A.2 of the online supplement and show a similar picture.

We conclude this section investigating the effect of more heavy-tailed data and compare the test (2.17) with the two tests obtained by the alternative self-normalizations in (2.20) and (2.21). To be precise these tests reject the null hypothesis of no relevant difference, whenever

$$
\begin{aligned}
& \hat{\mathbb{D}}_{m, n}>\Delta+q_{1-\alpha}\left(\mathbb{W}^{\star}\right) \hat{\mathbb{V}}_{m, n}^{\star}, \\
& \hat{\mathbb{D}}_{m, n}>\Delta+q_{1-\alpha}\left(\mathbb{W}^{\star \star}\right) \hat{\mathbb{V}}_{m, n}^{\star \star},
\end{aligned}
$$

where $q_{1-\alpha}\left(\mathbb{W}^{\star}\right)$ and $q_{1-\alpha}\left(\mathbb{W}^{\star \star}\right)$ are the $(1-\alpha)$-quantiles of $\mathbb{W}^{\star}$ and $\mathbb{W}^{\star \star}$ in $(2.22)$.

In the left panel of Figure 4, we display the rejection probabilities of the tests (2.17), (5.9) and (5.10) in the situation considered in Figure 3. More precisely, the sample sizes are $m=50$, $n=100$, the mean functions are given by (5.8) and the error process is an $f M A(1)$ process defined by (5.4). We observe a very similar behaviour of all three tests under consideration.

Next we investigate a similar situation for more heavy-tailed data and consider similar error 

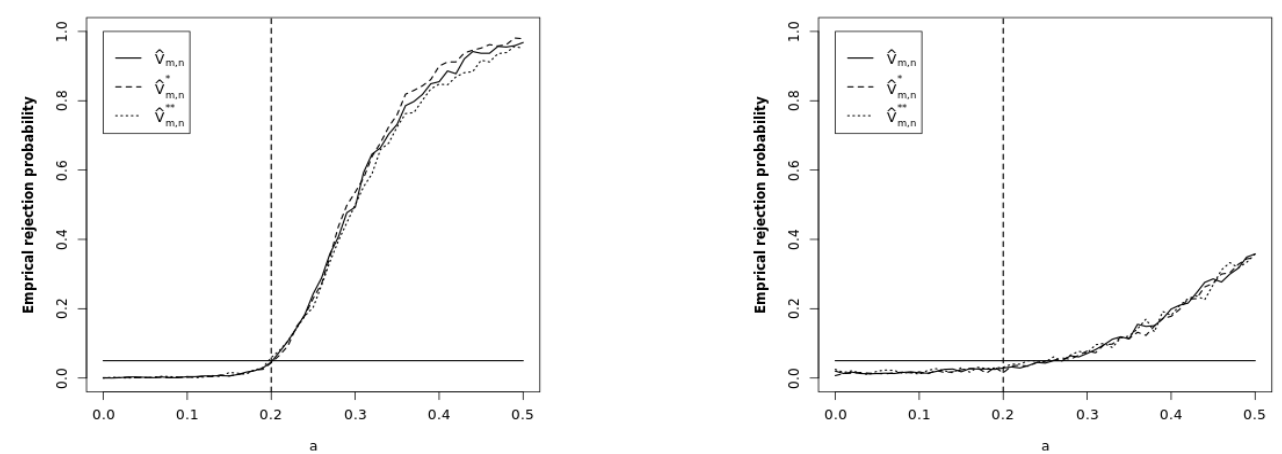

Figure 4: Simulated rejection probabilities of the tests (2.17), (5.9) and (5.10) using different self-normalizing factors. The mean functions are given by (5.8) and different independent error processes are considered with sample sizes $m=50, n=100$. The threshold is defined as $\Delta=$ $0.2^{2} / 30$ and the errors are $f M A(1)$ processes given by (5.4) (left panel) and (5.11) (right panel).

processes as used in Kraus and Panaretos (2012), that is

$$
\begin{aligned}
& \eta_{i}(t)=\frac{1}{\sqrt{10}} \sum_{k=1}^{10}\left\{k^{-3 / 2} \sqrt{2} \sin (2 \pi k t) V_{i, k}+3^{-k / 2} \sqrt{2} \cos (2 \pi k t) W_{i, k}\right\} \\
& \tilde{\eta}_{j}(t)=\frac{1}{\sqrt{10}} \sum_{k=1}^{10}\left\{k^{-3 / 2} \sqrt{2} \sin (2 \pi k t) \tilde{V}_{j, k}+3^{-k / 2} \sqrt{2} \cos (2 \pi k t) \tilde{W}_{j, k}\right\}
\end{aligned}
$$

$(i=1, \ldots, m, j=1, \ldots, n)$. Here the random variables $V_{i, k}, W_{i, k}, \tilde{V}_{j, k}, \tilde{W}_{j, k}$ are independent $t_{5^{-}}$ distributed random variables scaled to have unit variance. The right panel of Figure 4 shows the empirical rejection probabilities. We observe a very similar behaviour of the three considered tests. Additional results with b-spline basis functions show a similar picture and details are deferred to Section A.1 in the online supplement.
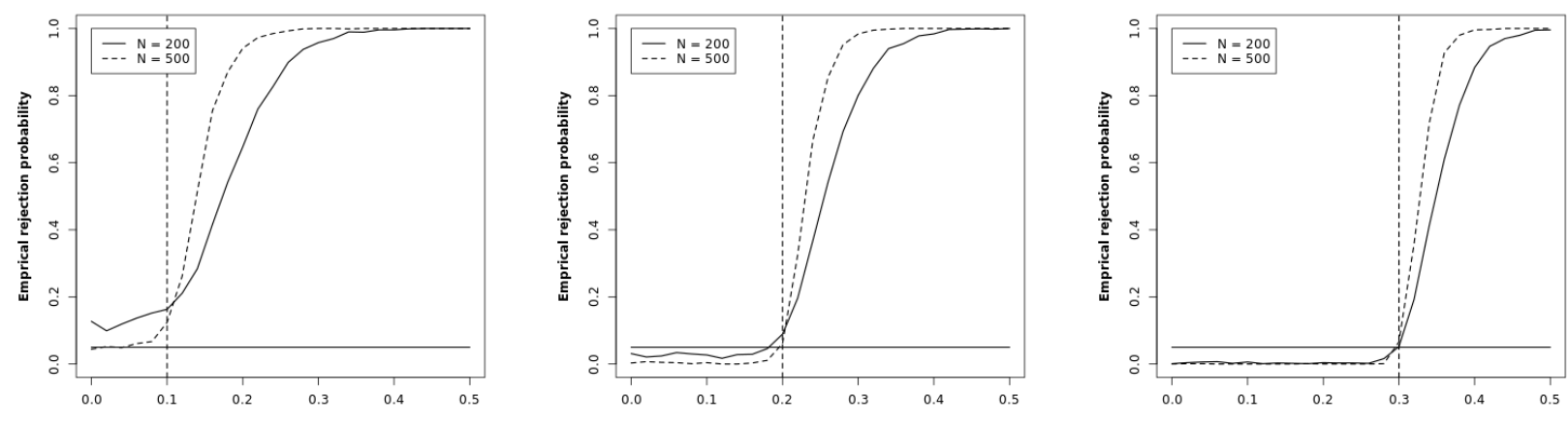

Figure 5: Simulated rejection probabilities of the test (3.8) for the relevant hypotheses (3.2) with $\Delta=0.1^{2} / 30$ (left), $\Delta=0.2^{2} / 30$ (middle), $\Delta=0.3^{2} / 30$ (right). Data is generated according to model (3.1) with $\theta_{0}=0.5, \mu=0, \delta(t)=$ at $(1-t)$, for $a=0,0.02, \ldots, 0.5$, and the errors are i.i.d. defined by (5.3). The tuning parameter is set to $\varepsilon=0.05$. 


\subsection{Change point problem}

We begin considering the model (3.1) with $\theta_{0}=0.5, \mu=0, \delta(t)=a t(1-t)$, the errors are i.i.d. from (5.3). The trimming parameter $\varepsilon$ for estimating the change point location is set to 0.05. Data are generated with $a=0,0.02, \ldots, 0.5$ and then empirical rejection probabilities are calculated using $\Delta=0.1^{2} / 30,0.2^{2} / 30,0.3^{2} / 30$. These probabilities are shown in Figure 5 .

From Theorem 3.1, we expect that the probability of rejection should be close to $\alpha$ at the boundary of the hypotheses $\left(\int_{0}^{1} D^{2}(t) d t=\Delta\right)$, strictly smaller than $\alpha$ in the interior of the null hypothesis $\left(\int_{0}^{1} D^{2}(t) d t<\Delta\right)$ and larger than $\alpha$ in the interior of the alternative $\left(\int_{0}^{1} D^{2}(t) d t>\Delta\right)$. This pattern is clearly observed for relevant hypotheses with threshold $\Delta \geq 0.2^{2} / 30$. However the proposed test is oversized if relevant hypotheses with $\Delta=0.1^{2} / 30$ are tested (see the left panel in Figure 5). This is because change point tests for relevant hypotheses require a precise estimate of the change point For small values of $a$ it is extremely difficult to estimate the true change point location, and an imprecise estimation of the change point results in a less accurate approximation of the nominal level. The difficulty of estimating the true change point location for small values of $a$ is further illustrated in Figure 6 where we show the histogram of the corresponding estimator of the change point for $a=0.1,0.2,0.3$ with sample size $N=200$.

Next, we investigate the properties of our test with dependent error processes, i.e we generate a $f M A(1)$ process $\left\{\eta_{i}\right\}_{i \in \mathbb{Z}}$ as described in Section 5.1 and define

$$
\begin{gathered}
X_{i}=\mu+\eta_{i}, \quad i=1, \ldots,\left\lfloor\theta_{0} N\right\rfloor ; \quad X_{i}=\mu+\delta+\eta_{i}, \quad i=\left\lfloor\theta_{0} N\right\rfloor+1, \ldots, N \\
X_{i}=\mu+\eta_{i}, \quad i=1, \ldots,\left\lfloor\theta_{0} N\right\rfloor ; \quad X_{i}=\mu+\delta+\sqrt{3} \eta_{i}, \quad i=\left\lfloor\theta_{0} N\right\rfloor+1, \ldots, N
\end{gathered}
$$

as the first and second scenario. The functions $\mu, \delta$ are as described in the beginning of this section. The corresponding rejection probabilities of the test (3.8) are depicted in Figure 7 where we restrict our attention to the case $\Delta=0.3^{2} / 30$ for the sake of brevity. We find that for both error settings the test performs reasonably well.
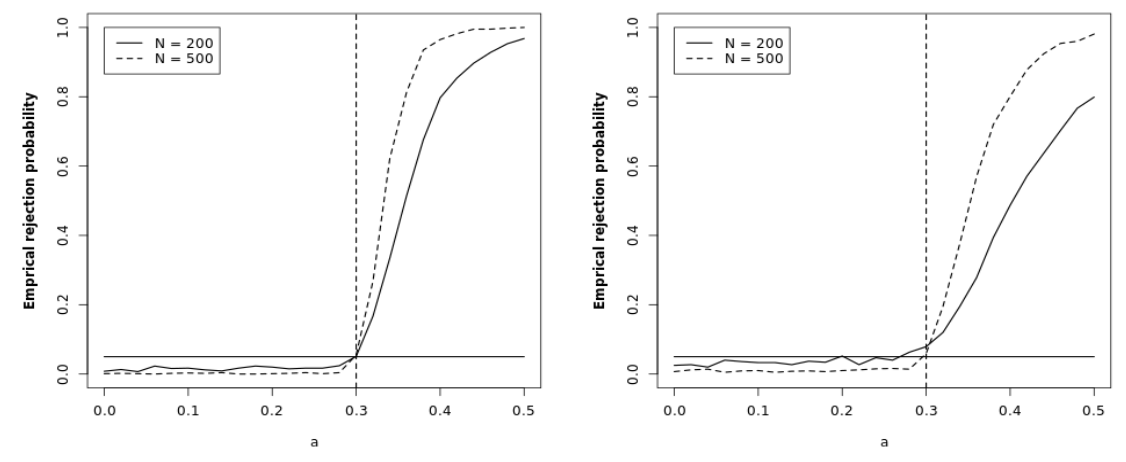

Figure 7: Simulated rejection probabilities of the test (3.8) for the relevant hypotheses (3.2) with $\Delta=0.3^{2} / 30$ in the case of $f M A(1)$ samples. The mean function after the change point is given by (5.8) and the mean function before the change point is the zero function. Left panel: error processes defined by (5.12). Right panel: error processes defined by (5.13). 

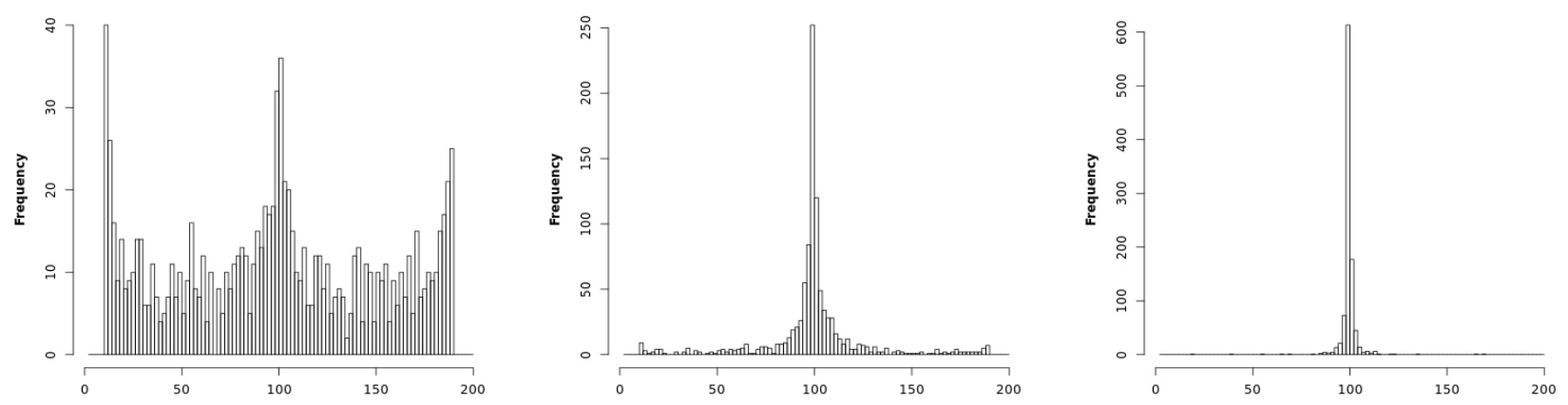

Figure 6: Histogram of the change point estimator $\hat{\theta}$ defined in (3.3). Size $N=200$, data are generated according to model (3.1) with $\theta_{0}=0.5, \mu=0, \delta(t)=a t(1-t)$, for $a=0.1,0.2,0.3$, and the errors are i.i.d. defined by (5.3). The tuning parameter is set to $\varepsilon=0.05$.

\subsection{Results for Covariance operators}

In this section we investigate the finite sample properties of the tests for precise hypotheses regarding the covariance operators as introduced in Section 4.

\subsubsection{Two sample problem}

For the sake of brevity we only display results for fMA(1) processes which are defined by

$$
X_{j}=\eta_{j}+\kappa \eta_{j-1}, \quad Y_{i}=\tilde{\eta}_{i}+\kappa \tilde{\eta}_{i-1} \quad j=1, \ldots m ; i=1, \ldots, n
$$

with $\kappa=0.7$. The error processes are given by

$$
\eta_{j}=\sum_{l=1}^{D} N_{l, j} b_{l} \quad \tilde{\eta}_{i}=a \sum_{l=1}^{D} N_{l, j}^{\prime} b_{l}
$$

for $j=1, \ldots, m ; i=1, \ldots, n$, where the coefficients $N_{i, j}, N_{i, j^{\prime}}^{\prime}$ are independent $N\left(0, \sigma_{i}^{2}\right)(i=$ $\left.1, \ldots, D=21, j=1, \ldots, m, j^{\prime}=1, \ldots, n\right)$ distributed random variables and the (orthonormal) basis functions $b_{1}, \ldots, b_{D}$ are defined in (5.7) $\left(b_{1} \equiv 1\right)$. Similar as in Section 6.3 in Aue et al. (2015), we consider two scenarios for the variance structure of the random coefficients, namely, for any $j=1, \ldots, m, j^{\prime}=1, \ldots, n$,

$$
\begin{aligned}
& \text { (A) } \sigma_{i}^{2}=\operatorname{Var}\left(N_{i, j}\right)=\operatorname{Var}\left(N_{i, j^{\prime}}^{\prime}\right)=1 / i^{2} \quad(i=1, \ldots, D) \\
& \text { (B) } \sigma_{i}^{2}=1.2^{-2 i} \quad(i=1, \ldots, D) \text {. }
\end{aligned}
$$

Note that in this case $X_{i}$ is a multiple of $Y_{j}$ in distribution and the distance between the covariance operators is given by

$$
\int_{0}^{1} \int_{0}^{1} D^{2}(s, t) d s d t=\left(1-a^{2}\right)^{2} \sum_{i=1}^{D} \sigma_{i}^{4}\left(1+\kappa^{2}\right)^{2}
$$


[see Paparoditis and Sapatinas (2016) for a similar approach]. The empirical rejection probabilities of the test (4.2) for different values of $a$ are displayed in Figure 8 , where the case $a=1.5$ corresponds to the boundary of the hypotheses. We observe a similar pattern as for the comparison of the mean functions, where the test 4.2 is slightly more conservative in the variance scenario (A). Additional results with independent data and heavy-tailed errors show a similar picture and can be found in Section A.3 of the online supplement.

\subsubsection{Change point problem}

In this section we investigate the test for a relevant change in the covariance operator, which was developed in Section 4.2. For this purpose we consider an $\mathrm{fMA}(1)$ process $X_{1}^{\prime}, \ldots, X_{N}^{\prime}$ defined by (5.14) (with $\kappa=0.7$ ), where the basis functions and variances $\sigma_{1}^{2}, \ldots, \sigma_{D}^{2}$ are given by (5.7) and (5.15), respectively. The data $X_{1}, \ldots, X_{N}$ is defined by

$$
X_{j}= \begin{cases}X_{j}^{\prime}, & j \leq\left\lfloor N \theta_{0}\right\rfloor \\ a X_{j}^{\prime}, & j>\left\lfloor N \theta_{0}\right\rfloor\end{cases}
$$

for $j=1, \ldots, N$, where the change point is given by $\theta_{0}=0.5$. In Figure 9 we show the rejection probabilities of the test (4.6) for the hypotheses (4.4), where the threshold is given by $\Delta=\left(1-1.5^{2}\right)^{2} \sum_{i=1}^{D} \sigma_{i}^{4}\left(1+0.7^{2}\right)^{2}$. Overall, we observe a similar behaviour as for the test for a relevant change point in the mean functions. Further simulations with independent and heavy-tailed data show similar patterns, see Section A.4 of the online supplement.
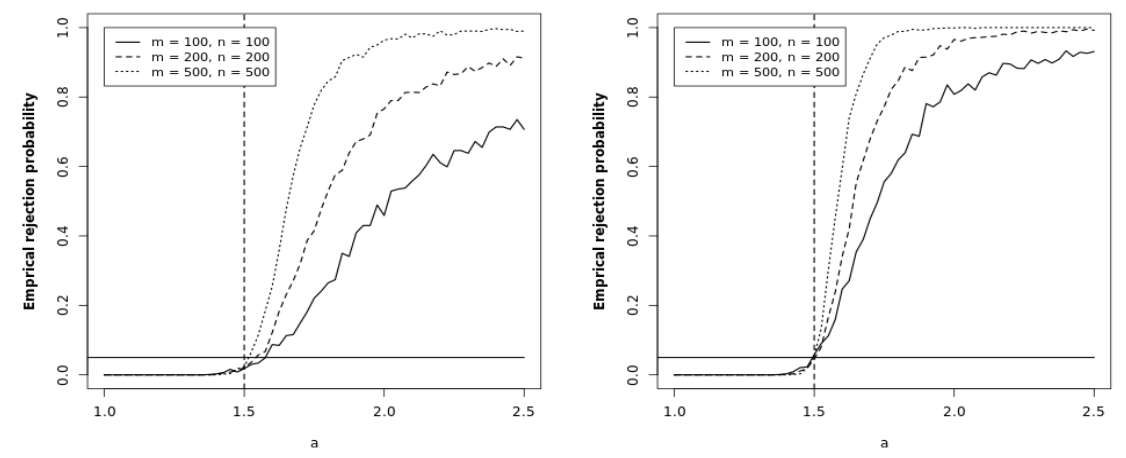

Figure 8: Simulated rejection probabilities of the test (4.2) for the hypotheses (4.1) of a relevant difference between the covariance operators of two $f M A(1)$ processes $\left(\Delta=\left(1-1.5^{2}\right)^{2} \sum_{i=1}^{D} \sigma_{i}^{4}(1+\right.$ $\left.\left.0.7^{2}\right)^{2}\right)$. Left panel: variance scenario (A). Right panel: variance scenario (B). 

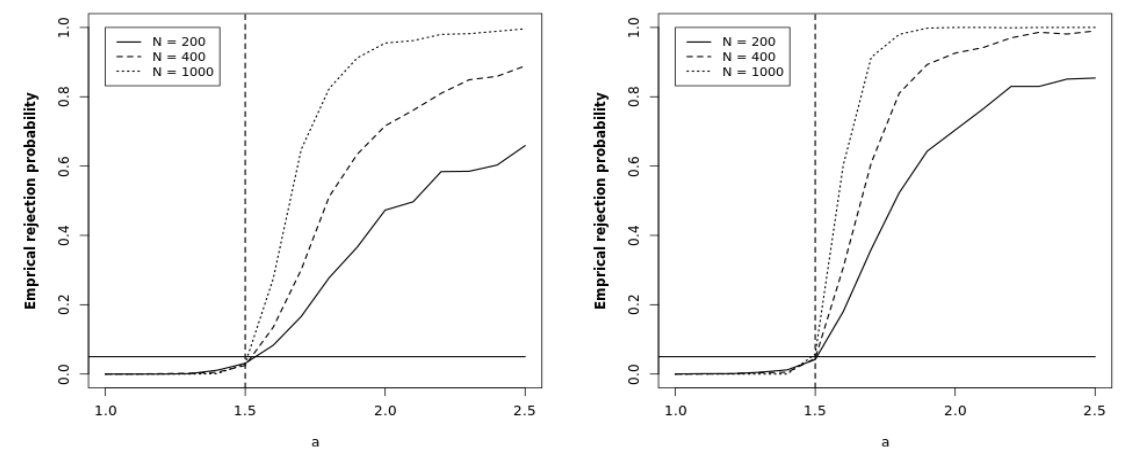

Figure 9: Simulated rejection probabilities of the test (4.6) for the hypotheses (4.4) of a relevant change point in the covariance operator of an $f M A(1)$ process $\left(\Delta=\left(1-1.5^{2}\right)^{2} \sum_{i=1}^{D} \sigma_{i}^{4}\left(1+0.7^{2}\right)^{2}\right)$. Left panel: variance scenario (A). Right panel: variance scenario (B).

\subsection{Data illustrations}

\subsubsection{Two sample test}

In this section we consider an application of the methodology developed in Section 2.2 to Australian temperature data. The data consists of daily minimum temperatures collected at different meteorological stations in Australia. Following Fremdt et al. (2014) we project the daily values of each year on a Fourier basis consisting of 49 basis functions resulting in annual temperature curves for each location under consideration. These authors investigate the temperature data to illustrate methodology designed to choose the dimension of the projection space obtained with fPCA and in Aue and van Delft (2019) the data is considered in the context of stationarity tests for functional time series.

We investigate annual data curves obtained from the meteorological stations in Cape Otway (18652011) and Sydney (1859-2011). Cape Otway is a location in the south of Australia and Sydney is a city on the eastern coast of Australia. There is a distance of approximately $1000 \mathrm{~km}$ between the two locations such that differences in the temperature profiles are expected and the task of the relevant two sample test is now to specify how big the difference might be. The samples consist of $m=147$ and $n=153$ temperature curves, respectively.

In order to calculate the test decision in (2.17) for the hypotheses defined in $(2.16)$, we computed the statistic in 2.18 and the normalizer in 2.19 . We obtained $\hat{\mathbb{D}}_{m, n}=14.115, \hat{\mathbb{V}}_{m, n}=0.315$ and in Table 2, the test decisions are displayed for several choices of the level $\alpha$ and the threshold parameter $\Delta$. In the left panel of Figure 10 we display the two estimated mean functions.

The results in Table 2 provide no evidence for an integrated squared mean difference larger than $\Delta=11.8$ but on the other extreme there is strong evidence that it exceeds $\Delta=9$. Choosing $\Delta$ between 9.1 and 10.7 led to rejecting the null at level $\alpha \geq 5 \%$ and for $\Delta \in[10.8,11.7]$, the test rejected the null only at level $\alpha \geq 10 \%$, which means weaker support of the alternative. 


\begin{tabular}{clll}
\hline$\Delta$ & $99 \%$ & $95 \%$ & $90 \%$ \\
\hline 9.0 & TRUE & TRUE & TRUE \\
9.1 & FALSE & TRUE & TRUE \\
10.7 & FALSE & TRUE & TRUE \\
10.8 & FALSE & FALSE & TRUE \\
11.7 & FALSE & FALSE & TRUE \\
11.8 & FALSE & FALSE & FALSE \\
\hline
\end{tabular}

Table 2: Summary of the two sample test for relevant hypotheses with varying $\Delta$ for the annual temperature curves. The label TRUE refers to a rejection of the null, the label FALSE to a failure to reject the null.

\begin{tabular}{clll}
\hline$\Delta$ & $99 \%$ & $95 \%$ & $90 \%$ \\
\hline 0.72 & FALSE & FALSE & TRUE \\
0.73 & FALSE & FALSE & FALSE \\
\hline
\end{tabular}

Table 3: Summary of the change point test for relevant hypotheses with varying $\Delta$ for the annual river flow curves. The labels TRUE, FALSE refers to a rejection of the null and failure to reject the null, repectively
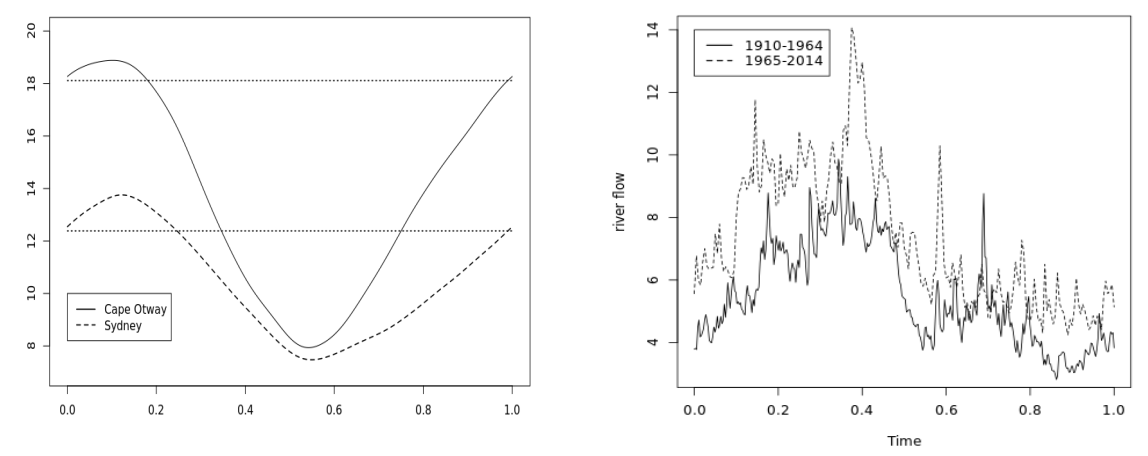

Figure 10: Left panel: Mean functions of the Cape Otway and Sydney series for the two sample case. Right panel: Mean curves of the river flow (in $\mathrm{m}^{3} / \mathrm{sec}$ ) for the periods 1910-1964 and 1965-2014, respectively. 


\subsubsection{Change point test}

In this section we consider daily flows (in $\mathrm{m}^{3} / \mathrm{sec}$ ) of the river Chemnitz at Göritzhain (located in the East of Germany), where data was recorded for the years 1909 - 2014. One hydrological year (different definitions are possible but we consider the same as Sharipov et al. (2014)) starts at the first of November and ends at the 31-th of October which means that we consider the hydrological years 1910-2014. Note that Sharipov et al. (2014) considered the years 1910-2012. We regard data from each year as one flow curve resulting in a sample of size $N=105$.

In the definition of the change point estimator, we use the trimming parameter $\varepsilon=0.1$ and obtain the year 1964 as a possible change point. This is the same year which was identified by Sharipov et al. (2014). In the right panel of Figure 10, we display the mean of the curves before and after the estimated change point. Applying the test defined by (3.8) for two different values of $\Delta$ leads to the test decisions in Table 3 . For $\Delta=0.72$, we reject the null hypothesis of no relevant change at level $\alpha=0.1$ and we do not reject the null at level $\alpha=0.05$. For $\Delta \geq 0.73$, we do not reject the null at the test levels under consideration.

Acknowledgements This work has been supported in part by the Collaborative Research Center "Statistical modeling of nonlinear dynamic processes" (SFB 823, Teilprojekt A1 and C1) of the German Research Foundation (DFG). Parts of this paper were written while H. Dette was visiting the Department of Statistics, University of Toronto, and this author would like to thank the institute for its hospitality. The authors would like to thank Tim Kutta for helpful discussions regarding some of the material on covariance operators. The authors are also grateful to two anonymous referees and the associate editor whose constructive comments lead to a substantial improvement of an earlier version of this manuscript.

\section{References}

Aston, J. A. and Kirch, C. (2012a). Detecting and estimating changes in dependent functional data. Journal of Multivariate Analysis, 109:204-220.

Aston, J. A. and Kirch, C. (2012b). Detecting and estimating changes in dependent functional data. Journal of Multivariate Analysis, 109:204-220.

Aue, A., Dubart Norinho, D., and Hörmann, S. (2015). On the prediction of stationary functional time series. Journal of the American Statistical Association, 110:378-392.

Aue, A., Rice, G., and Sönmez, O. (2018). Structural break analysis for spectrum and trace of covariance operators? arXiv preprint arXiv:1804.03255.

Aue, A. and van Delft, A. (2019). Testing for stationarity of functional time series in the frequency domain. To appear in: Annals of Statistics ArXiv e-print 1701.01741v1.

Benko, M., Haerdle, W., and Kneip, A. (2009). Common functional principal components. Ann. Statist., 37(1):1-34.

Berger, J. O. and Delampady, M. (1987). Testing precise hypotheses. Statistical Science, 2(3):317-335.

Berkes, I., Gabrys, R., Horváth, L., and Kokoszka, P. (2009). Detecting changes in the mean of 
functional observations. Journal of the Royal Statistical Society: Series B (Statistical Methodology), 71(5):927-946.

Berkes, I., Horváth, L., and Rice, G. (2013). Weak invariance principles for sums of dependent random functions. Stochastic Processes and their Applications, 123(2):385 - 403.

Berkson, J. (1938). Some difficulties of interpretation encountered in the application of the chi-square test. Journal of the American Statistical Association, 33(203):526-536.

Bucchia, B. and Wendler, M. (2017). Change-point detection and bootstrap for Hilbert space valued random fields. J. Multivariate Analysis, 155:344-368.

Bücher, A., Dette, H., and Heinrichs, F. (2019). Detecting deviations from second-order stationarity in locally stationary functional time series. To appear in: Annals Institute of the Institute of Statistical Mathematics; arXiv:1808.04092.

Carlstein, E. (1988). Nonparametric change-point estimation. Ann. Statist., 16(1):188-197.

Chow, S.-C. and Liu, P.-J. (1992). Design and Analysis of Bioavailability and Bioequivalence Studies. Marcel Dekker, New York.

Cuevas, A., Febrero, M., and Fraiman, R. (2006). On the use of the bootstrap for estimating functions with functional data. Computational Statistics \& Data Analysis, 51(2):1063 - 1074.

DasGupta, A. (2008). Asymptotic theory of statistics and probability.

Dümbgen, L. (1991). The asymptotic behavior of some nonparametric change-point estimators. The Annals of Statistics, 19(3):1471-1495.

Ferraty, F. and Vieu, P. (2010). Nonparametric Functional Data Analysis. Springer-Verlag, New York. Fremdt, S., Horváth, L., Kokoszka, P., and Steinebach, J. G. (2014). Functional data analysis with increasing number of projections. Journal of Multivariate Analysis, 124:313-332.

Fremdt, S., Steinebach, J., Horvath, L., and Kokoszka, P. (2013). Testing the equality of covariance operators in functional samples. Scandinavian Journal of Statistics, 40(1):138-152.

Fryzlewicz, P. (2014). Wild binary segmentation for multiple change-point detection. Annals of Statistics., 42(6):2243-2281.

Guo, J., Zhou, B., and Zhang, J.-T. (2016). A supremum-norm based test for the equality of several covariance functions. Computational Statistics 83 Data Analysis, 124.

Hall, P. and Van Keilegom, I. (2007). Two-sample tests in functional data analysis starting from discrete data. Statistica Sinica, 17:1511-1531.

Harchaoui, Z. and Lévy-Leduc, C. (2010). Multiple change-point estimation with a total variation penalty. Journal of the American Statistical Association., 105(492):1480-1493.

Hariz, S. B., Wylie, J. J., and Zhang, Q. (2007). Optimal rate of convergence for nonparametric change-point estimators for nonstationary sequences. Ann. Statist., 35(4):1802-1826.

Hörmann, S. and Kokoszka, P. (2010). Weakly dependent functional data. Ann. Statist., 38(3):18451884.

Horváth, L., Kokoska, P., and Reimherr, M. (2009). Two sample inference in functional linear models. The Canadian Journal of Statistics / La Revue Canadienne de Statistique, 37(4):571-591.

Horváth, L. and Kokoszka, P. (2012). Inference for Functional Data with Applications. Springer-Verlag, New York.

Horváth, L., Kokoszka, P., and Reeder, R. (2013). Estimation of the mean of functional time series and 
a two-sample problem. Journal of the Royal Statistical Society: Series B, 75:103-122.

Horváth, L., Kokoszka, P., and Rice, G. (2014). Testing stationarity of functional time series. Journal of Econometrics, 179(1):66 - 82.

Kokoszka, P. (2012). Dependent functional data. ISRN Probability and Statistics, 2012.

Kraus, D. and Panaretos, V. M. (2012). Dispersion operators and resistant second-order functional data analysis. Biometrika, 99(4):813-832.

Munk, A. and Pflüger, R. (1999). (1- $\alpha$ )-equivariant confidence rules for convex alternatives are $\alpha / 2$ level tests - with applications to the multivariate assessment of bioequivalence. Journal of the American Statistical Association, 94(448):1311-1319.

Panaretos, V. M., Kraus, D., and Maddocks, J. H. (2010). Second-order comparison of gaussian random functions and the geometry of dna minicircles. Journal of the American Statistical Association, 105(490):670-682.

Paparoditis, E. and Sapatinas, T. (2016). Bootstrap-based testing of equality of mean functions or equality of covariance operators for functional data. Biometrika, 103(3):727-733.

Pilavakis, D., Paparoditis, E., and Sapatinas, T. (2019). Testing equality of autocovariance operators for functional time series. ArXiv e-print 1901.08535.

Pollard, D. (1984). Convergence of Stochastic Processes. Springer Series in Statistics. Springer New York.

Pomann, G.-M., Staicu, A.-M., and Ghosh, S. (2016). A two-sample distribution-free test for functional data with application to a diffusion tensor imaging study of multiple sclerosis. Journal of the Royal Statistical Society: Series C (Applied Statistics), 65(3):395-414.

Ramsay, J. O. and Silverman, B. W. (2005). Functional Data Analysis. Springer, New York, second edition.

Shao, X. (2010). A self-normalized approach to confidence interval construction in time series. Journal of the Royal Statistical Society: Series B (Statistical Methodology), 72(3):343-366.

Shao, X. (2015). Self-normalization for time series: A review of recent developments. Journal of the American Statistical Association, 110(512):1797-1817.

Shao, X. and Zhang, X. (2010). Testing for change points in time series. Journal of the American Statistical Association, 105(491):1228-1240.

Sharipov, O., Tewes, J., and Wendler, M. (2014). Sequential block bootstrap in a hilbert space with application to change point analysis. Canadian Journal of Statistics, 44.

Sharipov, O. S. and Wendler, M. (2019). Bootstrapping covariance operators of functional time series. arXiv:1904.06721.

Stoehr, C., Aston, J., and Kirch, C. (2019). Detecting changes in the covariance structure of functional time series with application to fMRI data. arXiv:1903.00288.

Van der Vaart, A. W. and Wellner, J. A. (1996). Weak Convergence and Empirical Processes: With Applications in Statistics. Springer, New York.

Volgushev, S. and Shao, X. (2014). A general approach to the joint asymptotic analysis of statistics from sub-samples. Electronic Journal of Statistics, 8(1):390-431.

Vostrikova, L. Y. (1981). Detecting "disorder" in multidimensional random processes. Soviet Mathematics Doklady, 24:55-59. 
Wang, W., Gene Hwang, J., and Dasgupta, A. (1999). Statistical tests for multivariate bioequivalence. Biometrika, 86(2):395-402.

Wellek, S. (2010). Testing Statistical Hypotheses of Equivalence and Noninferiority. CRC Press, Boca Raton, FL, second edition.

Zhang, C., Peng, H., and Zhang, J.-T. (2010). Two samples tests for functional data. Communications in Statistics - Theory and Methods, 39(4):559-578.

Zhang, T. and Lavitas, L. (2018). Unsupervised self-normalized change-point testing for time series. Journal of the American Statistical Association., 113(522):637-648.

Zhang, X. and Shao, X. (2015). Two sample inference for the second-order property of temporally dependent functional data. Bernoulli, 21(2):909-929.

Zhang, X., Shao, X., Hayhoe, K., and Wuebbles, D. J. (2011). Testing the structural stability of temporally dependent functional observations and application to climate projections. Electron. J. Statist., 5:1765-1796. 


\section{Online supplementary material}

This section contains addition finite sample results (Section $\mathrm{A}$ ), proofs of all results in the main part of the paper (Section B) and an extension of the methodology to other testing problems for relevant hypotheses (Section C).

\section{A Additional simulation results}

\section{A.1 Heavy tailed data in the two sample problem}

Here, we display results for the mean functions in 5.8 but as error processes we use

$$
\eta_{j}=\sum_{i=1}^{D} \sqrt{3 /\left(5 i^{2}\right)} t_{i, j} b_{i} \quad(j=1, \ldots, m), \quad \tilde{\eta}_{j}=\sum_{i=1}^{D} \sqrt{3 /\left(5 i^{2}\right)} \tilde{t}_{i, j} b_{i} \quad(j=1, \ldots, n),
$$

where $t_{1,1}, t_{2,1}, \ldots, t_{D, m}, \tilde{t}_{1,1}, \tilde{t}_{2,1}, \ldots, \tilde{t}_{D, n}$ are independent $t_{5}$-distributed random variables and $b_{1}, \ldots, b_{D}$ are B-spline basis functions. Note that the coefficients of the B-splines are defined such that the expectations are zero and the variance of the $i$-th coefficient is equal to $\sigma_{i}^{2}=1 / i^{2}$ $(i=1, \ldots, D)$.

In Figure 11 we show empirical rejection probabilities of the three tests (2.17), (5.9) and (5.10) for different values of $a$. We observe again that all three tests yield very similar rejection probabilities.

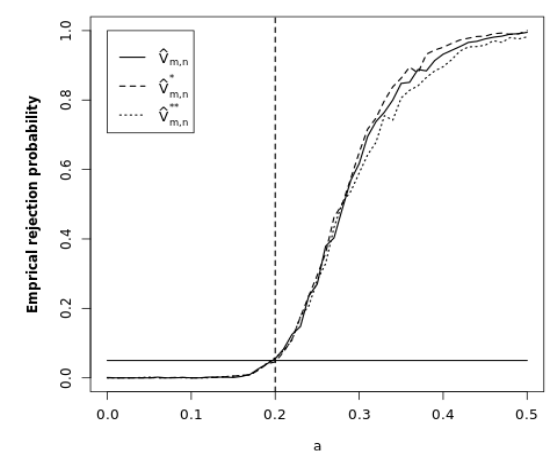

Figure 11: Simulated rejection probabilities of the tests (2.17), (5.9) and (5.10) using different self-normalizing factors. The mean functions are given by (5.8) and two independent samples are considered with sample sizes $m=50, n=100$. The error process is given by A.1.

\section{A.2 Two dependent samples}

In this section we investigate the important case of dependent samples in the two sample problem. We generate a $f M A(1)$ process $\left\{\eta_{i}\right\}_{i \in \mathbb{Z}}$ as described in Section 5.1 and define

$$
X_{i}=\mu_{1}+\eta_{i}, \quad i=1, \ldots, m ; \quad Y_{i}=\mu_{2}+\eta_{m+i}, \quad i=1, \ldots, n
$$


in the first and

$$
X_{i}=\mu_{1}+\eta_{i}, \quad i=1, \ldots, m ; \quad Y_{i}=\mu_{2}+\sqrt{3} \eta_{m+i}, \quad i=1, \ldots, n .
$$

in the second scenario; in both cases $\Delta=0.3^{2} / 30$ and $\mu_{1}, \mu_{2}$ are given in (5.8). The corresponding rejection probabilities of the test (2.17) are depicted in Figure 12. Overall the test performs well in all settings considered.
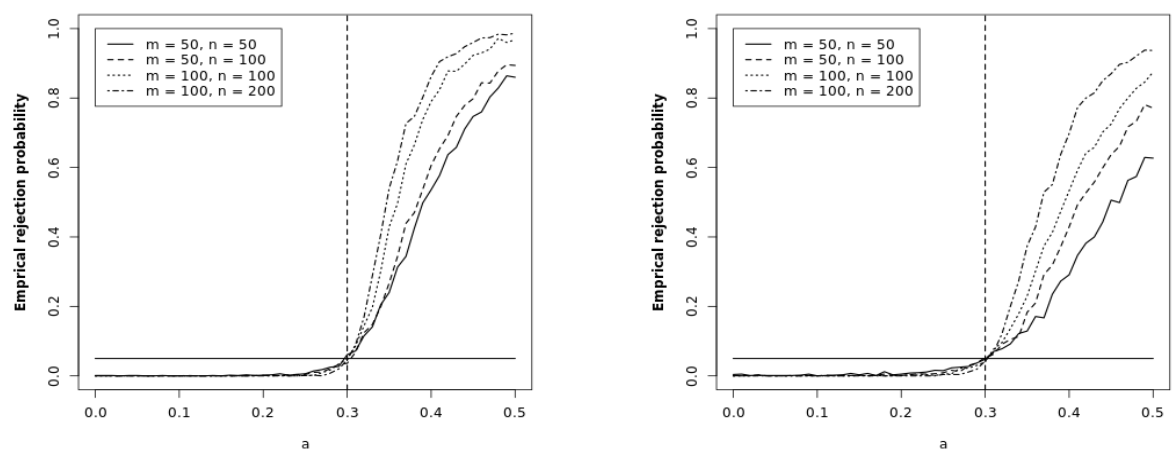

Figure 12: Simulated rejection probabilities of the test (2.17) for the relevant hypotheses (2.16) with $\Delta=0.3^{2} / 30$ in the case of dependent samples. The mean functions are given by (5.8). Left panel: error processes defined by (A.2). Right panel: error processes defined by (A.3).

\section{A.3 The two sample problem for covariance operators}

Recall the setting introduced at the beginning of Section 5.4.1. First, we consider independent data, that is we define

$$
X_{j}=\eta_{j}, \quad Y_{i}=\tilde{\eta}_{i} \quad j=1, \ldots m ; i=1, \ldots, n
$$

In this scenario, we have

$$
\int_{0}^{1} \int_{0}^{1} D^{2}(s, t) d s d t=\left(1-a^{2}\right)^{2} \sum_{i=1}^{D} \sigma_{i}^{4}
$$

and empirical rejection probabilities for different values of $a$ can be seen in Figure 13, while Table 4 shows the simulated level at the boundary of the hypotheses. 

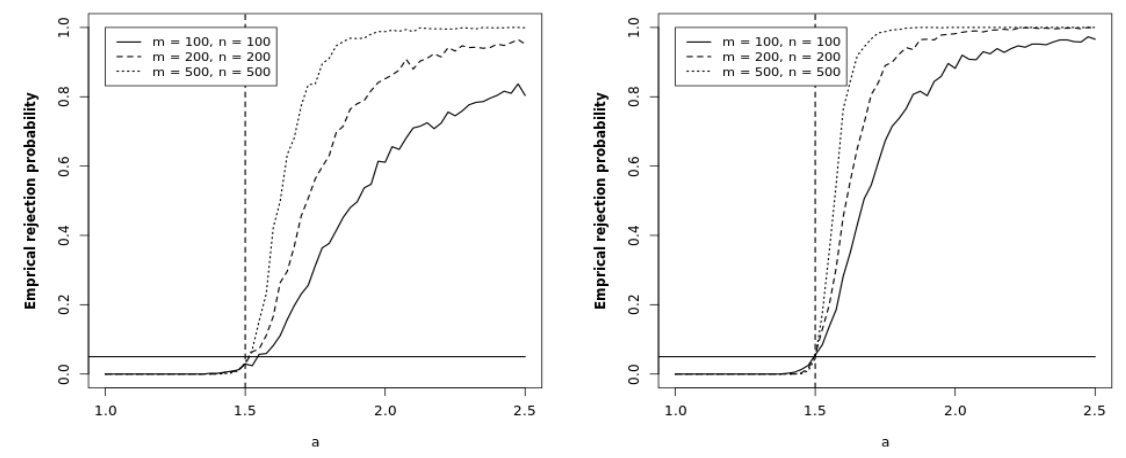

Figure 13: Simulated rejection probabilities of the test (4.2) for the relevant hypotheses (4.1) with $\Delta=\left(1-1.5^{2}\right)^{2} \sum_{i=1}^{D} \sigma_{i}^{4}$. The model is given by A.4). Left panel: variance scenario (A). Right panel: variance scenario $(B)$.

\begin{tabular}{c|ccc||ccc} 
& \multicolumn{3}{|c||}{$(\mathrm{A})$} & \multicolumn{3}{c}{ (B) } \\
\hline & $1 \%$ & $5 \%$ & $10 \%$ & $1 \%$ & $5 \%$ & $10 \%$ \\
\hline \hline$m=n=100$ & 0.4 & 2.9 & 6.7 & 0.8 & 5.5 & 14.5 \\
$m=n=200$ & 0.3 & 2.9 & 7.4 & 1.2 & 5.6 & 12 \\
$m=n=500$ & 0.2 & 3.6 & 8.8 & 1.3 & 5.6 & 11.7
\end{tabular}

Table 4: Approximation of the level at the boundary of the hypotheses in model (A.4).

In Figure 14 we display empirical rejection probabilities for heavy-tailed error processes defined by (5.11), which are similar to those used in Kraus and Panaretos (2012) and in Paparoditis and Sapatinas (2016).

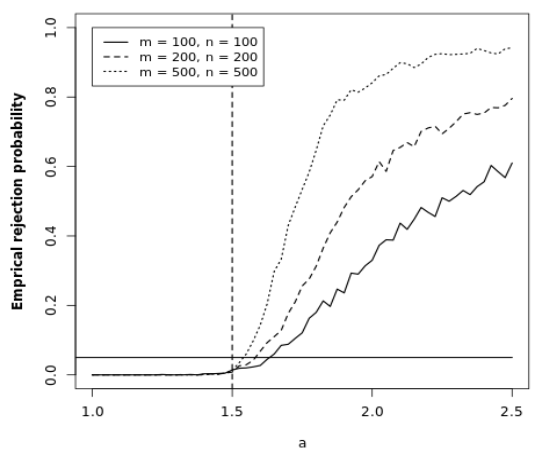

Figure 14: Simulated rejection probabilities of the test (4.2) for the relevant hypotheses (4.1) with $\Delta=1 / 10^{2} \sum_{k=1}^{10}\left(1 / k^{6}+1 / 3^{2 k}\right)\left(1-a^{2}\right)^{2}$. The errors are defined by (5.11). Left panel: variance scenario (A). Right panel: variance scenario (B). 


\section{A.4 Change point problems for covariance operators}

The setting considered here is the same as in Section 5.4.2. In Figure 15, we consider independent data as in (A.4) and display the empirical rejection probabilities for different values of $a$ and fixed threshold $\Delta=\left(1-1.5^{2}\right)^{2} \sum_{i=1}^{D} \sigma_{i}^{4}$. In Figure 16 , we display empirical rejection probabilities for data with heavy-tailed errors as in (5.11). In this scenario the threshold parameter $\Delta$ is fixed to $\Delta=1 / 10^{2} \sum_{k=1}^{10}\left(1 / k^{6}+1 / 3^{2 k}\right)\left(1-a^{2}\right)^{2}$.
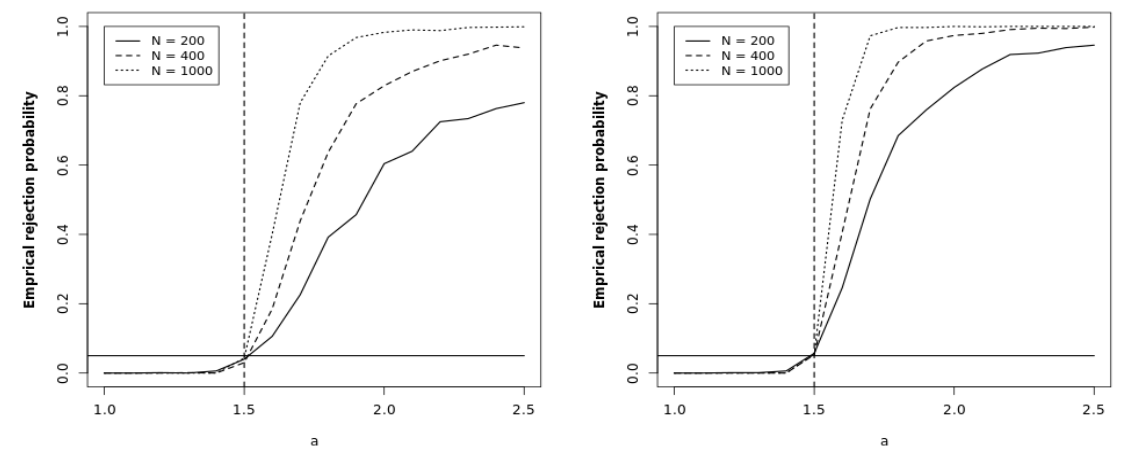

Figure 15: Simulated rejection probabilities of the test (4.6) for the relevant hypotheses (4.4) with $\Delta=\left(1-1.5^{2}\right)^{2} \sum_{i=1}^{D} \sigma_{i}^{4}$. The model is given by A.4). Left panel: variance scenario (A). Right panel: variance scenario $(B)$.

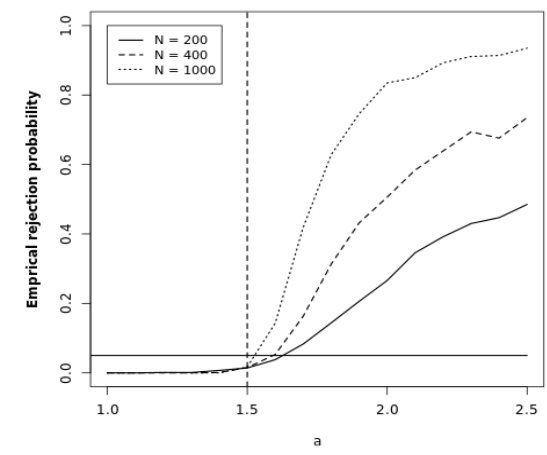

Figure 16: Simulated rejection probabilities of the test (4.6) for the relevant hypotheses (4.4) with $\Delta=\left(1-1.5^{2}\right)^{2} \sum_{i=1}^{D} \sigma_{i}^{4}$. The errors are given by (5.11).

\section{A.5 Comparison of self-normalization with long-run variance estima- tion for heavy-tailed data}

This section contains additional results comparing the performance of self-normalization and estimated long-run variance. First, we consider rejection probabilities under the null but for 
fAR(1) processes with heavy-tailed errors. More precisely we replace the errors $\eta_{j}$ in $(5.6)$ by

$$
\eta_{j}^{\prime}=\sum_{i=1}^{D} \sqrt{3 /\left(5 i^{2}\right)} t_{i, j} b_{i} \quad(j=1, \ldots, n)
$$

where $t_{1,1}, t_{2,1}, \ldots, t_{D, n}$ are independent $t_{5}$-distributed random variables. The results are shown in Figure 17 and we observe that the self-normalized test yields a much better approximation of the nominal level.
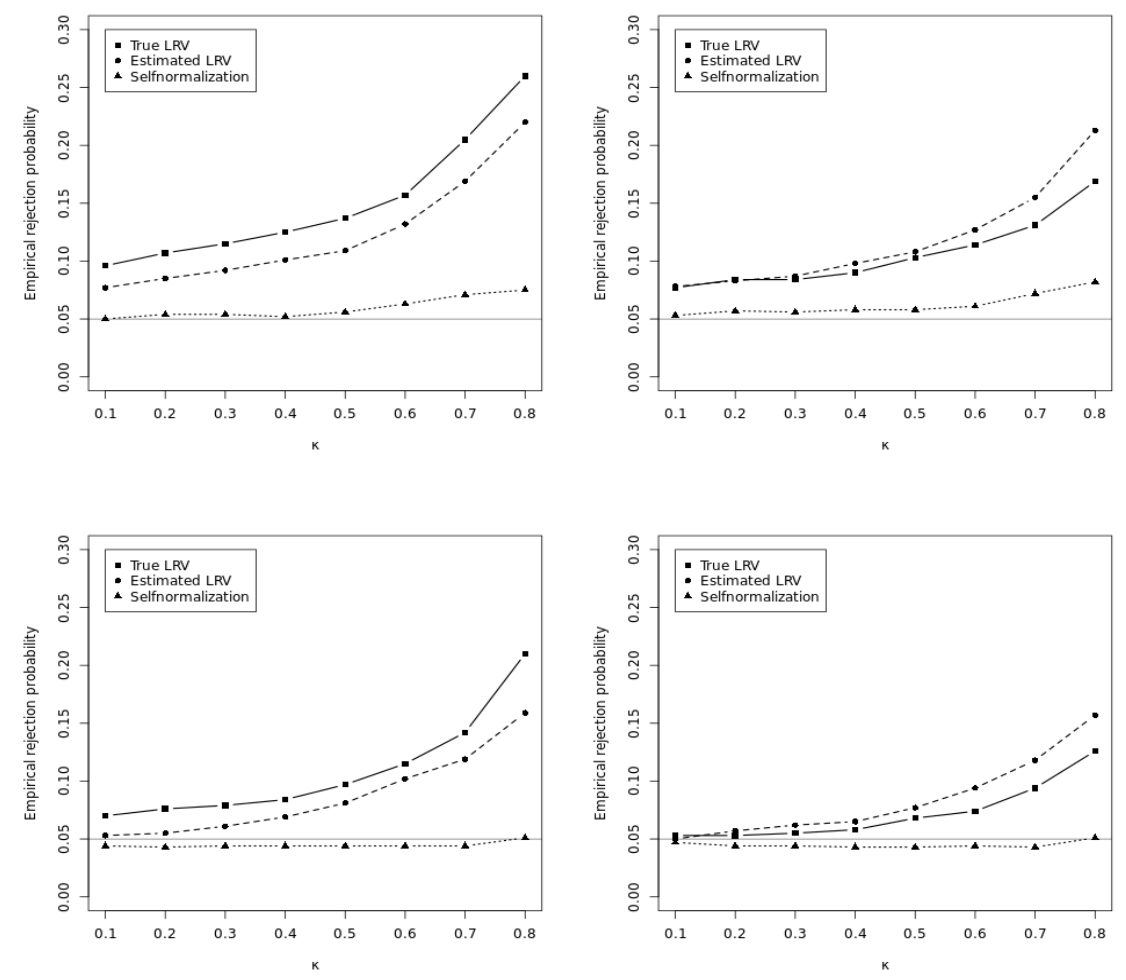

Figure 17: Approximation of the test level for different values of $\kappa$. Errors are heavy-tailed fAR(1) processes defined by (A.5). Top left: $\delta=0.5, n=100$; Top right: $\delta=1.5, n=100$; Bottom left: $\delta=0.5, n=200$; Bottom right: $\delta=1.5, n=200$ 


\section{B Proofs}

We begin with some technical preliminaries, the notation introduced in this section will be used throughout all the proofs. Define the set of functions

$$
\mathcal{G}:=\left\{f: T \times[0,1] \rightarrow \mathbb{R}: \sup _{\lambda \in[0,1]} \int_{T} f^{2}(t, \lambda) d t<\infty\right\} .
$$

Equip this set with the norm

$$
\|f-g\|_{\mathcal{G}}:=\sup _{\lambda \in[0,1]}\left\{\int_{T}[f(t, \lambda)-g(t, \lambda)]^{2} d t\right\}^{1 / 2}
$$

to obtain a normed vector space. We will frequently work with random elements with values in this space. Here, random elements need not be measurable and we will make use of the general theory of outer probabilities (see Chapter 1 in Van der Vaart and Wellner (1996)) where appropriate without explicitly mentioning this.

\section{B.1 Proofs of the results in Section 2}

First, we state some preliminary results that will be useful throughout the proofs. Define

$$
\tilde{S}_{n}(t, \lambda)=\frac{1}{n} \sum_{j=1}^{\lfloor n \lambda\rfloor}\left(X_{j}(t)-\mu(t)\right), \quad \lambda \in[0,1] .
$$

Then it follows from Theorem 1.1 in Berkes et al. (2013), that there exists a sequence of measurable random elements in $\mathcal{G}$, say $\left\{\Gamma_{n}(t, \lambda)\right\}_{\lambda, t \in[0,1]}$, such that

$$
\begin{aligned}
& \sup _{\lambda \in[0,1]} \int_{T}\left(\sqrt{n} \tilde{S}_{n}(t, \lambda)-\Gamma_{n}(t, \lambda)\right)^{2} d t=o_{\mathbb{P}}(1) \\
& \left\{\Gamma_{n}(t, \lambda)\right\}_{\lambda, t \in[0,1]} \stackrel{\mathcal{D}}{=}\{\Gamma(t, \lambda)\}_{\lambda, t \in[0,1]}
\end{aligned}
$$

where $\Gamma$ is defined by

$$
\Gamma(t, \lambda)=\sum_{i=1}^{\infty} \sqrt{\lambda_{i}} \phi_{i}(t) W_{i}(\lambda) .
$$

$\left\{W_{i}\right\}_{i \in \mathbb{N}}$ is a sequence of independent Brownian motions and $\lambda_{i}, \phi_{i}$ are the eigenvalues and (orthonormal) eigenfunctions of the integral operator corresponding to the covariance kernel

$$
C(s, t)=\sum_{i=1}^{\infty} \lambda_{i} \phi_{i}(s) \phi_{i}(t)
$$

defined in 2.5 , that is

$$
\lambda_{i} \phi_{i}(s)=\int_{T} C(t, s) \phi_{i}(t) d t \quad(i \in \mathbb{N})
$$


Note that Berkes et al. (2013) also prove that $\sum_{k} \lambda_{k}<\infty$ (see their Lemma 2.2) and that

$$
\sup _{0 \leq \lambda \leq 1} \int_{T} \Gamma^{2}(t, \lambda) d t<\infty \quad \text { a.s. }
$$

The latter implies that for any square integrable function $\zeta:[0,1] \rightarrow \mathbb{R}$ the process

$$
\left\{\int_{T} \zeta(t) \Gamma(t, \lambda) d t\right\}_{\lambda \in[0,1]}
$$

can be viewed as an element of $\ell^{\infty}([0,1])$ and that the same is true for the process $\left\{\int_{T} \Gamma^{2}(t, \lambda) d t\right\}_{\lambda \in[0,1]}$. Moreover, summability of the sequence $\left(\lambda_{k}\right)_{k \in \mathbb{N}}$ together with properties of the modulus of continuity of Brownian motions implies that for any positive sequence $\left(\kappa_{k}\right)_{k \in \mathbb{N}}$ such that $\kappa_{n} \rightarrow 0$, it follows

$$
\begin{aligned}
& \sup _{\substack{\nu, \lambda \in[0,1]: \\
|\nu-\lambda| \leq \kappa_{n}}} \int_{T}\{\Gamma(t, \lambda)-\Gamma(t, \nu)\}^{2} d t \\
= & \sup _{\substack{\nu, \lambda \in[0,1]: \\
|\nu-\lambda| \leq \kappa_{n}}} \int_{T} \sum_{i=1}^{\infty} \sum_{j=1}^{\infty} \sqrt{\lambda_{i} \lambda_{j}}\left\{W_{i}(\lambda)-W_{i}(\nu)\right\}\left\{W_{j}(\lambda)-W_{j}(\nu)\right\} \phi_{i}(t) \phi_{j}(y) d t \\
= & \sup _{\substack{\nu, \lambda \in[0,1]: \\
|\nu-\lambda| \leq \kappa_{n}}} \sum_{i=1}^{\infty} \sum_{j=1}^{\infty} \sqrt{\lambda_{i} \lambda_{j}}\left\{W_{i}(\lambda)-W_{i}(\nu)\right\}\left\{W_{j}(\lambda)-W_{j}(\nu)\right\} \int_{T} \phi_{i}(t) \phi_{j}(y) d t \\
= & \sup _{\substack{\nu, \lambda \in[0,1]: \\
|\nu-\lambda| \leq \kappa_{n}}} \sum_{i=1}^{\infty} \lambda_{i}\left\{W_{i}(\lambda)-W_{i}(\nu)\right\}^{2} \\
\leq & \sum_{i=1}^{\infty} \lambda_{i} \sup _{\substack{\nu, \lambda \in[0,1]: \\
|\nu-\lambda| \leq \kappa_{n}}}\left\{W_{i}(\lambda)-W_{i}(\nu)\right\}^{2}=o_{\mathbb{P}}(1)
\end{aligned}
$$

where the last line follows since by Fubini's Theorem

$$
\mathbb{E}\left[\sum_{i=1}^{\infty} \lambda_{i} \sup _{\substack{\nu, \lambda \in[0,1]: \\|\nu-\lambda| \leq \kappa_{n}}}\left\{W_{i}(\lambda)-W_{i}(\nu)\right\}^{2}\right]=\mathbb{E}\left[\sup _{\substack{\nu, \lambda \in[0,1]: \\|\nu-\lambda| \leq \kappa_{n}}}\left\{W_{1}(\lambda)-W_{1}(\nu)\right\}^{2}\right] \sum_{i=1}^{\infty} \lambda_{i}=o(1) .
$$

This implies

$$
\sup _{\substack{\nu, \lambda \in[0,1]: \\|\nu-\lambda| \leq \kappa_{n}}} \int_{T}\left\{\Gamma^{2}(t, \lambda)-\Gamma^{2}(t, \nu)\right\}^{2} d t=o_{\mathbb{P}}(1) \quad \kappa_{n} \rightarrow 0
$$

\section{B.1.1 Proof of Theorem 2.1}

The main ingredients of the proof are the convergence result stated in 2.11 when $\int_{T} \mu^{2}(t) d t>0$ and the bounds $\hat{\mathbb{T}}_{n}=o_{\mathbb{P}}(1), \hat{\mathbb{V}}_{n}=o_{\mathbb{P}}(1)$ when $\int_{T} \mu^{2}(t) d t=0$. We begin by considering 
the case $\int_{T} \mu^{2}(t) d t=0$. In that case we have $\tilde{S}_{n}(t, \lambda) \equiv S_{n}(t, \lambda)$ for and hence by (B.2) $\sup _{\lambda \in[0,1]} \int_{T} S_{n}^{2}(t, \lambda) d t=o_{\mathbb{P}}(1)$ which implies $\hat{\mathbb{T}}_{n}=o_{\mathbb{P}}(1), \hat{\mathbb{V}}_{n}=o_{\mathbb{P}}(1)$.

For the case $\int_{T} \mu^{2}(t) d t>0$ note that a straightforward calculation shows

$$
\begin{aligned}
\sqrt{n} \int_{T}\left(S_{n}^{2}(t, \lambda)-\lambda^{2} \mu^{2}(t)\right) d t= & \sqrt{n} \int_{T}\left(S_{n}(t, \lambda)-\lambda \mu(t)\right)^{2} d t+2 \sqrt{n} \int_{T} \lambda \mu(t)\left(S_{n}(t, \lambda)-\lambda \mu(t)\right) d t \\
= & \sqrt{n} \int_{T} \tilde{S}_{n}^{2}(t, \lambda) d t+2 \sqrt{n} \int_{T} \lambda \mu(t) \tilde{S}_{n}(t, \lambda) d t+o_{\mathbb{P}}(1) \\
= & \frac{1}{\sqrt{n}} \int_{T}\left(\sqrt{n} \tilde{S}_{n}(t, \lambda)-\Gamma_{n}(t, \lambda)\right)^{2} d t+\frac{1}{\sqrt{n}} \int_{T} \Gamma_{n}^{2}(t, \lambda) d t \\
& -\frac{2}{\sqrt{n}} \int_{T}\left(\sqrt{n} \tilde{S}_{n}(t, \lambda)-\Gamma_{n}(t, \lambda)\right) \Gamma_{n}(t, \lambda) d t \\
& +2 \int_{T} \lambda \mu(t)\left(\sqrt{n} \tilde{S}_{n}(t, \lambda)-\Gamma_{n}(t, \lambda)\right) d t+2 \int_{T} \lambda \mu(t) \Gamma_{n}(t, \lambda) d t+o_{\mathbb{P}}(1) \\
= & 2 \int_{T} \lambda \mu(t) \Gamma_{n}(t, \lambda) d t+o_{\mathbb{P}}(1)
\end{aligned}
$$

uniformly with respect to $\lambda \in[0,1]$, where we repeatedly used $(\overline{B .2})$ and the Cauchy-Schwarz inequality. Therefore we obtain from (B.3) and Slutsky's Lemma that

$$
\left\{\sqrt{n}\left(\int_{T} S_{n}^{2}(t, \lambda) d t-\lambda^{2} \int_{T} \mu^{2}(t) d t\right)\right\}_{\lambda \in[0,1]} \rightsquigarrow\left\{2 \lambda \int_{T} \mu(t) \Gamma(t, \lambda) d t\right\}_{\lambda \in[0,1]}
$$

in $\ell^{\infty}([0,1])$ (recall that by the discussion at the beginning of this section the process on the right hand side is an element of $\left.\ell^{\infty}([0,1])\right)$, and observing (B.4), (B.5) and (B.6) it follows by a straightforward calculation that

$$
\operatorname{Cov}\left(\int_{T} \mu(t) \Gamma(t, \lambda) d t, \int_{T} \mu(t) \Gamma\left(t, \lambda^{\prime}\right) d t\right)=\left(\lambda \wedge \lambda^{\prime}\right) \int_{T} \int_{T} \mu(t) \mu(s) C(s, t) d s d t .
$$

Therefore

$$
\left\{2 \lambda \int_{T} \mu(t) \Gamma(t, \lambda) d t\right\}_{\lambda \in[0,1]} \stackrel{\mathcal{D}}{=}\{\lambda \tau \mathbb{B}(\lambda)\}_{\lambda \in[0,1]},
$$

where $\mathbb{B}$ denotes a standard Brownian motion on the interval $[0,1]$ and $\tau^{2}$ is defined in (2.4). Consequently the statement (2.11) in Section 2 follows from (B.9) and an application of the continuous mapping theorem observing that the mapping

$$
\mathbb{Z} \mapsto \frac{\mathbb{Z}(1)}{\left(\int_{0}^{1}\left(\mathbb{Z}(\lambda)-\lambda^{2} \mathbb{Z}(1)\right)^{2} \nu(d \lambda)\right)^{1 / 2}}
$$

from the measurable functions in $\ell^{\infty}([0,1])$ onto $\mathbb{R}$ is continuous at points $f \in \ell^{\infty}([0,1])$ with $\int_{0}^{1}\left(f(\lambda)-\lambda^{2} f(1)\right)^{2} \nu(d \lambda) \neq 0$. This yields

$$
\frac{\hat{\mathbb{T}}_{n}-d}{\hat{\mathbb{V}}_{n}} \stackrel{\mathcal{D}}{\rightarrow} \frac{\mathbb{B}(1)}{\left(\int_{0}^{1} \lambda^{2}(\mathbb{B}(\lambda)-\lambda \mathbb{B}(1))^{2} \nu(d \lambda)\right)^{1 / 2}}=\mathbb{W} .
$$


In the discussion following Theorem 2.1 we already argued that

$$
\lim _{n \rightarrow \infty} \mathbb{P}\left(\hat{\mathbb{T}}_{n}>\Delta+q_{1-\alpha}(\mathbb{W}) \hat{\mathbb{V}}_{n}\right)=0,
$$

whenever $\int_{T} \mu^{2}(t) d t=0$. When $0<\int_{T} \mu^{2}(t) d t<\Delta$, we have

$$
\lim _{n \rightarrow \infty} \mathbb{P}\left(\hat{\mathbb{T}}_{n}>\Delta+q_{1-\alpha}(\mathbb{W}) \hat{\mathbb{V}}_{n}\right)=\lim _{n \rightarrow \infty} \mathbb{P}\left(\frac{\hat{\mathbb{T}}_{n}-d}{\hat{\mathbb{V}}_{n}}>\frac{\sqrt{n}(\Delta-d)}{\sqrt{n} \hat{\mathbb{V}}_{n}}+q_{1-\alpha}(\mathbb{W})\right)=0
$$

since $\sqrt{n} \hat{\mathbb{V}}_{n}=O_{\mathbb{P}}(1), \frac{\hat{\mathbb{T}}_{n}-d}{\hat{\mathbb{V}}_{n}}=O_{\mathbb{P}}(1), \hat{\mathbb{V}}_{n} \geq 0$ a.s. and $\sqrt{n}(\Delta-d) \rightarrow+\infty$. In the case $d=$ $\int_{T} \mu^{2}(t) d t=\Delta$ we conclude

$$
\lim _{n \rightarrow \infty} \mathbb{P}\left(\hat{\mathbb{T}}_{n}>\Delta+q_{1-\alpha}(\mathbb{W}) \hat{\mathbb{V}}_{n}\right)=\lim _{n \rightarrow \infty} \mathbb{P}\left(\frac{\hat{\mathbb{T}}_{n}-d}{\hat{\mathbb{V}}_{n}}>q_{1-\alpha}(\mathbb{W})\right)=\alpha
$$

and, if $d>\Delta$, we have

$$
\lim _{n \rightarrow \infty} \mathbb{P}\left(\hat{\mathbb{T}}_{n}>\Delta+q_{1-\alpha}(\mathbb{W}) \hat{\mathbb{V}}_{n}\right)=\lim _{n \rightarrow \infty} \mathbb{P}\left(\hat{\mathbb{T}}_{n}-d>\Delta-d+q_{1-\alpha}(\mathbb{W}) \hat{\mathbb{V}}_{n}\right)=1
$$

since $\hat{\mathbb{V}}_{n}=o_{\mathbb{P}}(1), \hat{\mathbb{T}}_{n}-d==o_{\mathbb{P}}(1)$ and $(\Delta-d)<0$.

\section{B.1.2 Proof of Theorem 2.2}

The processes $\left\{X_{m}\right\}_{m \in \mathbb{Z}}$ and $\left\{Y_{n}\right\}_{n \in \mathbb{Z}}$ satisfy assumptions (B2) and thus admit the representation $X_{j}=\mu_{1}+\eta_{j}^{X}, Y_{j}=\mu_{2}+\eta_{j}^{Y}$ where $\left(\eta_{j}^{X}\right)_{j \in \mathbb{Z}}$ and $\left(\eta_{j}^{Y}\right)_{j \in \mathbb{Z}}$ denote centered error processes that both satisfy (A2)-(A4). Define

$$
\tilde{D}_{m, n}(t, \lambda):=S_{m}^{X}(t, \lambda)-S_{n}^{Y}(t, \lambda),
$$

where the processes $S_{m}^{X}$ and $S_{n}^{Y}$ are given by

$$
\begin{aligned}
& S_{m}^{X}(t, \lambda)=\frac{1}{m} \sum_{j=1}^{\lfloor m \lambda\rfloor}\left(X_{j}(t)-\mu_{1}(t)\right)=\frac{1}{m} \sum_{j=1}^{\lfloor m \lambda\rfloor} \eta_{j}^{X}(t), \\
& S_{n}^{Y}(t, \lambda)=\frac{1}{n} \sum_{j=1}^{\lfloor n \lambda\rfloor}\left(Y_{j}(t)-\mu_{2}(t)\right)=\frac{1}{n} \sum_{j=1}^{\lfloor n \lambda\rfloor} \eta_{j}^{Y}(t),
\end{aligned}
$$

respectively. A similar calculation as given in Section B.1.1 shows that

$$
\begin{aligned}
\mathbb{Z}_{m, n}(\lambda) & :=\sqrt{n+m} \int_{T}\left(D_{m, n}^{2}(t, \lambda)-\lambda^{2} D^{2}(t)\right) d t \\
& =2 \sqrt{n+m} \int_{T} \lambda D(t)\left(D_{m, n}(t, \lambda)-\lambda D(t)\right) d t+o_{\mathbb{P}}(1) \\
& =2 \sqrt{n+m} \int_{T} \lambda D(t) \tilde{D}_{m, n}(t, \lambda) d t+o_{\mathbb{P}}(1)=\mathbb{Z}_{m}^{X}(\lambda)-\mathbb{Z}_{n}^{Y}(\lambda)+o_{\mathbb{P}}(1),
\end{aligned}
$$


where we use the fact that $\lambda \mu_{1}=\frac{1}{m} \sum_{i=1}^{\lfloor m \lambda\rfloor} \mu_{1}+o(1)$ uniformly in $\lambda$ in the third equality and the processes $\left\{\mathbb{Z}_{m}^{X}(\lambda)\right\}_{\lambda \in[0,1]}$ and $\left\{\mathbb{Z}_{n}^{Y}(\lambda)\right\}_{\lambda \in[0,1]}$ are given by

$$
\begin{aligned}
& \mathbb{Z}_{m}^{X}(\lambda):=2 \sqrt{n+m} \int_{T} \lambda D(t) S_{m}^{X}(t, \lambda) d t, \\
& \mathbb{Z}_{n}^{Y}(\lambda):=2 \sqrt{n+m} \int_{T} \lambda D(t) S_{n}^{Y}(t, \lambda) d t,
\end{aligned}
$$

respectively. As the times series $\left\{X_{n}\right\}_{n \in \mathbb{Z}}$ and $\left\{Y_{n}\right\}_{n \in \mathbb{Z}}$ satisfy assumptions (A1) - (A4) it follows from the proof of Theorem 2.1 that the processes $\left\{\mathbb{Z}_{m}^{X}(\lambda)\right\}_{\lambda \in[0,1]}$ and $\left\{\mathbb{Z}_{n}^{Y}(\lambda)\right\}_{\lambda \in[0,1]}$ converge weakly in $\ell^{\infty}([0,1])$ with both limits corresponding to scaled Brownian motions. Furthermore, both processes are independent and therefore

$$
\left\{\mathbb{Z}_{m, n}(\lambda)\right\}_{\lambda \in[0,1]} \rightsquigarrow\left\{\lambda \tau_{D} \mathbb{B}(\lambda)\right\}_{\lambda \in[0,1]}
$$

in $\ell^{\infty}([0,1])$, where $\{\mathbb{B}(\lambda)\}_{\lambda \in[0,1]}$ is a Brownian motion and $\tau_{D}$ is a real number depending on the auto-covariance structures of $\left\{X_{m}\right\}_{m \in \mathbb{Z}}$ and $\left\{Y_{n}\right\}_{n \in \mathbb{Z}}$.

The assertion now follows exactly in the same way as in the proof of Theorem 2.1 and the details are omitted for the sake of brevity.

\section{B.2 Proofs of the results in Section 3}

\section{B.2.1 A technical result}

In this section we prove a useful intermediate result. Now consider the situation which is described in model (3.1) (see also Remark 2.4(a)). Set

$$
\begin{array}{ll}
\eta_{i}^{(1)}=f_{1}\left(\varepsilon_{i}, \varepsilon_{i-1, \ldots}\right), & i=1, \ldots, N \\
\eta_{i}^{(2)}=f_{2}\left(\varepsilon_{i}, \varepsilon_{i-1, \ldots}\right), & i=1, \ldots, N
\end{array}
$$

where $f_{1}, f_{2},\left(\varepsilon_{j}\right)_{j \in \mathbb{Z}}$ satisfy the conditions in $(\mathrm{A} 2), \mathbb{E}\left[\eta_{1}^{(1)}\right]=\mathbb{E}\left[\eta_{1}^{(2)}\right]=0$, and $\eta_{i}^{(1)}, \eta_{i}^{(2)}$ satisfy assumptions (A3), (A4).

Lemma B.1 In the setting above consider a fixed (but arbitrary) function $\zeta$ in $L^{2}(T)$. For $\lambda \in[0,1]$ define the processes

$$
\tilde{Z}_{N}^{(k)}(\lambda):=\frac{1}{\sqrt{N}} \sum_{i=1}^{\lfloor N \lambda\rfloor} \int_{T} \eta_{i}^{(k)}(t) \zeta(t) d t, \quad k=1,2,
$$

then

$$
\left(\tilde{Z}_{N}^{(1)}, \tilde{Z}_{N}^{(2)}\right)^{\top} \rightsquigarrow \Sigma^{1 / 2}\left(\mathbb{B}_{1}, \mathbb{B}_{2}\right)^{\top} \quad \text { in } \ell^{\infty}([0,1])^{2},
$$

where $\mathbb{B}_{1}, \mathbb{B}_{2}$ are two independent standard Brownian motions on the interval $[0,1]$ and $\Sigma$ is a symmetric $2 \times 2$ matrix with finite entries given by

$$
\Sigma_{i j}=\sum_{h \in \mathbb{Z}} \int_{T} \int_{T} \operatorname{Cov}\left(\eta_{0}^{(i)}(s), \eta_{h}^{(j)}(t)\right) \zeta(s) \zeta(t) d s d t .
$$


Proof It suffices to prove tightness of the processes $\tilde{Z}_{N}^{(1)}, \tilde{Z}_{N}^{(2)}$ individually and joint finitedimensional convergence. Tightness of $\tilde{Z}_{N}^{(k)}$ follows from weak convergence of $\tilde{Z}_{N}^{(k)}$, which can be established by an application of Theorem 2.1 in Berkes et al. (2013) and similar arguments as given in Section B.1.1. Thus it remains to prove that for arbitrary $s_{1}, \ldots, s_{L} \in[0,1]$ we have

$$
\left(\tilde{Z}_{N}^{(1)}\left(s_{1}\right), \tilde{Z}_{N}^{(2)}\left(s_{1}\right), \ldots, \tilde{Z}_{N}^{(1)}\left(s_{L}\right), \tilde{Z}_{N}^{(2)}\left(s_{L}\right)\right) \rightsquigarrow \mathcal{N}\left(0, \Sigma\left(s_{1}, \ldots, s_{L}\right)\right)
$$

where $\Sigma\left(s_{1}, \ldots, s_{L}\right)$ denotes the covariance matrix of the vector $\left(\mathbb{G}_{1}\left(s_{1}\right), \mathbb{G}_{2}\left(s_{1}\right), \ldots, \mathbb{G}_{1}\left(s_{L}\right), \mathbb{G}_{2}\left(s_{L}\right)\right)$ and $\left(\mathbb{G}_{1}, \mathbb{G}_{2}\right)^{\top}:=\Sigma\left(\mathbb{B}_{1}, \mathbb{B}_{2}\right)^{\top}$. Following Berkes et al. (2013) we define the random variables

$$
\eta_{j, m}^{(k)}:=f_{k}\left(\varepsilon_{j}, \varepsilon_{j-1}, \ldots \varepsilon_{j-m+1}, \varepsilon_{j, m}^{*}\right), \quad k=1,2, m \in \mathbb{N}
$$

where $\varepsilon_{j, m}^{*}=\left(\varepsilon_{j, m, j-m}^{*}, \varepsilon_{j, m, j-m-1}^{*}, \ldots\right)$ is given in Assumption (A4). Let $\Sigma_{m}$ denote matrices with entries (below we shall prove that all entries are finite for any $m \geq 1$ )

$$
\left(\Sigma_{m}\right)_{i j}:=\sum_{|h| \leq m} \int_{T} \int_{T} \operatorname{Cov}\left(\eta_{0, m}^{(i)}(s), \eta_{h, m}^{(j)}(t)\right) \zeta(s) \zeta(t) d s d t
$$

define $\left(\mathbb{G}_{1, m}, \mathbb{G}_{2, m}\right)^{\top}:=\Sigma_{m}^{1 / 2}\left(\mathbb{B}_{1}, \mathbb{B}_{2}\right)^{\top}$ and

$$
\tilde{Z}_{N, m}^{(k)}(\lambda):=\frac{1}{\sqrt{N}} \sum_{i=1}^{\lfloor N \lambda\rfloor} \int_{T} \eta_{i, m}^{(k)}(t) \zeta(t) d t, \quad k=1,2 .
$$

By an application of Example 11 in Chapter IV of Pollard (1984) it suffices to prove that

(i) $\Sigma_{m} \rightarrow \Sigma$ as $m \rightarrow \infty$.

(ii) For any $\delta>0, k \in\{1,2\}, \ell \in\{1, \ldots, L\}$ we have

$$
\lim _{m \rightarrow \infty} \limsup _{N \rightarrow \infty} \mathbb{P}\left(\left|\tilde{Z}_{N, m}^{(k)}\left(s_{\ell}\right)-\tilde{Z}_{N}^{(k)}\left(s_{\ell}\right)\right|>\delta\right)=0 .
$$

(iii) For any fixed $m$ we have

$$
\left(\tilde{Z}_{N, m}^{(1)}\left(s_{1}\right), \tilde{Z}_{N, m}^{(2)}\left(s_{1}\right), \ldots, \tilde{Z}_{N, m}^{(1)}\left(s_{L}\right), \tilde{Z}_{N, m}^{(2)}\left(s_{L}\right)\right) \rightsquigarrow \mathcal{N}\left(0, \Sigma_{m}\left(s_{1}, \ldots, s_{L}\right)\right)
$$

In order to show the claim in (i), we prove $\left(\Sigma_{m}\right)_{i j} \rightarrow \Sigma_{i j}$ as $m \rightarrow \infty$ for $i, j=1,2$. For $i=j$ this assertion directly follows from Lemma 2.2 in Berkes et al. (2013). For $i \neq j$, one can use similar arguments as in the proof of the latter Lemma. More precisely, assume without loss of generality that $i=1, j=2$ and show that

$$
\begin{aligned}
\left|\Sigma_{12}\right| & <\infty, \\
\left|\left(\Sigma_{m}\right)_{12}\right| & <\infty, \quad m \geq 1, \\
\left(\Sigma_{m}\right)_{12} & \rightarrow \Sigma_{12}, \quad \text { as } m \rightarrow \infty .
\end{aligned}
$$


From the estimate

$$
\left|\Sigma_{12}\right| \leq\left|\int_{T} \int_{T} \mathbb{E}\left[\eta_{0}^{(1)}(s) \eta_{0}^{(2)}(t)\right] \zeta(s) \zeta(t) d s d t\right|+2\left|\sum_{h=1}^{\infty} \int_{T} \int_{T} \mathbb{E}\left[\eta_{0}^{(1)}(s) \eta_{h}^{(2)}(t)\right] \zeta(s) \zeta(t) d s d t\right|
$$

B.13 follows if each of the terms above is finite. For the first term, we apply the Cauchy-Schwarz inequality to obtain by (A3)

$$
\begin{aligned}
\left|\int_{T} \int_{T} \mathbb{E}\left[\eta_{0}^{(1)}(s) \eta_{0}^{(2)}(t)\right] \zeta(s) \zeta(t) d s d t\right| & \leq\|\zeta\|^{2}\left(\int_{T} \int_{T} \mathbb{E}\left[\eta_{0}^{(1)}(s) \eta_{0}^{(2)}(t)\right]^{2} d s d t\right)^{1 / 2} \\
& \leq\|\zeta\|^{2}\left(\int_{T} \int_{T} \mathbb{E}\left[\eta_{0}^{(1)}(s)^{2}\right] \mathbb{E}\left[\eta_{0}^{(2)}(t)^{2}\right] d s d t\right)^{1 / 2} \\
& =\|\zeta\|^{2}\left(\mathbb{E}\left\|\eta_{0}^{(1)}\right\|^{2} \mathbb{E}\left\|\eta_{0}^{(2)}\right\|^{2}\right)^{1 / 2}<\infty .
\end{aligned}
$$

We proceed with the second term in $(\bar{B} .16)$ and drop the constant 2 . For any $i \geq 1, \mathbb{E}\left[\eta_{0}^{(1)} \eta_{i, i}^{(2)}\right]=$ $\mathbb{E}\left[\eta_{0}^{(1)}\right] \mathbb{E}\left[\eta_{i, i}^{(2)}\right]=0$ since $\eta_{0}^{(1)}$ and $\eta_{i, i}^{(2)}$ are independent. Applying the triangle inequality and the Cauchy-Schwarz inequality yields

$$
\begin{aligned}
& \left|\sum_{h=1}^{\infty} \int_{T} \int_{T} \mathbb{E}\left[\eta_{0}^{(1)}(s) \eta_{h}^{(2)}(t)\right] \zeta(s) \zeta(t) d s d t\right| \\
& \leq \sum_{h=1}^{\infty}\|\zeta\|^{2}\left(\int_{T} \int_{T} \mathbb{E}\left[\eta_{0}^{(1)}(s) \eta_{h}^{(2)}(t)\right]^{2} d s d t\right)^{1 / 2} \\
& =\sum_{h=1}^{\infty}\|\zeta\|^{2}\left(\int_{T} \int_{T} \mathbb{E}\left[\eta_{0}^{(1)}(s)\left(\eta_{h}^{(2)}(t)-\eta_{h, h}^{(2)}(t)\right)\right]^{2} d s d t\right)^{1 / 2} \\
& \leq \sum_{h=1}^{\infty}\|\zeta\|^{2}\left(\int_{T} \int_{T} \mathbb{E}\left[\eta_{0}^{(1)}(s)^{2}\right] \mathbb{E}\left[\left(\eta_{h}^{(2)}(t)-\eta_{h, h}^{(2)}(t)\right)^{2}\right] d s d t\right)^{1 / 2} \\
& =\|\zeta\|^{2} \mathbb{E}\left[\left\|\eta_{0}^{(1)}\right\|^{2}\right]^{1 / 2} \sum_{h=1}^{\infty} \mathbb{E}\left[\left\|\eta_{0}^{(2)}-\eta_{0, h}^{(2)}\right\|^{2}\right]^{1 / 2} .
\end{aligned}
$$

Due to condition (A3) we have $\mathbb{E}\left[\left\|\eta_{0}^{(1)}(s)\right\|^{2}\right]^{1 / 2}<\infty$ and by an application of the Hölder inequality we get

$$
\sum_{h=1}^{\infty} \mathbb{E}\left[\left\|\eta_{h}^{(2)}-\eta_{h, h}^{(2)}\right\|^{2}\right]^{1 / 2} \leq \sum_{h=1}^{\infty} \mathbb{E}\left[\left\|\eta_{0}^{(2)}-\eta_{0, h}^{(2)}\right\|^{2+\psi}\right]^{1 /(2+\psi)}
$$

which is finite by (A4). This completes the proof of (B.13).

For $\left(\Sigma_{m}\right)_{12}$, we proceed similarly. We have $\mathbb{E}\left[\eta_{0}^{(1)}(s) \eta_{0}^{(2)}(t)\right]=\mathbb{E}\left[\eta_{0, m}^{(1)}(s) \eta_{0, m}^{(2)}(t)\right]$ and therefore

$$
\left|\int_{T} \int_{T} \mathbb{E}\left[\eta_{0, m}^{(1)}(s) \eta_{0, m}^{(2)}(t)\right] \zeta(s) \zeta(t) d s d t\right|<\infty
$$


Note that the vectors $\left(\eta_{0, m}^{(1)}, \eta_{h, m}^{(2)}\right)$ and $\left(\eta_{0}^{(1)}, \eta_{h, m}^{(2)}\right)$ have the same distribution for all $h=1, \ldots, m$. Furthermore, the vectors $\left(\eta_{h, m}^{(1)}, \eta_{h, h}^{(2)}\right)$ and $\left(\eta_{0}^{(1)}, \eta_{0, h}^{(2)}\right)$ have the same distribution for all $h=1, \ldots, m$ (this follows from the definition of all quantities involved and the i.i.d. structure of the $\varepsilon_{i, j, m}^{*}$ ). Thus, using similar arguments as in (B.17), we obtain

$$
\begin{aligned}
& \left|\sum_{h=1}^{m} \int_{T} \int_{T} \mathbb{E}\left[\eta_{0, m}^{(1)}(s) \eta_{h, m}^{(2)}(t)\right] \zeta(s) \zeta(t) d s d t\right| \leq\|\zeta\|^{2} \sum_{h=1}^{m}\left(\int_{T} \int_{T} \mathbb{E}\left[\eta_{0, m}^{(1)}(s) \eta_{h, m}^{(2)}(t)\right]^{2} d s d t\right)^{1 / 2} \\
= & \|\zeta\|^{2} \sum_{h=1}^{m}\left(\int_{T} \int_{T} \mathbb{E}\left[\eta_{0}^{(1)}(s) \eta_{h, m}^{(2)}(t)\right]^{2} d s d t\right)^{1 / 2} \leq\|\zeta\|^{2} \mathbb{E}\left[\left\|\eta_{0}^{(1)}\right\|^{2}\right]^{1 / 2} \sum_{h=1}^{m} \mathbb{E}\left[\left\|\eta_{h, m}^{(2)}-\eta_{h, h}^{(2)}\right\|^{2}\right]^{1 / 2} \\
\leq & \|\zeta\|^{2} \mathbb{E}\left[\left\|\eta_{0}^{(1)}\right\|^{2}\right]^{1 / 2} \sum_{h=1}^{\infty} \mathbb{E}\left[\left\|\eta_{0}^{(2)}-\eta_{0, h}^{(2)}\right\|^{2}\right]^{1 / 2}<\infty
\end{aligned}
$$

which proves B.14).

In order to establish (B.15), we begin by observing that

$$
\begin{aligned}
& \left|\sum_{h=0}^{\infty} \int_{T} \int_{T} \mathbb{E}\left[\eta_{0}^{(1)}(s) \eta_{h}^{(2)}(t)\right] \zeta(s) \zeta(t) d s d t-\sum_{h=0}^{m} \int_{T} \int_{T} \mathbb{E}\left[\eta_{0, m}^{(1)}(s) \eta_{h, m}^{(2)}(t)\right] \zeta(s) \zeta(t) d s d t\right| \\
\leq & \left|\sum_{h=1}^{m} \int_{T} \int_{T}\left\{\mathbb{E}\left[\eta_{0}^{(1)}(s) \eta_{h}^{(2)}(t)\right]-\mathbb{E}\left[\eta_{0, m}^{(1)}(s) \eta_{h, m}^{(2)}(t)\right]\right\} \zeta(s) \zeta(t) d s d t\right| \\
& +\sum_{h=m+1}^{\infty}\left|\int_{T} \int_{T} \mathbb{E}\left[\eta_{0}^{(1)}(s) \eta_{h}^{(2)}(t)\right] \zeta(s) \zeta(t) d s d t\right| \\
\leq & \sum_{h=1}^{m}\left|\int_{T} \int_{T} \mathbb{E}\left[\eta_{0}^{(1)}(s)\left\{\eta_{h}^{(2)}(t)-\eta_{h, m}^{(2)}(t)\right\}\right] \zeta(s) \zeta(t) d s d t\right| \\
& +\|\zeta\|^{2} \mathbb{E}\left[\left\|\eta_{0}^{(1)}\right\|^{2}\right]^{1 / 2} \sum_{h=m+1}^{\infty} \mathbb{E}\left[\left\|\eta_{0}^{(2)}-\eta_{0, h}^{(2)}\right\|^{2}\right]^{1 / 2},
\end{aligned}
$$

where the last inequality follows by similar arguments as (B.17). Now the second term converges to zero as $m \rightarrow \infty$ and for the first term we obtain

$$
\begin{aligned}
& \sum_{h=1}^{m}\left|\int_{T} \int_{T} \mathbb{E}\left[\eta_{0}^{(1)}(s)\left\{\eta_{h}^{(2)}(t)-\eta_{h, m}^{(2)}(t)\right\}\right] \zeta(s) \zeta(t) d s d t\right| \\
\leq & \|\zeta\|^{2} \sum_{h=1}^{m}\left(\int_{T} \int_{T} \mathbb{E}\left[\eta_{0}^{(1)}(s)\left\{\eta_{h}^{(2)}(t)-\eta_{h, m}^{(2)}(t)\right\}\right]^{2} d s d t\right)^{1 / 2} .
\end{aligned}
$$

Now by (A4) we have for any fixed $h$

$$
\begin{aligned}
\left(\int_{T} \int_{T} \mathbb{E}\left[\eta_{0}^{(1)}(s)\left\{\eta_{h}^{(2)}(t)-\eta_{h, m}^{(2)}(t)\right\}\right]^{2} d s d t\right)^{1 / 2} & \leq \mathbb{E}\left[\left\|\eta_{0}^{(1)}\right\|^{2}\right]^{1 / 2} \mathbb{E}\left[\left\|\eta_{h}^{(2)}-\eta_{h, m}^{(2)}\right\|^{2}\right]^{1 / 2} \\
& =\mathbb{E}\left[\left\|\eta_{0}^{(1)}\right\|^{2}\right]^{1 / 2} \mathbb{E}\left[\left\|\eta_{0}^{(2)}-\eta_{0, m}^{(2)}\right\|^{2}\right]^{1 / 2} \rightarrow 0
\end{aligned}
$$


Since also by similar arguments as in (B.17) and in the proof of (B.14

$$
\begin{aligned}
& \left(\int_{T} \int_{T} \mathbb{E}\left[\eta_{0}^{(1)}(s)\left\{\eta_{h}^{(2)}(t)-\eta_{h, m}^{(2)}(t)\right\}\right]^{2} d s d t\right)^{1 / 2} \\
\leq & 2\left\{\left(\int_{T} \int_{T} \mathbb{E}\left[\eta_{0}^{(1)}(s) \eta_{h}^{(2)}(t)\right]^{2} d s d t\right)^{1 / 2}+\left(\int_{T} \int_{T} \mathbb{E}\left[\eta_{0}^{(1)}(s) \eta_{h, m}^{(2)}(t)\right]^{2} d s d t\right)^{1 / 2}\right\} \\
\leq & 4 \mathbb{E}\left[\left\|\eta_{0}^{(1)}\right\|^{2}\right]^{1 / 2} \mathbb{E}\left[\left\|\eta_{0}^{(2)}-\eta_{0, h}^{(2)}\right\|^{2}\right]^{1 / 2}
\end{aligned}
$$

and since the right-hand side is summable over $h \geq 1$ it follows that

$$
\sum_{h=1}^{m}\left|\int_{T} \int_{T} \mathbb{E}\left[\eta_{0}^{(1)}(s)\left\{\eta_{h}^{(2)}(t)-\eta_{h, m}^{(2)}(t)\right\}\right] \zeta(s) \zeta(t) d s d t\right| \rightarrow 0, \quad m \rightarrow \infty
$$

by the dominated convergence theorem for series.

The assertion in B.15 follows and this also completes the proof of (i).

The claim in (ii) follows by a direct application of Lemma 2.1 in Berkes et al. (2013).

For a proof of claim (iii) note that for each fixed $m$ the sequence

$$
\left(\int_{T} \eta_{i, m}^{(1)}(t) \zeta(t) d t, \int_{T} \eta_{i, m}^{(2)}(t) \zeta(t) d t\right)_{i \in \mathbb{Z}}
$$

form a collection of stationary, $m$-dependent random vectors with finite variance. Now (iii) follows by a straightforward application of the Cramer-Wold device and the CLT for m-dependent random variables, see for instance Theorem 9.1 in DasGupta (2008).

\section{B.2.2 Proof of Proposition 3.1}

Step 1: Recall the definition of $\hat{f}$ in $(3.4)$. We begin by proving the following preliminary result

$$
\left\{\mathbb{G}_{N}(\theta)\right\}_{\theta \in[0,1]}:=\{\sqrt{N}(\hat{f}(\lfloor N \theta\rfloor)-d(\theta))\}_{\theta \in[0,1]} \rightsquigarrow\{\mathbb{G}(\theta)\}_{\theta \in[0,1]}
$$

in $\ell^{\infty}([0,1])$ as $N \rightarrow \infty$, where

$$
d(\theta):=\tilde{d}(\theta) \int_{T} \delta(t)^{2} d t, \quad \tilde{d}(\theta)=\theta(1-\theta) \begin{cases}\left(\theta_{0} / \theta\right)^{2}, & 1>\theta>\theta_{0} \\ \left(\left(1-\theta_{0}\right) /(1-\theta)\right)^{2}, & 0<\theta \leq \theta_{0}\end{cases}
$$

$\tilde{d}(0)=\tilde{d}(1)=0$, and the process $\mathbb{G}$ is a random element in $\ell^{\infty}([0,1])$ with a.s. continuous sample paths. To this end define for $k=1, \ldots, N-1$

$$
\begin{aligned}
& A_{N}(t, k):=\frac{1}{k} \sum_{j=1}^{k}\left(X_{j}(t)-\mathbb{E}\left[X_{j}(t)\right]\right)-\frac{1}{N-k} \sum_{j=k+1}^{N}\left(X_{j}(t)-\mathbb{E}\left[X_{j}(t)\right]\right) \\
& B_{N}(t, k):=\frac{1}{k} \sum_{j=1}^{k} \mathbb{E}\left[X_{j}(t)\right]-\frac{1}{N-k} \sum_{j=k+1}^{N} \mathbb{E}\left[X_{j}(t)\right]
\end{aligned}
$$


and let $A_{N}(t, N)=A_{N}(t, 0)=B_{N}(t, N)=B_{N}(t, 0) \equiv 0$. With those definitions we can write

$$
\begin{aligned}
\hat{f}(k) & =\int_{T}\left(A_{N}(t, k)+B_{N}(t, k)\right)^{2} d t \frac{k}{N}\left(1-\frac{k}{N}\right) \\
& =\left\{\int_{T} A_{N}(t, k)^{2} d t+2 \int_{T} A_{N}(t, k) B_{N}(t, k) d t+\int_{T} B_{N}(t, k)^{2} d t\right\} \frac{k}{N}\left(1-\frac{k}{N}\right) .
\end{aligned}
$$

From Theorem 1.1 in Berkes et al. (2013) it follows that

$$
\frac{k}{N}\left(1-\frac{k}{N}\right) \int_{T} A_{N}(t, k)^{2} d t=o_{\mathbb{P}}\left(N^{-1 / 2}\right)
$$

uniformly with respect to $k$. For $1 \leq k \leq k_{0}:=\left\lfloor N \theta_{0}\right\rfloor$, straightforward calculations yield

$$
\begin{aligned}
B_{N}(t, k) & =\frac{1}{k} \sum_{j=1}^{k} \mu(t)-\frac{1}{N-k} \sum_{j=k+1}^{k_{0}} \mu(t)-\frac{1}{N-k} \sum_{j=k_{0}+1}^{N}(\mu(t)+\delta(t)) \\
& =\mu(t)\left(1-\frac{k_{0}-k}{N-k}\right)-(\mu(t)+\delta(t)) \frac{N-k_{0}}{N-k} \\
& =\mu(t)\left(1-\frac{\theta_{0}-k / N}{1-k / N}\right)-(\mu(t)+\delta(t)) \frac{1-\theta_{0}}{1-k / N}+O\left(N^{-1}\right) \\
& =-\frac{1-\theta_{0}}{1-k / N} \delta(t)+O\left(N^{-1}\right)
\end{aligned}
$$

and in the case $N>k>k_{0}$ we have (again uniformly in $k$ )

$$
\begin{aligned}
B_{N}(t, k) & =\frac{1}{k} \sum_{j=1}^{k_{0}} \mu(t)+\frac{1}{k} \sum_{j=k_{0}+1}^{k}(\mu(t)+\delta(t))-\frac{1}{N-k} \sum_{j=k+1}^{N}(\mu(t)+\delta(t)) \\
& =\frac{\theta_{0}}{k / N} \mu(t)+\frac{k / N-\theta_{0}}{k / N}(\mu(t)+\delta(t))-\frac{1-k / N}{1-k / N}(\mu(t)+\delta(t))+O\left(N^{-1}\right) \\
& =-\frac{\theta_{0}}{k / N} \delta(t)+O\left(N^{-1}\right) .
\end{aligned}
$$

Hence we obtain

$$
\frac{k}{N}\left(1-\frac{k}{N}\right) \int_{T} B_{N}(t, k)^{2} d t=d(k / N)+O\left(N^{-1}\right),
$$

uniformly with respect to $k$ and

$$
\frac{k}{N}\left(1-\frac{k}{N}\right) \int_{T} A_{N}(t, k) B_{N}(t, k) d t=\int_{T} A_{N}(t, k) \delta(t) d t \tilde{d}(k / N)+o_{\mathbb{P}}\left(N^{-1 / 2}\right) .
$$

Therefore we obtain from (B.20), B.21), B.22), Lipschitz continuity of $\theta \mapsto d(\theta), \theta \mapsto \tilde{d}(\theta)$ and the line above

$$
\mathbb{G}_{N}(\theta)=2 \sqrt{N}\left\{\int_{T} A_{N}(t,\lfloor N \theta\rfloor) \delta(t) d t \tilde{d}(\theta)\right\}+o_{\mathbb{P}}(1)
$$


uniformly with respect to $\theta \in[0,1]$. In order to investigate the leading term on the right hand side observe for any $\theta \in[1 / N, 1)$ the representation

$$
\begin{aligned}
A_{N}(t,\lfloor\theta N\rfloor)= & \frac{1}{\lfloor\theta N\rfloor}\left(\sum_{j=1}^{\left\lfloor\left(\theta \wedge \theta_{0}\right) N\right\rfloor} \eta_{j}^{(1)}(t)+\mathbb{1}\left\{\theta_{0}<\theta\right\} \sum_{j=\left\lfloor\theta_{0} N\right\rfloor+1}^{\lfloor\theta N\rfloor} \eta_{j}^{(2)}(t)\right) \\
& -\frac{1}{N-\lfloor\theta N\rfloor}\left(\mathbb{1}\left\{\theta_{0} \geq \theta\right\} \sum_{j=\lfloor\theta N\rfloor+1}^{\left\lfloor\theta_{0} N\right\rfloor} \eta_{j}^{(1)}(t)+\sum_{j=\left\lfloor\left(\theta \vee \theta_{0}\right) N\right\rfloor+1}^{N} \eta_{j}^{(2)}(t)\right) \\
= & \frac{1}{\lfloor\theta N\rfloor} \sum_{j=1}^{\left\lfloor\left(\theta \wedge \theta_{0}\right) N\right\rfloor} \eta_{j}^{(1)}(t)-\frac{\mathbb{1}\left\{\theta_{0} \geq \theta\right\}}{N-\lfloor\theta N\rfloor} \sum_{j=\lfloor\theta N\rfloor+1}^{\left\lfloor\theta_{0} N\right\rfloor} \eta_{j}^{(1)}(t) \\
& +\frac{\mathbb{1}\left\{\theta_{0}<\theta\right\}}{\lfloor\theta N\rfloor} \sum_{j=\left\lfloor\theta_{0} N\right\rfloor+1}^{\lfloor\theta N\rfloor} \eta_{j}^{(2)}(t)-\frac{1}{N-\lfloor\theta N\rfloor} \sum_{j=\left\lfloor\left(\theta \vee \theta_{0}\right) N\right\rfloor+1}^{N} \eta_{j}^{(2)}(t),
\end{aligned}
$$

which yields

$$
\begin{aligned}
& 2 \sqrt{N} \int_{T} A_{N}(t,\lfloor N \theta\rfloor) \delta(t) d t \\
& =\frac{N}{\lfloor\theta N\rfloor} \tilde{\mathbb{Z}}_{N}^{(1)}\left(\theta \wedge \theta_{0}\right)-\mathbb{1}\left\{\theta_{0} \geq \theta\right\} \frac{N}{N-\lfloor\theta N\rfloor}\left(\tilde{\mathbb{Z}}_{N}^{(1)}\left(\theta_{0}\right)-\tilde{\mathbb{Z}}_{N}^{(1)}(\theta)\right) \\
& \quad+\mathbb{1}\left\{\theta_{0}<\theta\right\} \frac{N}{\lfloor\theta N\rfloor}\left(\tilde{\mathbb{Z}}_{N}^{(2)}(\theta)-\tilde{\mathbb{Z}}_{N}^{(2)}\left(\theta_{0}\right)\right)-\frac{N}{N-\lfloor\theta N\rfloor}\left(\tilde{\mathbb{Z}}_{N}^{(2)}(1)-\tilde{\mathbb{Z}}_{N}^{(2)}\left(\theta_{0} \vee \theta\right)\right),
\end{aligned}
$$

where

$$
\tilde{\mathbb{Z}}_{N}^{(i)}(\lambda)=\frac{1}{\sqrt{N}} \sum_{j=1}^{\lfloor\lambda N\rfloor} \int_{T} \eta_{j}^{(i)}(t) \delta(t) d t
$$

Finally, note that we have

$$
\sup _{\theta \in[1 / N, 1)}\left|\tilde{d}(\theta) \frac{N}{\lfloor\theta N\rfloor}-\frac{\tilde{d}(\theta)}{\theta}\right|=o(1), \quad \sup _{\theta \in[1 / N, 1)}\left|\tilde{d}(\theta) \frac{N}{N-\lfloor\theta N\rfloor}-\frac{\tilde{d}(\theta)}{1-\theta}\right|=o(1) .
$$

Hence Lemma B.1, Slutskys Lemma and the continuous mapping theorem yield

$$
2 \sqrt{N}\left\{\int_{T} A_{N}(t,\lfloor N \theta\rfloor) \delta(t) d t \tilde{d}(\theta)\right\}_{\theta \in[0,1]} \rightsquigarrow\{\mathbb{G}(\theta)\}_{\theta \in[0,1]} .
$$

Combing (B.25) with (B.23) gives us the weak convergence in (B.18).

Step 2: Given the weak convergence in (B.18) we are ready to prove (3.5). The proof will proceed in three steps. First, we show that $\hat{\theta}=\theta_{0}+o_{\mathbb{P}}(1)$. In the second step we show that

$$
\hat{\theta}=\theta_{0}+o_{\mathbb{P}}\left(N^{-1 / 4}\right) .
$$


In the final step we derive 3.5.

Observe that the function $\theta \mapsto d(\theta)$, defined in (B.19), is strictly increasing in $\left[0, \theta_{0}\right]$ and strictly decreasing in $\left(\theta_{0}, 1\right]$. Therefore, for any $\tilde{\delta}>0$, there is an $\varepsilon>0$ such that $\left|\theta-\theta_{0}\right|>\tilde{\delta}$ implies $d\left(\theta_{0}\right)-d(\theta)>\varepsilon$. Now let $\tilde{\delta}>0$ be arbitrary and assume $|\hat{\theta}-\theta|>\tilde{\delta}$. Using that $\hat{k}:=N \hat{\theta}$ is the maximizer of the function $k \mapsto f(k)$, the result from Step 1 and the previously mentioned monotonicity property, we obtain

$$
\begin{aligned}
0 & \geq \hat{f}\left(k_{0}\right)-\hat{f}(\hat{k})=\hat{f}\left(k_{0}\right)-d\left(\theta_{0}\right)-(\hat{f}(\hat{k})-d(\hat{\theta}))+d\left(\theta_{0}\right)-d(\hat{\theta}) \\
& =O_{\mathbb{P}}\left(N^{-1 / 2}\right)+d\left(\theta_{0}\right)-d(\hat{\theta})>O_{\mathbb{P}}\left(N^{-1 / 2}\right)+\varepsilon
\end{aligned}
$$

for some $\varepsilon>0$, where $k_{0}:=\left\lfloor N \theta_{0}\right\rfloor$. This means that

$$
\mathbb{P}\left(\left|\hat{\theta}-\theta_{0}\right|>\tilde{\delta}\right) \leq \mathbb{P}\left(O_{\mathbb{P}}\left(N^{-1 / 2}\right)<-\varepsilon\right) \rightarrow 0
$$

as $N \rightarrow \infty$ and therefore, $\hat{\theta}$ converges to the true change point $\theta_{0}$ in probability.

Next we show that $\left|\hat{\theta}-\theta_{0}\right|=O_{\mathbb{P}}\left(N^{-1 / 2}\right)$. Making a Taylor expansion of $d$ at the point $\theta_{0}$, we obtain, as $\theta \rightarrow \theta_{0}$,

$$
d(\theta)=d\left(\theta_{0}\right)+c\left(-\left(\theta-\theta_{0}\right) \mathbb{1}\left\{\theta>\theta_{0}\right\}+\left(\theta-\theta_{0}\right) \mathbb{1}\left\{\theta \leq \theta_{0}\right\}\right)+O\left(\left(\theta-\theta_{0}\right)^{2}\right)
$$

for some constant $c>0$. Therefore, as $\theta \rightarrow \theta_{0}$, we can find a constant $\tilde{\delta}>0$ such that

$$
d\left(\theta_{0}\right)-d(\theta) \geq \tilde{\delta}\left|\theta-\theta_{0}\right|+O\left(\left(\theta-\theta_{0}\right)^{2}\right) .
$$

Since $\hat{\theta}$ is a consistent estimator of $\theta_{0}$ (by the discussion in the previous paragraph), we can use this property and similar arguments as in (B.27) to obtain

$$
0 \leq \hat{f}(\hat{k})-\hat{f}\left(k_{0}\right)=O_{\mathbb{P}}\left(N^{-1 / 2}\right)+d(\hat{\theta})-d\left(\theta_{0}\right) \leq O_{\mathbb{P}}\left(N^{-1 / 2}\right)-\tilde{\delta}\left|\hat{\theta}-\theta_{0}\right|
$$

which means that $\left|\hat{\theta}-\theta_{0}\right|=O_{\mathbb{P}}\left(N^{-1 / 2}\right)$.

Thus, with probability converging to 1 , we have $\hat{\theta} \in \operatorname{argmax}_{\theta:\left|\theta-\theta_{0}\right| \leq N^{-1 / 4}} \hat{f}(\lfloor N \theta\rfloor)$. Since the process $\mathbb{G}_{N}$ in $(\mathrm{B} .18)$ is stochastically equicontinuous, we get

$$
\begin{aligned}
\left|\hat{f}(\hat{k})-\hat{f}\left(k_{0}\right)-\left(d(\hat{\theta})-d\left(\theta_{0}\right)\right)\right| & \leq \sup _{\theta:\left|\theta-\theta_{0}\right| \leq N^{-1 / 4}}\left|\hat{f}(\lfloor N \theta\rfloor)-\hat{f}\left(k_{0}\right)-\left(d(\theta)-d\left(\theta_{0}\right)\right)\right| \\
& \leq \sup _{\theta, \theta^{\prime}:\left|\theta-\theta^{\prime}\right| \leq N^{-1 / 4}} N^{-1 / 2}\left|\mathbb{G}_{N}(\theta)-\mathbb{G}_{N}\left(\theta^{\prime}\right)\right| \\
& =o_{\mathbb{P}}\left(N^{-1 / 2}\right) .
\end{aligned}
$$

Using this rate and the bound in (B.28) yields

$$
\begin{aligned}
0 \leq \hat{f}(\hat{k})-\hat{f}\left(k_{0}\right) & \leq d(\hat{\theta})-d\left(\theta_{0}\right)+\left|\hat{f}(\hat{k})-\hat{f}\left(k_{0}\right)-\left(d(\hat{\theta})-d\left(\theta_{0}\right)\right)\right| \\
& \leq d(\hat{\theta})-d\left(\theta_{0}\right)+o_{\mathbb{P}}\left(N^{-1 / 2}\right) \\
& \leq-\tilde{\delta}\left|\theta_{0}-\hat{\theta}\right|+o_{\mathbb{P}}\left(N^{-1 / 2}\right)
\end{aligned}
$$

which finally implies $\left|\hat{\theta}-\theta_{0}\right|=o_{\mathbb{P}}\left(N^{-1 / 2}\right)$. 


\section{B.2.3 Proof of Theorem 3.1}

We begin by stating some useful technical results and notations. Define

$$
\tilde{S}_{N}^{(k)}(t, \lambda)=\frac{1}{N} \sum_{j=1}^{\lfloor\lambda N\rfloor} \eta_{j}^{(k)}(t), \quad k=1,2
$$

where $\eta_{j}^{(1)}:=f_{1}\left(\varepsilon_{j}, \varepsilon_{j-1, \ldots}\right), \eta_{j}^{(2)}:=f_{2}\left(\varepsilon_{j}, \varepsilon_{j-1, \ldots}\right)$ for $j \in \mathbb{Z}$. Since $f_{1}, f_{2}$ satisfy assumptions (A3), (A4), it follows from Theorem 1.1 in Berkes et al. (2013) that there exist random elements in $\mathcal{G}$ (recall the beginning of Section B), say $\Gamma_{N}^{(i)}$, with

$$
\sup _{\lambda \in[0,1]} \int_{T}\left(\sqrt{N} \tilde{S}_{N}^{(i)}(t, \lambda)-\Gamma_{N}^{(i)}(t, \lambda)\right)^{2} d t=o_{P}(1), \quad i=1,2
$$

where each $\Gamma_{N}^{(i)}$ satisfies the analogue of $(\mathrm{B} .3)-(\mathrm{B} .8)$ with covariance kernels corresponding to $\eta_{i}^{(1)}$ and $\eta_{i}^{(2)}$, respectively.

First consider the case $\int \delta^{2}(t) d t \neq 0$. Recalling that $\hat{\theta}=\theta_{0}+o_{\mathbb{P}}\left(N^{-1 / 2}\right)$ by Proposition 3.1 we proceed in several steps. First, we show that for the process

$$
\mathbb{Z}_{N}(\lambda, \theta)=\sqrt{N} \int_{T}\left(D_{N}^{c p}(t, \lambda, \theta)^{2}-\lambda^{2} \delta(t)^{2}\right) d t
$$

we have

$$
\left\{\mathbb{Z}_{N}\left(\lambda, \theta_{0}\right)\right\}_{\lambda \in[0,1]} \rightsquigarrow\left\{\lambda \tau_{\delta, \theta_{0}} \mathbb{B}(\lambda)\right\}_{\lambda \in[0,1]}
$$

in $\ell^{\infty}([0,1])$, where $\{\mathbb{B}(\lambda)\}_{\lambda \in[0,1]}$ is a Brownian motion and $\tau_{\delta, \theta_{0}}$ is a parameter depending on the covariance structure of $\left\{\left(\eta_{j}^{(1)}\right\},\left\{\eta_{j}^{(2)}\right)\right\}_{j \in \mathbb{Z}}$ and the true change point location $\theta_{0}$. Second we prove

$$
\sup _{\lambda \in[0,1]}\left|\mathbb{Z}_{N}\left(\lambda, \theta_{0}\right)-\mathbb{Z}_{N}(\lambda, \hat{\theta})\right|=o_{\mathbb{P}}(1)
$$

where $\hat{\theta}$ is the estimator of $\theta_{0}$ defined in $(3.3)$. Finally we can again use the same arguments as in the proof of Theorem 2.1 to obtain the assertion.

Next, consider the case $\int_{T} \delta^{2}(t) d t=0$. It suffices to show that $\hat{\mathbb{D}}_{N}^{c p}=o_{\mathbb{P}}(1), \hat{\mathbb{V}}_{N}^{c p}=o_{\mathbb{P}}(1)$. To this end define the partial sum process

$$
W_{N}(t, \lambda):=\frac{1}{N}\left\{\sum_{i=1}^{\left\lfloor N\left(\lambda \wedge \theta_{0}\right)\right\rfloor} \eta_{i}^{(1)}(t)+\sum_{i=\left\lfloor N \theta_{0}\right\rfloor+1}^{\left\lfloor N\left(\lambda \vee \theta_{0}\right)\right\rfloor} \eta_{i}^{(2)}(t)\right\}
$$

and observe that by (B.30) and some elementary computations we have

$$
\sup _{\lambda \in[0,1]} \int_{T} W_{N}^{2}(t, \lambda) d t=o_{\mathbb{P}}(1)
$$


Next, observing that

$$
\begin{aligned}
& D_{N}^{c p}(t, \lambda, \theta) \\
= & \frac{N}{\lfloor N \theta\rfloor} W_{N}\left(t, \frac{\lfloor\lambda\lfloor N \theta\rfloor\rfloor}{N}\right)-\frac{N}{N-\lfloor N \theta\rfloor}\left\{W_{N}\left(t, \frac{\lfloor N \theta\rfloor+\lfloor\lambda(N-\lfloor N \theta\rfloor)\rfloor}{N}\right)-W_{N}\left(t, \frac{\lfloor N \theta\rfloor}{N}\right)\right\},
\end{aligned}
$$

some elementary calculations taking into account that by definition $\hat{\theta} \in[\varepsilon, 1-\varepsilon]$ show that

$$
\hat{\mathbb{V}}_{N}^{c p} \leq 4 \sup _{\lambda \in[0,1], \theta \in[\varepsilon, 1-\varepsilon]} \int_{T}\left\{D_{N}^{c p}(t, \lambda, \theta)\right\}^{2} d t \lesssim \frac{1}{\varepsilon^{2}} \sup _{\lambda \in[0,1]} \int_{T} W_{N}^{2}(t, \lambda) d t=o_{\mathbb{P}}(1) .
$$

Similar but simpler arguments show that $\hat{\mathbb{D}}_{N}^{c p}=o_{\mathbb{P}}(1)$ and this completes the proof in the case $\int_{T} \delta^{2}(t) d t=0$

Proof of B.31. Define the processes

$$
\begin{aligned}
& S_{N}^{(1)}(t, \lambda, \theta)=\frac{1}{\lfloor\theta N\rfloor} \sum_{j=1}^{\lfloor\lambda\lfloor\theta N\rfloor\rfloor}\left(X_{j}(t)-\mu(t)\right) \\
& S_{N}^{(2)}(t, \lambda, \theta)=\frac{1}{N-\lfloor\theta N\rfloor} \sum_{j=\lfloor\theta N\rfloor+1}^{\lfloor\theta N\rfloor+\lfloor\lambda(N-\lfloor\theta N\rfloor)\rfloor}\left(X_{j}(t)-\mu(t)-\delta(t)\right)
\end{aligned}
$$

and similar to the calculations in Section B.1.1 we can write

$$
\begin{aligned}
\mathbb{Z}_{N}(\lambda, \theta)= & \sqrt{N} \int_{T}\left(D_{N}^{c p}(t, \lambda, \theta)-\lambda \delta(t)\right)^{2} d t+2 \sqrt{N} \int_{T} \lambda \delta(t)\left(D_{N}^{c p}(t, \lambda, \theta)-\lambda \delta(t)\right) d t \\
= & \sqrt{N} \int_{T}\left(S_{N}^{(1)}(t, \lambda, \theta)-S_{N}^{(2)}(t, \lambda, \theta)\right)^{2} d t \\
& +2 \sqrt{N} \int_{T} \lambda \delta(t)\left(S_{N}^{(1)}(t, \lambda, \theta)-S_{N}^{(2)}(t, \lambda, \theta)\right) d t+o_{\mathbb{P}}(1)
\end{aligned}
$$

uniformly in $\lambda \in[0,1], \theta \in(\varepsilon, 1-\varepsilon)$ for any $\varepsilon>0$. For $\theta=\theta_{0}$, the first term at the end of the calculation above converges to zero (as in the two sample case) and the second term can be rewritten such that Lemma B.1 can be applied

$$
\begin{aligned}
\mathbb{Z}_{N}\left(\lambda, \theta_{0}\right) & =2 \sqrt{N} \int_{T} \lambda \delta(t)\left(S_{N}^{(1)}\left(t, \lambda, \theta_{0}\right)-S_{N}^{(2)}\left(t, \lambda, \theta_{0}\right)\right) d t+o_{\mathbb{P}}(1) \\
& =\mathbb{Z}_{N}^{(1)}\left(\lambda, \theta_{0}\right)-\mathbb{Z}_{N}^{(2)}\left(\lambda, \theta_{0}\right)+o_{\mathbb{P}}(1),
\end{aligned}
$$

where

$$
\mathbb{Z}_{N}^{(i)}(\lambda, \theta)=2 \sqrt{N} \int_{T} \lambda \delta(t) S_{N}^{(i)}(t, \lambda, \theta) d t, i=1,2 .
$$


We can rewrite the processes in (B.34) as

$$
\begin{aligned}
\mathbb{Z}_{N}^{(1)}\left(\lambda, \theta_{0}\right)-\mathbb{Z}_{N}^{(2)}\left(\lambda, \theta_{0}\right)= & 2 \frac{N}{\left\lfloor\theta_{0} N\right\rfloor} \lambda \frac{1}{\sqrt{N}} \sum_{j=1}^{\left\lfloor\lambda\left\lfloor\theta_{0} N\right\rfloor\right\rfloor} \int_{T} \eta_{j}^{(1)}(t) \delta(t) d t \\
& -2 \frac{N}{N-\left\lfloor\theta_{0} N\right\rfloor} \lambda \frac{1}{\sqrt{N}} \sum_{j=\left\lfloor\theta_{0} N\right\rfloor+1}^{\left\lfloor\theta_{0} N\right\rfloor+\left\lfloor\lambda\left(N-\left\lfloor\theta_{0} N\right\rfloor\right)\right\rfloor} \int_{T} \eta_{j}^{(2)}(t) \delta(t) d t \\
= & \frac{2 \lambda}{\theta_{0}} \tilde{\mathbb{Z}}_{N}^{(1)}\left(\left\lfloor\lambda\left\lfloor\theta_{0} N\right\rfloor\right\rfloor / N\right) \\
& -\frac{2 \lambda}{1-\theta_{0}}\left\{\tilde{\mathbb{Z}}_{N}^{(2)}\left(\left(\left\lfloor\theta_{0} N\right\rfloor+\left\lfloor\lambda\left(N-\left\lfloor\theta_{0} N\right\rfloor\right)\right\rfloor\right) / N\right)-\tilde{\mathbb{Z}}_{N}^{(2)}\left(\theta_{0}\right)\right\}+o_{\mathbb{P}}(1),
\end{aligned}
$$

where the remainder is uniform in $\lambda \in[0,1]$ and the processes $\tilde{\mathbb{Z}}_{N}^{(1)}$ and $\tilde{\mathbb{Z}}_{N}^{(2)}$ are defined in (B.24). An application of Lemma B.1 with $\zeta=\delta$, asymptotic equicontinuity of the sample paths of $\tilde{\mathbb{Z}}_{N}^{(i)}$, and the continuous mapping theorem yield the assertion in B.31. To see that the limit has the right structure, observe that

$$
\mathbb{Z}_{N}^{(1)}\left(\lambda, \theta_{0}\right)-\mathbb{Z}_{N}^{(2)}\left(\lambda, \theta_{0}\right) \rightsquigarrow \frac{2 \lambda}{\theta_{0}} \tilde{\mathbb{Z}}^{(1)}\left(\lambda \theta_{0}\right)-\frac{2 \lambda}{1-\theta_{0}}\left\{\tilde{\mathbb{Z}}^{(2)}\left(\theta_{0}+\lambda\left(1-\theta_{0}\right)\right)-\tilde{\mathbb{Z}}^{(2)}\left(\theta_{0}\right)\right\}
$$

where

$$
\tilde{\mathbb{Z}}^{(1)}=\tilde{\Sigma}_{11} \mathbb{B}_{1}+\tilde{\Sigma}_{12} \mathbb{B}_{2} ; \quad \tilde{\mathbb{Z}}^{(2)}=\tilde{\Sigma}_{21} \mathbb{B}_{1}+\tilde{\Sigma}_{22} \mathbb{B}_{2}
$$

and $\tilde{\Sigma}_{i j}$ denotes the $i j$-th entry of $\Sigma^{1 / 2}$. By straightforward calculations one obtains

$$
\begin{aligned}
\operatorname{Cov}\left(\tilde{\mathbb{Z}}^{(1)}\left(\lambda_{1} \theta_{0}\right), \tilde{\mathbb{Z}}^{(2)}\left(\theta_{0}+\lambda_{2}\left(1-\theta_{0}\right)\right)-\tilde{\mathbb{Z}}^{(2)}\left(\theta_{0}\right)\right) & =0, \\
\operatorname{Cov}\left(\tilde{\mathbb{Z}}^{(1)}\left(\lambda_{1} \theta_{0}\right), \tilde{\mathbb{Z}}^{(1)}\left(\lambda_{2} \theta_{0}\right)\right) & =\left(\lambda_{1} \wedge \lambda_{2}\right) \theta_{0}\left(\tilde{\Sigma}_{11}^{2}+\tilde{\Sigma}_{12}^{2}\right)=\left(\lambda_{1} \wedge \lambda_{2}\right) \theta_{0} \Sigma_{11}
\end{aligned}
$$

where $\Sigma$ is defined in the statement of Lemma B.1 and the last equation follows from the fact that $\tilde{\Sigma}$ is symmetric and that $\tilde{\Sigma} \tilde{\Sigma}=\Sigma$. Furthermore, $\tilde{\mathbb{Z}}^{(2)}\left(\theta_{0}+\lambda\left(1-\theta_{0}\right)\right)-\tilde{\mathbb{Z}}^{(2)}\left(\theta_{0}\right)$ has the same distribution as $\tilde{\mathbb{Z}}^{(2)}\left(\lambda\left(1-\theta_{0}\right)\right)$ and

$$
\operatorname{Cov}\left(\tilde{\mathbb{Z}}^{(2)}\left(\lambda_{1}\left(1-\theta_{0}\right)\right), \tilde{\mathbb{Z}}^{(2)}\left(\lambda_{2}\left(1-\theta_{0}\right)\right)\right)=\left(\lambda_{1} \wedge \lambda_{2}\right)\left(1-\theta_{0}\right)\left(\tilde{\Sigma}_{21}^{2}+\tilde{\Sigma}_{22}^{2}\right)=\left(\lambda_{1} \wedge \lambda_{2}\right)\left(1-\theta_{0}\right) \Sigma_{22} .
$$

Combining the calculations above, we can conclude that the limit process in $(\mathrm{B} .36)$ is of the form as claimed in (B.31).

Proof of B.32. For $\theta=\hat{\theta}$, we show that the first term at the end of the calculation in B.33 vanishes by proving

$$
N^{1 / 4} \sup _{\lambda \in[0,1]}\left\|S_{N}^{(i)}(\cdot, \lambda, \hat{\theta})\right\|=o_{\mathbb{P}}(1)
$$


for $i=1,2$. Since both cases are similar we only consider the case $i=1$. Write

$$
N^{1 / 4} S_{N}^{(1)}(t, \lambda, \hat{\theta})=\frac{1}{N^{1 / 4}} \frac{N}{\lfloor\hat{\theta} N\rfloor}\left(Q_{N}^{(1)}(t, \lambda, \hat{\theta})+Q_{N}^{(2)}(t, \lambda, \hat{\theta})\right)
$$

where

$$
\begin{aligned}
& Q_{N}^{(1)}(t, \lambda, \hat{\theta})=\frac{1}{\sqrt{N}} \sum_{j=1}^{\lfloor\lambda\lfloor\hat{\theta} N\rfloor\rfloor \wedge\left\lfloor\theta_{0} N\right\rfloor}\left(X_{j}(t)-\mu(t)\right)=\frac{1}{\sqrt{N}} \sum_{j=1}^{\lfloor\lambda\lfloor\hat{\theta} N\rfloor\rfloor \wedge\left\lfloor\theta_{0} N\right\rfloor} \eta_{j}^{(1)}(t) \\
& Q_{N}^{(2)}(t, \lambda, \hat{\theta})=\frac{1}{\sqrt{N}} \sum_{j=\lfloor\lambda\lfloor\hat{\theta} N\rfloor\rfloor \wedge\left\lfloor\theta_{0} N\right\rfloor+1}^{\lfloor\lambda\lfloor\hat{\theta} N\rfloor\rfloor}\left(X_{j}(t)-\mu(t)\right) .
\end{aligned}
$$

We have by (B.30) and the properties of $\Gamma_{N}^{(i)}$

$$
\begin{aligned}
& \lim _{p \rightarrow \infty} \limsup _{N \rightarrow \infty} \mathbb{P}\left(\sup _{\lambda \in[0,1]}\left\|Q_{N}^{(1)}(\cdot, \lambda, \hat{\theta})\right\|>p\right) \\
\leq & \lim _{p \rightarrow \infty} \limsup _{N \rightarrow \infty} \mathbb{P}\left(\sup _{\lambda \in[0,1]} \sqrt{N}\left\|\tilde{S}_{N}^{(1)}(\cdot, \lambda)\right\|>p\right) \\
= & \lim \limsup _{p \rightarrow \infty} \mathbb{P}\left(\sup _{\lambda \in[0,1]} \int_{T}\left\{\Gamma_{N}^{(1)}(t, \lambda)\right\}^{2} d t>p^{2}\right)+o(1)=0
\end{aligned}
$$

where $\tilde{S}_{N}^{(1)}$ is defined in $(\overline{B .29})$. Therefore $\sup _{\lambda \in[0,1]}\left\|Q_{N}^{(1)}(\cdot, \lambda, \hat{\theta})\right\|=O_{\mathbb{P}}(1)$. The second term in (B.39) is zero if $\lfloor\lambda\lfloor\hat{\theta} N\rfloor\rfloor \leq\left\lfloor\theta_{0} N\right\rfloor$ or, if $\lfloor\lambda\lfloor\hat{\theta} N\rfloor\rfloor>\left\lfloor\theta_{0} N\right\rfloor$, we can write

$$
\sup _{\lambda \in[0,1]}\left\|Q_{N}^{(2)}(\cdot, \lambda, \hat{\theta})\right\|=\sup _{\lambda \in[0,1]}\left\|\frac{1}{\sqrt{N}} \sum_{j=\left\lfloor\theta_{0} N\right\rfloor+1}^{\lfloor\lambda\lfloor\hat{\theta} N\rfloor\rfloor}\left(\eta_{j}^{(2)}+\delta\right)\right\| .
$$

The number of terms in the sum above is bounded by the distance between $\theta_{0}$ and $\hat{\theta}$ in the sense that

$$
\begin{aligned}
& \sup _{\lambda \in[0,1]}\left\|\frac{1}{\sqrt{N}} \sum_{j=\left\lfloor\theta_{0} N\right\rfloor+1}^{\lfloor\lambda \mid \hat{\theta} N\rfloor\rfloor}\left(\eta_{j}^{(2)}+\delta\right)\right\| \\
\lesssim & \sup _{\substack{\nu, \lambda \in[0,1]: \\
|\nu-\lambda| \leq\left|\theta_{0}-\hat{\theta}\right|}} \sqrt{N}\left\|\tilde{S}_{N}^{(2)}(\cdot, \nu)-\tilde{S}_{N}^{(2)}(\cdot, \lambda)\right\|+\frac{\lfloor\hat{\theta} N\rfloor-\left\lfloor\theta_{0} N\right\rfloor}{\sqrt{N}}\|\delta\| \\
= & \sup _{\substack{\nu, \lambda \in[0,1]: \\
|\nu-\lambda| \leq\left|\theta_{0}-\hat{\theta}\right|}} \sqrt{\int_{T}\left\{\Gamma_{N}^{(2)}(t, \nu)-\Gamma_{N}^{(2)}(t, \lambda)\right\}^{2} d t}+\frac{\lfloor\hat{\theta} N\rfloor-\left\lfloor\theta_{0} N\right\rfloor}{\sqrt{N}}\|\delta\|+o_{\mathbb{P}}(1),
\end{aligned}
$$

where the last equality follows by (B.30) and the definition of $\|\cdot\|$. The first term in (B.40) converges to zero in probability since $(\mathrm{B} .8)$ holds with $\Gamma_{N}^{(2)}$ instead of $\Gamma$ and since by (3.5) 
$\left|\theta_{0}-\hat{\theta}\right|=o_{\mathbb{P}}(1 / \sqrt{N})$. The latter also implies that the second term converges to zero in probability. Therefore we have $\sup _{\lambda \in[0,1]}\left\|Q_{N}^{(2)}(\cdot, \lambda, \hat{\theta})\right\|=o_{\mathbb{P}}(1)$. Recalling (B.38), we conclude that (B.37) holds in the case $i=1$ and similar arguments yield the statement for $i=2$. This means that we can continue the calculations in (B.33) for $\theta=\hat{\theta}$ and obtain

$$
\begin{aligned}
& \sqrt{N} \int_{T}\left(S_{N}^{(1)}(t, \lambda, \hat{\theta})-S_{N}^{(2)}(t, \lambda, \hat{\theta})\right)^{2} d t+2 \sqrt{N} \int_{T} \lambda \delta(t)\left(S_{N}^{(1)}(t, \lambda, \hat{\theta})-S_{N}^{(2)}(t, \lambda, \hat{\theta})\right) d t \\
& =2 \sqrt{N} \int_{T} \lambda D^{c p}(t)\left(S_{N}^{(1)}(t, \lambda, \hat{\theta})-S_{N}^{(2)}(t, \lambda, \hat{\theta})\right) d t+o_{\mathbb{P}}(1) \\
& =\mathbb{Z}_{N}^{(1)}(\lambda, \hat{\theta})-\mathbb{Z}_{N}^{(2)}(\lambda, \hat{\theta})+o_{\mathbb{P}}(1),
\end{aligned}
$$

where $\mathbb{Z}_{N}^{(i)}$, for $i=1,2$, is defined by $\mathrm{B} .35$. In order to prove $\mathrm{B} .32$ it consequently remains to show

$$
\sup _{\lambda \in[0,1]}\left|\mathbb{Z}_{N}^{(i)}(\lambda, \hat{\theta})-\mathbb{Z}_{N}^{(i)}\left(\lambda, \theta_{0}\right)\right|=o_{\mathbb{P}}(1)
$$

for $i=1,2$. For that purpose we write

$$
\begin{aligned}
& \sup _{\lambda \in[0,1]}\left|\mathbb{Z}_{N}^{(1)}(\lambda, \hat{\theta})-\mathbb{Z}_{N}^{(1)}\left(\lambda, \theta_{0}\right)\right| \\
& =\sup _{\lambda \in[0,1]} \mid 2 \sqrt{N} \int_{T} \lambda \delta(t)\left\{\left(\frac{1}{\lfloor\hat{\theta} N\rfloor}-\frac{1}{\left\lfloor\theta_{0} N\right\rfloor}\right) \sum_{j=1}^{\left\lfloor\lambda\left\lfloor\left(\hat{\theta} \wedge \theta_{0}\right) N\right\rfloor\right\rfloor}\left(X_{j}(t)-\mu(t)\right)\right. \\
& \left.+\left(\mathbb{1}\left\{\hat{\theta} \geq \theta_{0}\right\}-\mathbb{1}\left\{\hat{\theta}<\theta_{0}\right\}\right) \frac{1}{\left\lfloor\left(\hat{\theta} \vee \theta_{0}\right) N\right\rfloor} \sum_{j=\left\lfloor\lambda\left\lfloor\left(\hat{\theta} \wedge \theta_{0}\right) N\right\rfloor\right\rfloor+1}^{\left\lfloor\lambda\left\lfloor\left(\hat{\theta} \vee \theta_{0}\right) N\right\rfloor\right\rfloor}\left(X_{j}(t)-\mu(t)\right)\right\} d t \mid \\
& \leq 2\|\delta\| \lambda\left\{\frac{N}{\left\lfloor\theta_{0} N\right\rfloor} \frac{\left\lfloor\left\lfloor\theta_{0} N\right\rfloor-\lfloor\hat{\theta} N\rfloor \mid\right.}{\lfloor\hat{\theta} N\rfloor} \sup _{\lambda \in[0,1]}\left\|\frac{1}{\sqrt{N}} \sum_{j=1}^{\left\lfloor\lambda\left\lfloor\left(\hat{\theta} \wedge \theta_{0}\right) N\right\rfloor\right\rfloor}\left(X_{j}-\mu\right)\right\|\right. \\
& +\frac{N}{\left\lfloor\left(\hat{\theta} \vee \theta_{0}\right) N\right\rfloor} \sup _{\lambda \in[0,1]}\left\|\frac{1}{\sqrt{N}} \sum_{j=\left\lfloor\lambda\left\lfloor\left(\hat{\theta} \wedge \theta_{0}\right) N\right\rfloor\right\rfloor+1}^{\left\lfloor\lambda\left\lfloor\left(\hat{\theta} \vee \theta_{0}\right) N\right\rfloor\right\rfloor}\left(X_{j}-\mathbb{E}\left[X_{j}\right]\right)\right\| \\
& \left.+\frac{N}{\left\lfloor\left(\hat{\theta} \vee \theta_{0}\right) N\right\rfloor} \sup _{\lambda \in[0,1]} \frac{1}{\sqrt{N}} \sum_{j=\left\lfloor\lambda\left\lfloor\left(\hat{\theta} \wedge \theta_{0}\right) N\right\rfloor\right\rfloor+1}^{\left\lfloor\lambda\left\lfloor\left(\hat{\theta} \vee \theta_{0}\right) N\right\rfloor\right\rfloor}\|\delta\|\right\} \\
& \lesssim \frac{\left|\theta_{0}-\hat{\theta}\right|}{\theta_{0} \hat{\theta}} \sup _{\lambda \in[0,1]} \sqrt{N}\left\|\tilde{S}_{N}^{(1)}(\cdot, \lambda)\right\| \\
& +\frac{1}{\hat{\theta} \vee \theta_{0}}\left(\sum_{i=1}^{2} \sup _{\substack{\nu, \lambda \in[0,1]: \\
|\nu-\lambda| \leq\left|\theta_{0}-\hat{\theta}\right|}} \sqrt{N}\left\|\tilde{S}_{N}^{(i)}(\cdot, \nu)-\tilde{S}_{N}^{(i)}(\cdot, \lambda)\right\|+\frac{\lfloor\hat{\theta} N\rfloor-\left\lfloor\theta_{0} N\right\rfloor \mid}{\sqrt{N}}\|\delta\|\right)+o(1) \\
& =o_{\mathbb{P}}(1) .
\end{aligned}
$$


The last equality holds since $\left|\theta_{0}-\hat{\theta}\right|=o_{\mathbb{P}}(1 / \sqrt{N}), \sup _{\lambda \in[0,1]} \sqrt{N}\left\|\tilde{S}_{N}^{(1)}(\cdot, \lambda)\right\|=O_{\mathbb{P}}(1)$ and since

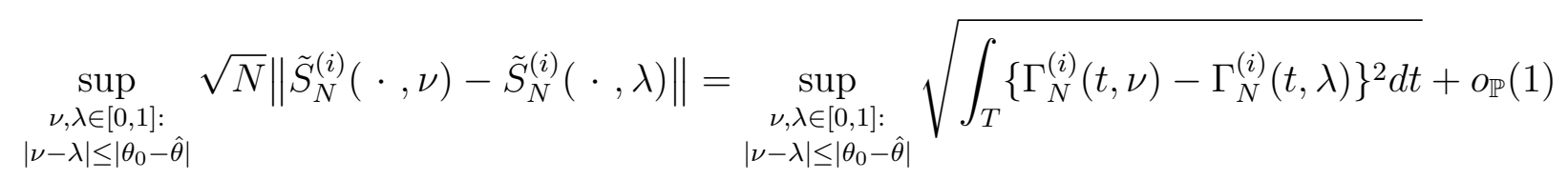

which is $o_{\mathbb{P}}(1)$ by similar arguments as given right after B.40. Similar arguments prove

$$
\sup _{\lambda \in[0,1]}\left|\mathbb{Z}_{N}^{(2)}(\lambda, \hat{\theta})-\mathbb{Z}_{N}^{(2)}\left(\lambda, \theta_{0}\right)\right|=o_{\mathbb{P}}(1)
$$

which finally implies B.32.

\section{B.3 Proof of Remark 3.2}

The proof is based on the following generalization of Lemma B.1. Assume model (3.11) and (m1). For an arbitrary finite collection of functions $\zeta_{1}, \ldots, \zeta_{M}$ in $L^{2}(T)$ consider the processes

$$
Z_{N}^{(j, m)}(\lambda):=\frac{1}{\sqrt{N_{j}}} \int_{T} \zeta_{m}(t) \tilde{S}_{N, j}(t, \lambda) d t, \quad j=1, \ldots, K, m=1, \ldots, M
$$

where

$$
\tilde{S}_{N, j}(t, \lambda)=\frac{1}{N_{j}} \sum_{i=1}^{\left\lfloor\lambda N_{j}\right\rfloor}\left(X_{\left\lfloor N \theta_{j-1}\right\rfloor+i}(t)-\delta_{j}(t)\right)
$$

with $N_{j}:=\left\lfloor N \theta_{j}\right\rfloor-\left\lfloor N \theta_{j-1}\right\rfloor$. Then

$$
\left\{Z_{N}^{(j, m)}(\cdot)\right\}_{j=1, \ldots, K, m=1, \ldots, M} \rightsquigarrow\left\{\sum_{i=1}^{\infty} \sqrt{\lambda_{i}} \int_{T} \phi_{i}(t) \zeta_{m}(t) d t W_{i}^{j}(\cdot)\right\}_{j=1, \ldots, K, m=1, \ldots, M}
$$

in $\ell^{\infty}([0,1])^{M K}$; here $\phi_{i}$ and $\lambda_{i}$ are the eigenfunctions and eigenvalues of the integral operator corresponding to the error process $\eta_{j}:=f\left(\varepsilon_{j}, \varepsilon_{j-1}, \ldots\right)$ and $\left\{W_{i}^{j}\right\}_{i, j \in \mathbb{N}}$ is an array of independent Brownian motions. The proof is similar to the proof of Lemma B.1 and details are omitted for the sake of brevity.

We now sketch the proof of Remark 3.2. Let $\hat{\mathbb{D}}_{N, j}^{c p}(\lambda, \hat{\theta}), \hat{\mathbb{D}}_{N}^{L^{2}}(\lambda, \hat{\theta})$ and $\hat{\mathbb{V}}_{N}^{L^{2}}(\hat{\theta})$ be defined as in (3.15), 3.16) and (3.17), respectively, and let $\mathbb{D}_{N, j}^{c p}(\lambda), \mathbb{D}_{N}^{L^{2}}(\lambda)$ and $\mathbb{V}_{N}^{L^{2}}$ denote the corresponding quantities with $\hat{\theta}$ replaced by the vector of true change points. Now similar arguments as given in the proof of Theorem 3.1 show that

$$
\frac{\hat{\mathbb{D}}_{N}^{L^{2}}(1, \hat{\theta})-\sum_{j=1}^{K} \Psi_{j}}{\hat{\mathbb{V}}_{N}^{L^{2}}(\hat{\theta})}=\frac{\mathbb{D}_{N}^{L^{2}}(1)-\sum_{j=1}^{K} \Psi_{j}}{\mathbb{V}_{N}^{L^{2}}}+o_{P}(1) .
$$

Consequently, it is sufficient to establish the weak convergence of the right-hand side of (B.42) to $\mathbb{W}$. To this end consider the partial sum processes

$$
S_{N, j}(t, \lambda)=\frac{1}{N_{j}} \sum_{i=1}^{\left\lfloor\lambda N_{j}\right\rfloor} X_{\left\lfloor N \theta_{j-1}\right\rfloor+i}(t) \quad(j=1, \ldots, K+1) .
$$


Then

$$
\mathbb{D}_{N, j}^{c p}(\lambda)=\int_{T}\left\{S_{N, j+1}(t, \lambda)-S_{N, j}(t, \lambda)\right\}^{2} d t \quad(j=1, \ldots, K) .
$$

Now a similar calculation as in the proof of Theorem 3.1 shows

$$
\mathbb{D}_{N, j}^{c p}(\lambda)-\lambda^{2} \Psi_{j}=-2 \lambda \int_{T}\left\{\delta_{j}(t)-\delta_{j-1}(t)\right\}\left\{\tilde{S}_{N, j+1}(t, \lambda)-\tilde{S}_{N, j}(t, \lambda)\right\} d t+o_{\mathbb{P}}\left(N^{-1 / 2}\right)
$$

where $\tilde{S}_{N, j}$ are centered versions of $S_{N, j}$ defined in the beginning of this proof.

Applying (B.41) for the collection $\zeta_{m}=\delta_{m}-\delta_{m-1}, m=1, \ldots, K+1$ in combination with the continuous mapping theorem shows that

$$
\begin{aligned}
\left\{\sqrt{N}\left(\mathbb{D}_{N}^{c p}(\lambda)-\lambda^{2} \sum_{j=1}^{K} \Psi_{j}\right)\right\}_{\lambda \in[0,1]} & \\
& \rightsquigarrow\left\{2 \lambda \sum_{j=1}^{K} \sum_{i=1}^{\infty} \sqrt{\lambda_{i}} \int\left\{\delta_{j}(t)-\delta_{j-1}(t)\right\} \phi_{i}(t) d t\left(\frac{W_{i}^{j}(\lambda)}{\sqrt{\theta_{j+1}-\theta_{j}}}-\frac{W_{i}^{j+1}(\lambda)}{\sqrt{\theta_{j+2}-\theta_{j+1}}}\right)\right\}_{\lambda \in[0,1]}
\end{aligned}
$$

After some tedious but straightforward covariance manipulations it follows that the process on the right-hand side above has the same distribution as $\left\{\tau^{2} \lambda \mathbb{B}(\lambda)\right\}_{\lambda \in[0,1]}$ where $\mathbb{B}$ denotes a standard Brownian motion and $\tau^{2}$ is a constant depending on various quantities in model (3.11). Now exactly the same arguments as given in the second part of Section B.1.1 show the weak convergence of the right-hand side of $(\mathrm{B} .42)$ to the random variable $\mathbb{W}$ which completes the proof.

\section{B.4 Outline of proofs for Section 4}

We begin by some preliminary observations. Assume that $\left\{X_{j}\right\}_{j \in \mathbb{Z}}$ is a functional time series with values in $L^{2}([0,1])$ satisfying (A1) with mean $\mu$ and errors $\eta_{j}$ and that those errors satisfy (A2), (A3'), (A4'). Note that the functions $(X \otimes X)(s, t)=X(s) X(t)$ can be interpreted as random elements in $L^{2}\left([0,1]^{2}\right)$ since

$$
\int_{T} \int_{T}\left\{X_{1}(s) X_{1}(t)\right\}^{2} d s d t=\left\|X_{1}\right\|_{2}^{4}<\infty .
$$

Moreover, by some elementary computations

$$
\sum_{\ell=1}^{\infty}\left(\mathbb{E}\left\|\eta_{0} \otimes \eta_{0}-\mathbb{E}\left[\eta_{0} \otimes \eta_{0}\right]-\eta_{0, \ell} \otimes \eta_{0, \ell}+\mathbb{E}\left[\eta_{0, \ell} \otimes \eta_{0, \ell}\right]\right\|^{2+\psi / 2}\right)^{2 / \kappa}<\infty
$$

where $\|\cdot\|$ now denotes the $L^{2}$ norm on $L^{2}\left([0,1]^{2}\right)$, see also the proof of Lemma A.3 in Aue et al. (2018) for similar arguments. Now going through the proofs in Berkes et al. (2013) we find that most of their results hold for spaces of square integrable functions on general subsets of $\mathbb{R}^{d}$ 
and that there is no special structure of $T=[0,1]$ that they use. In particular, we obtain the following generalizations of the results in Berkes et al. (2013). Let

$$
\tilde{S}_{n}((s, t), \lambda):=\frac{1}{n} \sum_{j=1}^{\lfloor n \lambda\rfloor} \eta_{j}(t) \eta_{j}(s)
$$

and equip

$$
\mathcal{G}_{2}:=\left\{f: T^{2} \times[0,1] \rightarrow \mathbb{R}: \sup _{\lambda \in[0,1]} \int_{T} \int_{T} f^{2}((s, t), \lambda) d s d t<\infty\right\}
$$

with the norm

$$
\|f\|_{\mathcal{G}_{2}}:=\sup _{\lambda \in[0,1]} \int_{T} \int_{T} f^{2}((s, t), \lambda) d s d t .
$$

A generalization of Theorem 1.1 in Berkes et al. (2013) implies that there exists a sequence of measurable random elements in $\mathcal{G}_{2}$, say $\left\{\Gamma_{n}(s, t, \lambda)\right\}_{\lambda, s, t \in[0,1]}$, such that

$$
\begin{aligned}
& \sup _{\lambda \in[0,1]}\left\|\sqrt{n} \tilde{S}_{n}(\cdot, \lambda)-\Gamma_{n}(\cdot, \lambda)\right\|=o_{\mathbb{P}}(1) \\
& \left\{\Gamma_{n}((s, t), \lambda)\right\}_{\lambda, t \in[0,1]} \stackrel{\mathcal{D}}{=}\{\Gamma((s, t), \lambda)\}_{\lambda, s, t \in[0,1]},
\end{aligned}
$$

where $\Gamma$ is defined by

$$
\Gamma((s, t), \lambda)=\sum_{i=1}^{\infty} \sqrt{\lambda_{i}} \phi_{i}(s, t) W_{i}(\lambda) .
$$

$\left\{W_{i}\right\}_{i \in \mathbb{N}}$ is a sequence of independent Brownian motions and $\lambda_{i}, \phi_{i}$ are the eigenvalues and (orthonormal) eigenfunctions of the integral operator corresponding to the covariance kernel

$$
\begin{aligned}
C\left((s, t),\left(s^{\prime}, t^{\prime}\right)\right):= & \operatorname{Cov}\left(X_{0} \otimes X_{0}(s, t), X_{0} \otimes X_{0}\left(s^{\prime}, t^{\prime}\right)\right)+\sum_{\ell=1}^{\infty} \operatorname{Cov}\left(X_{0} \otimes X_{0}(s, t), X_{\ell} \otimes X_{\ell}\left(s^{\prime}, t^{\prime}\right)\right) \\
& +\sum_{\ell=1}^{\infty} \operatorname{Cov}\left(X_{0} \otimes X_{0}(s, t), X_{-\ell} \otimes X_{-\ell}\left(s^{\prime}, t^{\prime}\right)\right),
\end{aligned}
$$

i.e.

$$
C\left((s, t),\left(s^{\prime}, t^{\prime}\right)\right)=\sum_{i=1}^{\infty} \lambda_{i} \phi_{i}(s, t) \phi_{i}\left(s^{\prime}, t^{\prime}\right)
$$

with

$$
\lambda_{i} \phi_{i}(s, t)=\int_{T} \int_{T} C\left(\left(s^{\prime}, t^{\prime}\right),(s, t)\right) \phi_{i}\left(s^{\prime}, t^{\prime}\right) d s^{\prime} d t^{\prime} \quad(i \in \mathbb{N}) .
$$

A generalization of Lemma 2.2 in Berkes et al. (2013) further shows that $\sum_{k} \lambda_{k}<\infty$ and that

$$
\sup _{0 \leq \lambda \leq 1} \int_{T} \Gamma^{2}((s, t), \lambda) d t<\infty \quad \text { a.s. }
$$


The latter implies that for any square integrable function $\zeta:[0,1]^{2} \rightarrow \mathbb{R}$ the process

$$
\left\{\int_{T} \int_{T} \zeta(s, t) \Gamma((s, t), \lambda) d s d t\right\}_{\lambda \in[0,1]}
$$

can be viewed as an element of $\ell^{\infty}([0,1])$; the same is true for the process $\left\{\int_{T} \Gamma^{2}((s, t), \lambda) d t\right\}_{\lambda \in[0,1]}$. Moreover, summability of the sequence $\left(\lambda_{k}\right)_{k \in \mathbb{N}}$ together with properties of the modulus of continuity of Brownian motions and similar arguments as give in the derivation of (B.8) imply that for any positive sequence $\left(\kappa_{k}\right)_{k \in \mathbb{N}}$ such that $\kappa_{n} \rightarrow 0$

$$
\sup _{\substack{\nu, \lambda \in[0,1]: \\|\nu-\lambda| \leq \kappa_{n}}} \int_{T}\left\{\Gamma^{2}((s, t), \lambda)-\Gamma^{2}((s, t), \nu)\right\}^{2} d t=o_{\mathbb{P}}(1) \quad\left(\kappa_{n} \rightarrow 0\right) .
$$

\section{B.4.1 Outline of proof for Section 4.1}

Observe that under the assumptions made we have

$$
\sup _{\lambda \in[0,1]}\left\|\frac{1}{m} \sum_{i=1}^{\lfloor m \lambda\rfloor} \eta_{i}^{X}\right\|_{2}^{2}=O_{P}\left(m^{-1}\right)
$$

and the same is true with $\eta_{i}^{Y}$ and $n$ replacing $m$. Next note that we have for any $\lambda \geq \varepsilon>0$ for all $m>1 / \varepsilon$

$$
\begin{aligned}
& \frac{1}{m-1} \sum_{j=1}^{\lfloor m \lambda\rfloor}\left\{\left(X_{j}(s)-\frac{1}{\lfloor m \lambda\rfloor \vee 1} \sum_{i=1}^{\lfloor m \lambda\rfloor} X_{i}(s)\right)\left(X_{j}(t)-\frac{1}{\lfloor m \lambda\rfloor \vee 1} \sum_{i=1}^{\lfloor m \lambda\rfloor} X_{i}(t)\right)-\eta_{j}^{X}(t) \eta_{j}^{X}(s)\right\} \\
= & \frac{1}{m-1} \sum_{j=1}^{\lfloor m \lambda\rfloor}\left\{\left(\eta_{j}^{X}(s)-\frac{1}{\lfloor m \lambda\rfloor \vee 1} \sum_{i=1}^{\lfloor m \lambda\rfloor} \eta_{i}^{X}(s)\right)\left(\eta_{j}^{X}(t)-\frac{1}{\lfloor m \lambda\rfloor \vee 1} \sum_{i=1}^{\lfloor m \lambda\rfloor} \eta_{i}^{X}(t)\right)-\eta_{j}^{X}(t) \eta_{j}^{X}(s)\right\} \\
= & -\frac{\lfloor m \lambda\rfloor \vee 1}{m-1}\left(\frac{1}{\lfloor m \lambda\rfloor \vee 1} \sum_{i=1}^{\lfloor m \lambda\rfloor} \eta_{i}^{X}(s)\right)\left(\frac{1}{\lfloor m \lambda\rfloor \vee 1} \sum_{i=1}^{\lfloor m \lambda\rfloor} \eta_{i}^{X}(t)\right) .
\end{aligned}
$$

Define

$$
\widetilde{D}_{n, m}(s, t, \lambda):=\frac{1}{m} \sum_{j=1}^{\lfloor m \lambda\rfloor} \eta_{j}^{X}(t) \eta_{j}^{X}(s)-\frac{1}{n} \sum_{j=1}^{\lfloor n \lambda\rfloor} \eta_{j}^{Y}(t) \eta_{j}^{Y}(s) .
$$

The above calculation combined with (B.51) shows that

$$
\sup _{1 \geq \lambda \geq \varepsilon} \int_{T} \int_{T}\left\{D_{n, m}(s, t, \lambda)-\tilde{D}_{n, m}(s, t, \lambda)\right\}^{2} d s d t=o_{P}\left((n+m)^{-1}\right) .
$$

Indeed, observe that

$$
\begin{aligned}
D_{n, m}(s, t, \lambda)-\tilde{D}_{n, m}(s, t, \lambda)= & -\frac{\lfloor m \lambda\rfloor \vee 1}{m-1}\left(\frac{1}{\lfloor m \lambda\rfloor \vee 1} \sum_{i=1}^{\lfloor m \lambda\rfloor} \eta_{i}^{X}(s)\right)\left(\frac{1}{\lfloor m \lambda\rfloor \vee 1} \sum_{i=1}^{\lfloor m \lambda\rfloor} \eta_{i}^{X}(t)\right) \\
& +\frac{\lfloor n \lambda\rfloor \vee 1}{n-1}\left(\frac{1}{\lfloor n \lambda\rfloor \vee 1} \sum_{i=1}^{\lfloor n\rfloor} \eta_{i}^{Y}(s)\right)\left(\frac{1}{\lfloor n \lambda\rfloor \vee 1} \sum_{i=1}^{\lfloor n \lambda\rfloor} \eta_{i}^{Y}(t)\right)
\end{aligned}
$$


It suffices to bound both terms on the right individually. Since $\lambda \geq \varepsilon$ we have $m /\lfloor m \lambda\rfloor=O(1)$. Moreover

$$
\begin{aligned}
& \int_{t} \int_{T}\left\{\left(\frac{1}{\lfloor m \lambda\rfloor \vee 1} \sum_{i=1}^{\lfloor m \lambda\rfloor} \eta_{i}^{X}(s)\right)\left(\frac{1}{\lfloor m \lambda\rfloor \vee 1} \sum_{i=1}^{\lfloor m \lambda\rfloor} \eta_{i}^{X}(t)\right)\right\}^{2} d s d t \\
= & \left\|\frac{1}{\lfloor m \lambda\rfloor \vee 1} \sum_{i=1}^{\lfloor m \lambda\rfloor} \eta_{i}^{X}\right\|^{2}\left\|\frac{1}{\lfloor m \lambda\rfloor \vee 1} \sum_{i=1}^{\lfloor m \lambda\rfloor} \eta_{i}^{X}\right\|^{2} \\
= & O(1)\left\|\frac{1}{m} \sum_{i=1}^{\lfloor m \lambda\rfloor} \eta_{i}^{X}\right\|^{4}=O_{P}\left(m^{-2}\right)
\end{aligned}
$$

where the last inequality follows from B.51. The other term can be bounded similarly. Hence by a simple calculation involving the Cauchy-Schwarz inequality we find that

$$
\begin{aligned}
\sqrt{n+m} \hat{\mathbb{D}}_{n, m}^{C} & =\int_{T} \int_{T} \widetilde{D}_{n, m}(s, t, \lambda)^{2} d s d t+o_{P}(1) \\
\sqrt{n+m} \hat{\mathbb{V}}_{m, n}^{C} & =\left(\int_{0}^{1}\left[\int_{T} \int_{T} \widetilde{D}_{m, n}(s, t, \lambda)^{2} d s d t-\lambda^{2} \int_{T} \int_{T} \widetilde{D}_{m, n}(s, t, 1)^{2} d s d t\right]^{2} \nu(d \lambda)\right)^{1 / 2}+o_{P}(1)
\end{aligned}
$$

where we used the fact that $\nu$ does not place any mass in a neighbourhood of zero in the second identity. Hence it suffices to analyze the test based on $\widetilde{D}_{m, n}$ instead of $D_{m, n}$. This can now be done by exactly the same arguments as in the proof of Theorem 2.2 after replacing (B.44)-(B.46) in that proof by $(\mathrm{B} .2)-(\mathrm{B} .4)$. Details are omitted for the sake of brevity.

\section{B.4.2 Outline of proof for Section 4.2}

Similarly as in the two sample case, the arguments are very similar to those given in Section B.2 and hence we only outline the main steps. We begin by stating an extension of Lemma B.1 in Section B.2.1. Recall the model described in 4.3) and define $\eta_{i}:=X_{i}-\mu$. By assumption, $\eta_{i}^{(k)}$ satisfy (A4').

Lemma B.2 In the setting above consider a fixed (but arbitrary) function $\zeta$ in $L^{2}\left(T^{2}\right)$. For $\lambda \in[0,1]$ define the processes

$$
\tilde{Z}_{N}^{(k)}(\lambda):=\frac{1}{\sqrt{N}} \sum_{i=1}^{\lfloor N \lambda\rfloor} \int_{T} \int_{T} \eta_{i}^{(k)}(s) \eta_{i}^{(k)}(t) \zeta(s, t) d s d t, \quad k=1,2,
$$

then

$$
\left(\tilde{Z}_{N}^{(1)}, \tilde{Z}_{N}^{(2)}\right)^{\top} \rightsquigarrow \Sigma^{1 / 2}\left(\mathbb{B}_{1}, \mathbb{B}_{2}\right)^{\top} \text { in } \ell^{\infty}([0,1])^{2},
$$

where $\mathbb{B}_{1}, \mathbb{B}_{2}$ are two independent standard Brownian motions on the interval $[0,1]$ and $\Sigma$ is a symmetric $2 \times 2$ matrix with finite entries given by

$$
\Sigma_{i j}=\sum_{h \in \mathbb{Z}} \int_{T} \int_{T} \int_{T} \int_{T} \operatorname{Cov}\left(\eta_{0}^{(i)} \otimes \eta_{0}^{(i)}(s, t), \eta_{h}^{(j)} \otimes \eta_{h}^{(j)}\left(s^{\prime}, t^{\prime}\right)\right) \zeta(s, t) \zeta\left(s^{\prime}, t^{\prime}\right) d s d t d s^{\prime} d t^{\prime} .
$$


The proof of this Lemma follows by very similar arguments as the proof of Lemma B.1 upon observing that all results from Berkes et al. (2013) used in that proof continue to hold with $\eta_{i}$ replaced by $\eta_{i} \otimes \eta_{i}, L^{2}(T)$ replaced by $L^{2}\left(T^{2}\right)$ upon noting that under (A4') the $\eta_{i} \otimes \eta_{i}$ satisfy (B.43).

The next key step is to prove that $\hat{\theta}^{C o v}=\theta_{0}+o_{P}\left(N^{-1 / 2}\right)$. Begin by observing that

$$
\begin{aligned}
\hat{C}_{1: k}(s, t) & =\frac{1}{k-1} \sum_{i=1}^{k} \eta_{i}(s) \eta_{i}(t)-\frac{k}{k-1}\left(\frac{1}{k} \sum_{i=1}^{k} \eta_{i}(s)\right)\left(\frac{1}{k} \sum_{i=1}^{k} \eta_{i}(t)\right), \\
\hat{C}_{k+1: N}(s, t) & =\frac{1}{N-k-1} \sum_{i=k+1}^{N} \eta_{i}(s) \eta_{i}(t)-\frac{N-k}{N-k-1}\left(\frac{1}{N-k} \sum_{i=k+1}^{N} \eta_{i}(s)\right)\left(\frac{1}{N-k} \sum_{i=k+1}^{N} \eta_{i}(t)\right) .
\end{aligned}
$$

Noting that (B.51) holds with $N, \eta_{i}^{(j)}(j=1,2)$ instead of $m, \eta_{i}^{X}$ shows after some computations that

$$
\begin{gathered}
\sup _{\lfloor N \varepsilon\rfloor+1 \leq k \leq N-\lfloor N \varepsilon\rfloor} \int_{T} \int_{T}\left\{\frac{k}{k-1}\left(\frac{1}{k} \sum_{i=1}^{k} \eta_{i}(s)\right)\left(\frac{1}{k} \sum_{i=1}^{k} \eta_{i}(t)\right)\right\}^{2} d s d t=O_{P}\left(N^{-2}\right) \\
\sup _{\lfloor N \varepsilon\rfloor+1 \leq k \leq N-\lfloor N \varepsilon\rfloor} \int_{T} \int_{T}\left\{\frac{N-k}{N-k-1}\left(\frac{1}{N-k} \sum_{i=k+1}^{N} \eta_{i}(s)\right)\left(\frac{1}{N-k} \sum_{i=k+1}^{N} \eta_{i}(t)\right)\right\}^{2} d s d t=O_{P}\left(N^{-2}\right) .
\end{gathered}
$$

Define

$$
\tilde{C}_{1: k}:=\frac{1}{k-1} \sum_{i=1}^{k} \eta_{i} \otimes \eta_{i}, \quad \tilde{C}_{k+1: k}:=\frac{1}{N-k-1} \sum_{i=k+1}^{N} \eta_{i} \otimes \eta_{i} .
$$

Next observe that

$$
\sup _{\lfloor N \varepsilon\rfloor+1 \leq k \leq N-\lfloor N \varepsilon\rfloor}\left\|\frac{1}{k} \sum_{i=1}^{k} \eta_{i} \otimes \eta_{i}-\mathbb{E}\left[\eta_{i} \otimes \eta_{i}\right]\right\|_{L^{2}\left(T^{2}\right)}+\left\|\frac{1}{N-k} \sum_{i=k+1}^{N} \eta_{i} \otimes \eta_{i}-\mathbb{E}\left[\eta_{i} \otimes \eta_{i}\right]\right\|_{L^{2}\left(T^{2}\right)}=O_{P}\left(N^{-1 / 2}\right) .
$$

This in particular implies that

$$
\sup _{\lfloor N \varepsilon\rfloor+1 \leq k \leq N-\lfloor N \varepsilon\rfloor}\left\|\tilde{C}_{1: k}\right\|+\left\|\tilde{C}_{k+1: N}\right\|=O_{P}(1) .
$$

Hence by the reverse triangle inequality and the usual triangle inequality we have

$$
\begin{aligned}
& \sup _{\lfloor N \varepsilon\rfloor+1 \leq k \leq N-\lfloor N \varepsilon\rfloor}\left|\hat{f}^{C o v}(k)-\left\|\tilde{C}_{1: k}-\tilde{C}_{k+1: N}\right\|_{L^{2}\left(T^{2}\right)}^{2}\right| \\
= & \sup _{\lfloor N \varepsilon\rfloor+1 \leq k \leq N-\lfloor N \varepsilon\rfloor}\left|\left\|\hat{C}_{1: k}-\hat{C}_{k+1: N}\right\|_{L^{2}\left(T^{2}\right)}^{2}-\left\|\tilde{C}_{1: k}-\tilde{C}_{k+1: N}\right\|_{L^{2}\left(T^{2}\right)}^{2}\right| \\
\leq & \sup _{\lfloor N \varepsilon\rfloor+1 \leq k \leq N-\lfloor N \varepsilon\rfloor}\left\{\left\|\tilde{C}_{1: k}-\hat{C}_{1: k}\right\|+\left\|\tilde{C}_{k+1: N}-\hat{C}_{k+1: N}\right\|\right\}\left\{\left\|\tilde{C}_{1: k}\right\|+\left\|\hat{C}_{1: k}\right\|+\left\|\tilde{C}_{k+1: N}\right\|+\left\|\hat{C}_{k+1: N}\right\|\right\} \\
= & o_{P}\left(N^{-1 / 2}\right) .
\end{aligned}
$$


Hence, applying the same arguments as in the proof of Proposition 3.1 but replacing all instances $X_{i}, \mathbb{E}\left[X_{i}\right]$ there by $\eta_{i} \otimes \eta_{i}, \mathbb{E}\left[\eta_{i} \otimes \eta_{i}\right]$ and making corresponding adjustments to integrals and norms we find that $\hat{\theta}^{\text {Cov }}=\theta_{0}+o_{P}\left(N^{-1 / 2}\right)$.

The remaining proof consists in observing that by similar arguments as above we have uniformly in $\theta \in[\varepsilon, 1-\varepsilon], \lambda \in[\varepsilon, 1]$

$$
\begin{aligned}
\| D_{N}^{c p, C o v}(\cdot, \lambda, \theta)-\frac{1}{\lfloor N \theta\rfloor-1} \sum_{j=1}^{\lfloor N \theta\rfloor \lambda\rfloor} \eta_{j} \otimes \eta_{j}- & \\
& \frac{1}{N-\lfloor N \theta\rfloor-1} \sum_{j=\lfloor\theta N\rfloor+1}^{\lfloor\theta N\rfloor+\lfloor\lambda(N-\lfloor\theta N\rfloor)\rfloor} \eta_{j} \otimes \eta_{j} \|_{L^{2}\left(T^{2}\right)}=o_{P}\left(N^{-1 / 2}\right)
\end{aligned}
$$

and following similar arguments as in the proof of Theorem 3.1 .

\section{Extensions beyond functional time series}

In this section we briefly discuss how the ideas presented in this paper can be extended beyond the context of functional time series. We begin by introducing a general setup which will be used throughout this section. Let $X_{1}, \ldots, X_{n}$ denote a sample of (potentially dependent) random elements in some measurable space $\mathcal{S}$. Assume that we are interested in inference on a parameter

$\mu=\mu_{\mathbb{P}}$ that can be assigned to distributions $\mathbb{P}$ on $\mathcal{S}$. We will assume that $\mu$ takes values in $\mathcal{M}$, a subset of a real Hilbert space $\mathcal{H}$ equipped with an inner product $\langle\cdot, \cdot\rangle$ and induced norm by $\|\cdot\|_{\mathcal{H}}$. Further, assume that for each $m \in \mathbb{N}$ there exists a mapping $f_{m}: \mathcal{S}^{m} \rightarrow \mathcal{M}$, where $f_{m}\left(X_{1}, \ldots, X_{m}\right)$ is interpreted as estimator for $\mu$ based on the observations $X_{1}, \ldots, X_{m}$. The situation considered in Sections 1 - 3 corresponds to the choice $\mathcal{S}=L^{2}([0,1]), \mathcal{H}=L^{2}([0,1])$ and $\mu_{\mathbb{P}}$ is the mean (function) of $X_{i}$ (see the discussion in Example C.1 below for more details). Finally, define for $\Lambda \subset[0,1]$ the space of functions

$$
\mathcal{B}(\Lambda, \mathcal{H}):=\left\{f: \Lambda \rightarrow \mathcal{H}: \sup _{\lambda \in \Lambda}\|f(\lambda)\|_{\mathcal{H}}<\infty\right\}
$$

equipped with the norm

$$
\|g\|_{\mathcal{B}}:=\sup _{\lambda \in \Lambda}\|g(\lambda)\|_{\mathcal{H}}
$$

This space will be used to characterize the joint behaviour of estimators of $\mu$ computed from several sub-samples (with sub-sample proportion corresponding to the index $\lambda$ ). Note that if the set $\Lambda$ contains only finitely many elements, say $|\Lambda|$, the normed space $\left(\mathcal{B}(\Lambda, \mathcal{H}),\|\cdot\|_{\mathcal{B}}\right)$ can be identified with the $|\Lambda|$-fold Cartesian product of $\mathcal{H}$ (viewed as a normed space).

\section{C.1 The one sample and two sample case}

We begin by considering the one sample case since in this setting the relevant conditions are particularly simple and transparent. The self-normalized statistic is based on a probability 
measure $\nu$ with support $\Lambda_{\nu} \subset(0,1)$. Using the notation $\hat{\mu}_{1: k}:=f_{k}\left(X_{1}, \ldots, X_{k}\right)$ we define random elements in $\mathcal{B}(\Lambda, \mathcal{H})$ through $g_{n}(\lambda):=\lambda \sqrt{n}\left(\hat{\mu}_{1:\lfloor\lambda n\rfloor}-\mu\right)$. Assume that

$$
g_{n} \rightsquigarrow \mathbb{H} \quad \text { in } \mathcal{B}\left(\Lambda_{\nu} \cup 1, \mathcal{H}\right)
$$

Note that we do not require measurability of $g_{n}$ and weak convergence is defined in the sense of Hoffman-Jorgensen, see Section 1.3 in Van der Vaart and Wellner (1996). Further, assume that the limit $\mathbb{H}$ has the following additional properties:

$$
\begin{aligned}
& \{\langle\mathbb{H}(\lambda), \mu\rangle\}_{\lambda \in \Lambda_{\nu} \cup 1} \stackrel{\mathcal{D}}{=} \sigma^{2}\{\lambda \mathbb{B}(\lambda)\}_{\lambda \in \Lambda_{\nu} \cup 1}, \\
& \left(\int_{\Lambda_{\nu}}\left[\|\mathbb{H}(\lambda)\|_{\mathcal{H}}^{2}-\lambda^{2}\|\mathbb{H}(1)\|_{\mathcal{H}}^{2}\right]^{2} \nu(d \lambda)\right)^{-1}=O_{P}(1),
\end{aligned}
$$

where $\sigma^{2}$ is a (nonnegative) real-valued parameter that can depend on the distribution $\mathbb{P}$ of $X_{i}$ and $\mathbb{B}$ is a standard Brownian motion on the interval $[0,1]$. Moreover, we assume that $\sigma^{2} \neq 0$ if $\mu \neq 0$.

If (C.1) - C.3 are satisfied, an asymptotic level $\alpha$ and consistent test for the (relevant) hypotheses

$$
H_{0}:\|\mu\|_{\mathcal{H}}^{2} \leq \Delta \quad \text { versus } \quad H_{1}:\|\mu\|_{\mathcal{H}}^{2}>\Delta
$$

is given by rejecting $H_{0}$ whenever the inequality 2.8 holds, where the statistic $\hat{\mathbb{T}}_{n}$ and $\hat{\mathbb{V}}_{n}$ are now defined by $\hat{\mathbb{T}}_{n}:=\left\|\hat{\mu}_{1: n}\right\|_{\mathcal{H}}^{2}$ and

$$
\hat{\mathbb{V}}_{n}:=\left(\int_{\Lambda_{\nu}}\left[\lambda^{2}\left\|\hat{\mu}_{1:\lfloor n \lambda]}\right\|_{\mathcal{H}}^{2}-\lambda^{4}\left\|\hat{\mu}_{1: n}\right\|_{\mathcal{H}}^{2}\right]^{2} \nu(d \lambda)\right)^{1 / 2}
$$

respectively, and $q_{1-\alpha}(\mathbb{W})$ is the $(1-\alpha)$-quantile of the distribution of the random variable $\mathbb{W}$ defined in 2.9 .

\section{Example C.1}

(a) In the setting of Section 2.1 the random variables $X_{1}, \ldots, X_{n}$ with distribution $\mathbb{P}$ take values in $\mathcal{S}=L^{2}([0,1]), \mu_{\mathbb{P}}$ is the mean (function) of $X_{i}$ which is an element of $\mathcal{H}=L^{2}([0,1])$, and $f_{m}\left(X_{1}, \ldots, X_{m}\right)=m^{-1}\left(X_{1}+\ldots+X_{m}\right)$. The estimators $\hat{\mu}_{1:\lfloor n \lambda\rfloor}$ then take the form $\hat{\mu}_{1:\lfloor n \lambda\rfloor}(t)=\frac{1}{\lfloor n \lambda\rfloor} \sum_{i=1}^{\lfloor n \lambda\rfloor} X_{i}(t)$. The limit $\mathbb{H}$ in C.1] is given by $\mathbb{H}(\lambda)=\Gamma(\cdot, \lambda)$ where the process $\Gamma$ is defined in equation (B.4.

(b) When $\mathcal{H}$ is $\mathbb{R}$ with $\langle x, y\rangle=x y$, the space $\mathcal{B}(\Lambda, \mathcal{H})$ can be identified with $\ell^{\infty}(\Lambda)$. Conditions (C.1) and (C.2) follow from the functional CLT

$$
\left\{\lambda \sqrt{n}\left(\hat{\mu}_{1:\lfloor\lambda n\rfloor}-\mu\right)\right\}_{\lambda \in \Lambda_{\nu} \cup 1} \rightsquigarrow \tau^{2}\{\mathbb{B}(\lambda)\}_{\lambda \in \Lambda_{\nu} \cup 1} .
$$

In this case $\mathbb{H}=\tau^{2} \mathbb{B}$ and $\sigma^{2}=\mu^{2} \tau^{2}$. Moreover, condition (C.3) follows from elementary properties of the multivariate normal distribution and the assumption that $\nu$ is a probability 
measure on the interval $(0,1)$.

When $\mu$ is the mean of $\mathbb{P}$ and $\hat{\mu}_{1:\lfloor\lambda n\rfloor}$ is the sample mean of the first $\lfloor\lambda n\rfloor$ observations, (C.4) holds under a wide variety of assumptions on the serial dependence of time series. When $\mathcal{S}=\mathbb{R}^{d}$ and $\mu$ is a smooth function of the (multivariate) cdf of $X$, this condition can be verified using the general framework developed in Volgushev and Shao (2014). This includes quantities such as quantiles or Kendall's $\tau$ and other dependence measures.

(c) If $\mathcal{H}=\mathbb{R}^{d}$ (with the usual inner product), the space $\mathcal{B}(\Lambda, \mathcal{H}$ ) can be identified with $\left[\ell^{\infty}(\Lambda)\right]^{d}$. The norm $\|f\|_{\mathcal{B}}=\sup _{\lambda \in \Lambda}\|f(\lambda)\|_{2}\left(f \in \mathcal{B}\left(\Lambda, \mathbb{R}^{d}\right)\right)$ is equivalent to the usual norm $\left\|\left(g_{1}, \ldots, g_{d}\right)\right\|:=\max _{j=1, \ldots, d} \sup _{\lambda \in \Lambda}\left|g_{j}(\lambda)\right|$ where $\left(g_{1}, \ldots, g_{d}\right) \in\left[\ell^{\infty}(\Lambda)\right]^{d}$ and the weak convergence in C.1 is interpreted as weak convergence in the product space $\left[\ell^{\infty}(\Lambda)\right]^{d}$ as discussed in Section 1.4 of Van der Vaart and Wellner (1996). Let, $\hat{\mu}_{1:\lfloor\lambda n, j}$ and $\mu_{j}$ denote the $j$ 'th components of the vectors $\hat{\mu}_{1:\lfloor\lambda n\rfloor}$ and $\mu$, respectively, and let $g_{n, j}(\lambda):=\lambda \sqrt{n}\left(\hat{\mu}_{1:\lfloor\lambda n\rfloor, j}-\mu_{j}\right)$. Conditions (C.1) and C.2 now follow from a multivariate version of the functional CLT, i.e.

$$
\left(g_{n, 1}, \ldots, g_{n, d}\right)^{\top} \rightsquigarrow \Sigma\left(\mathbb{B}_{1}, \ldots, \mathbb{B}_{d}\right)^{\top}
$$

in $\left[\ell^{\infty}(\Lambda)\right]^{d}$, where $\mathbb{B}_{1}, \ldots, \mathbb{B}_{d}$ are independent Brownian motions on the interval $[0,1]$ and $\Sigma$ denotes a matrix which can depend on $\mathbb{P}$ and is non-singular when $\mu \neq 0$. The functional weak convergence in C.5 can be verified in a similar fashion as discussed in (b) and details are omitted for the sake of brevity.

Next we briefly discuss the two sample case, where $X_{1}, \ldots, X_{m}$ and $Y_{1}, \ldots, Y_{n}$ are random variables with $X_{i} \sim \mathbb{P}, Y_{i} \sim \mathbb{Q}$. For simplicity we shall further assume that the samples $X_{1}, \ldots, X_{m}$ and $Y_{1}, \ldots, Y_{n}$ are independent, while dependence within the samples is explicitly allowed. Let $\mu_{\mathbb{P}}, \mu_{\mathbb{Q}}$ denote the parameters of interest corresponding to $\mathbb{P}, \mathbb{Q}$, respectively. Introduce the notation $\hat{\mu}_{1: k}^{X}:=f_{k}\left(X_{1}, \ldots, X_{k}\right), \hat{\mu}_{1: k}^{Y}:=f_{k}\left(Y_{1}, \ldots, Y_{k}\right)$ and let $g_{m}^{X}(\lambda):=\lambda \sqrt{m}\left(\hat{\mu}_{1:\lfloor\lambda m\rfloor}^{X}-\mu_{\mathbb{P}}\right), g_{n}^{Y}(\lambda):=$ $\lambda \sqrt{n}\left(\hat{\mu}_{1:\lfloor\lambda n\rfloor}^{Y}-\mu_{\mathbb{Q}}\right)$. Provided that $m /(m+n) \rightarrow \rho \in(0,1)$ and $g_{m}^{X} \rightsquigarrow \mathbb{H}^{X}$ and $g_{n}^{Y} \rightsquigarrow \mathbb{H}^{Y}$ in $\mathcal{B}\left(\Lambda_{\nu} \cup 1, \mathcal{H}\right)$ with limiting processes $\mathbb{H}^{X}, \mathbb{H}^{Y}$ satisfying (C.2) and C.3), an asymptotic level $\alpha$ and consistent test for the (relevant) hypotheses

$$
H_{0}:\left\|\mu_{\mathbb{P}}-\mu_{\mathbb{Q}}\right\|_{\mathcal{H}}^{2} \leq \Delta \quad \text { versus } \quad H_{1}:\left\|\mu_{\mathbb{P}}-\mu_{\mathbb{Q}}\right\|_{\mathcal{H}}^{2}>\Delta
$$

is given by rejecting $H_{0}$ whenever $\hat{\mathbb{T}}_{m, n}>\Delta+q_{1-\alpha}(\mathbb{W}) \hat{\mathbb{V}}_{m, n}$. Here the statistics $\hat{\mathbb{T}}_{m, n}$ and $\hat{\mathbb{V}}_{m, n}$ are defined by $\hat{\mathbb{T}}_{m, n}:=\left\|\hat{\mu}_{1: m}^{X}-\hat{\mu}_{1: n}^{Y}\right\|_{\mathcal{H}}^{2}$ and

$$
\hat{\mathbb{V}}_{m, n}=\left(\int_{\Lambda_{\nu}}\left\{\lambda^{2}\left\|\hat{\mu}_{1:\lfloor m \lambda\rfloor}^{X}-\hat{\mu}_{1:\lfloor n \lambda\rfloor}^{Y}\right\|_{\mathcal{H}}^{2}-\lambda^{4}\left\|\hat{\mu}_{1: m}^{X}-\hat{\mu}_{1: n}^{Y}\right\|_{\mathcal{H}}^{2}\right\}^{2} \nu(d \lambda)\right)^{1 / 2},
$$

respectively, and $q_{1-\alpha}(\mathbb{W})$ is the $(1-\alpha)$-quantile of the distribution of the random variable $\mathbb{W}$ in 2.9. The discussion in Example C.1 also applies to the two sample case with obvious modifications. 


\section{C.2 Testing for relevant change points in a general setting}

Next we discuss the problem of testing for relevant change points in the general context introduced in the beginning of Section $\mathbb{C}$. Assume that $X_{i} \sim \mathbb{P}$ for $1 \leq i \leq n \theta_{0}$ and $X_{i} \sim \mathbb{Q}$ for $n \theta_{0}<i \leq n$ where $\theta_{0} \in(0,1)$. Technically this is a triangular array model, but we will not stress this in the notation. We explicitly allow $\mathbb{P}=\mathbb{Q}$ which corresponds to the case of no change point in the sequence $X_{1}, \ldots, X_{n}$. Let $\mu_{\mathbb{P}}, \mu_{\mathbb{Q}}$ denote the parameters of interest corresponding to $\mathbb{P}, \mathbb{Q}$, respectively. Our aim is to test the hypotheses of a relevant change in the parameter $\mu$ of the sequence $X_{1}, \ldots, X_{n}$, that is

$$
H_{0}:\left\|\mu_{\mathbb{P}}-\mu_{\mathbb{Q}}\right\|_{\mathcal{H}}^{2} \leq \Delta \quad \text { versus } \quad H_{1}:\left\|\mu_{\mathbb{P}}-\mu_{\mathbb{Q}}\right\|_{\mathcal{H}}^{2}>\Delta .
$$

Following the developments in Section 3 , we assume that $\hat{\theta}$ is a consistent estimator for $\theta_{0}$ and introduce the notation $\hat{\mu}_{j: k}:=f_{k-j+1}\left(X_{j}, \ldots, X_{k}\right)$. The null hypothesis of no relevant difference in $($ C.6 $)$ is rejected if $\hat{\mathbb{T}}^{c p}>\Delta+q_{1-\alpha}(\mathbb{W}) \hat{\mathbb{V}}^{c p}$, where the statstics $\hat{\mathbb{T}}^{c p}$ and $\hat{\mathbb{V}}^{c p}$ are defined by

$$
\begin{aligned}
\hat{\mathbb{T}}^{c p} & =\left\|\hat{\mu}_{1:\lfloor n \hat{\theta}\rfloor}-\hat{\mu}_{\lfloor n \hat{\theta}\rfloor+1: n}\right\|_{\mathcal{H}}^{2}, \\
\hat{\mathbb{V}}^{c p} & =\left(\int_{\Lambda_{\nu}}\left\{\lambda^{2}\left\|\hat{\mu}_{1:\lfloor n \lambda \hat{\theta}\rfloor}-\hat{\mu}_{\lfloor n \hat{\theta}\rfloor+1:\lfloor n \hat{\theta}\rfloor+\lfloor n \lambda(1-\hat{\theta})\rfloor}\right\|_{\mathcal{H}}^{2}-\lambda^{4}\left\|\hat{\mu}_{1:\lfloor n \hat{\theta}\rfloor}-\hat{\mu}_{\lfloor n \hat{\theta}\rfloor+1: n}\right\|_{\mathcal{H}}\right\}^{2} \nu(d \lambda)\right)^{1 / 2},
\end{aligned}
$$

respectively, and $q_{1-\alpha}(\mathbb{W})$ is the $(1-\alpha)$-quantile of the distribution of the random variable $\mathbb{W}$ in (2.9). The asymptotic validity of this test can be verified provided that

$$
\left\{\lambda \sqrt{n}\left(\hat{\mu}_{1:\lfloor\lambda \hat{\theta} n\rfloor}-\hat{\mu}_{1+\lfloor\hat{\theta} n\rfloor+\lfloor\lambda(1-\hat{\theta}) n\rfloor: n}+\mu_{\mathbb{Q}}-\mu_{\mathbb{P}}\right)\right\}_{\lambda \in \Lambda_{\nu} \cup 1} \rightsquigarrow \mathbb{H} \quad \text { in } \mathcal{B}\left(\Lambda_{\nu} \cup 1, \mathcal{H}\right),
$$

where the limiting process $\mathbb{H}$ satisfies $(\mathrm{C} .2)$ and $(\mathrm{C} .3)$. This is a non-trivial high-level assumption since the estimator $\hat{\theta}$ appears on the left-hand side.

In concrete situations, for example in the situation considered in Section 3, it is possible to replace (C.7) by more tractable and elementary assumptions. As a further illustration we will discuss the setting of a real-valued parameter $\mu$ that can be represented as smooth functional of the distribution function $F$ of the random variable $X$ with values in $\mathcal{S}=\mathbb{R}^{d}$. First, we note that there exists a general and well-developed machinery for establishing convergence rates of change point estimators [see, for example Carlstein (1988), Dümbgen (1991) or Hariz et al. (2007), among others], and we assume

$$
\hat{\theta}=\theta_{0}+o_{\mathbb{P}}\left(n^{-1 / 2}\right),
$$

throughout this section, which is satisfied in many cases of practical interest. The following discussion is a little informal and we refer the interested reader to the work of Volgushev and Shao (2014) for some of the technical details that are omitted here for the sake of brevity. Assume that $\mu_{\mathbb{P}}=\Phi\left(F_{\mathbb{P}}\right), \mu_{\mathbb{Q}}=\Phi\left(F_{\mathbb{Q}}\right)$ for some smooth map $\Phi$, where $F_{\mathbb{P}}, F_{\mathbb{Q}}$ denote the cdf of $\mathbb{P}, \mathbb{Q}$, respectively. Let $\hat{\mu}_{i: j}=\Phi\left(\hat{F}_{i: j}\right)$ where $\hat{F}_{i: j}$ is the empirical CDF of $X_{i}, \ldots, X_{j}$. Under suitable assumptions on the temporal dependence structure of $X_{1}, \ldots, X_{n}$ it is possible to prove the weak convergence 


$$
\begin{aligned}
\left(\left\{\lambda \sqrt{n}\left(\hat{F}_{1:\left\lfloor n \theta_{0} \lambda\right\rfloor}(u)-F_{\mathbb{P}}(u)\right)\right\}_{\lambda \in[0,1], u \in \mathbb{R}^{d}},\left\{\lambda \sqrt{n}\left(\hat{F}_{\left\lfloor n \theta_{0}\right\rfloor+1:\left\lfloor n \theta_{0}\right\rfloor+\left\lfloor n \lambda\left(1-\theta_{0}\right)\right\rfloor}(u)-F_{\mathbb{Q}}(u)\right)\right\}_{\lambda \in[0,1], u \in \mathbb{R}^{d}}\right) \\
\rightsquigarrow\left(\left\{\mathbb{G}_{1}(\lambda, u)\right\}_{\lambda \in[0,1], u \in \mathbb{R}^{d}},\left\{\mathbb{G}_{2}(\lambda, u)\right\}_{\lambda \in[0,1], u \in \mathbb{R}^{d}}\right)
\end{aligned}
$$

in $\ell^{\infty}\left([0,1] \times \mathbb{R}^{d}\right)^{2}$, where $\mathbb{G}_{1}, \mathbb{G}_{2}$ denote two independent centered Gaussian processes with covariance structure of the form $\mathbb{E}\left[\mathbb{G}_{j}(s, u) \mathbb{G}_{j}(t, v)\right]=(s \wedge t) K_{j}(u, v)(j=1,2)$. By elementary calculations we have

$$
\sup _{\lambda \geq \varepsilon} \sup _{u}\left|\hat{F}_{1:\left\lfloor n \theta_{0} \lambda\right\rfloor}(u)-\hat{F}_{1:\lfloor n \hat{\theta} \lambda\rfloor}(u)\right|=O_{\mathbb{P}}\left(\left|\theta_{0}-\hat{\theta}\right|\right)=o_{\mathbb{P}}\left(n^{-1 / 2}\right)
$$

(note that two empirical cdf which are based on $k$ and $k+l$ observations with an overlap of $k$ observations differ by at most $l / k)$. Similarly, it can be shown that for any $\varepsilon>0$

$$
\sup _{\lambda \geq \varepsilon} \sup _{u}\left|\hat{F}_{\left\lfloor n \theta_{0}\right\rfloor+1:\left\lfloor n \theta_{0}\right\rfloor+\left\lfloor n \lambda\left(1-\theta_{0}\right)\right\rfloor}(u)-\hat{F}_{\lfloor n \hat{\theta}\rfloor+1:\lfloor n \hat{\theta}\rfloor+\lfloor n \lambda(1-\hat{\theta})\rfloor}(u)\right|=O_{\mathbb{P}}\left(\left|\theta_{0}-\hat{\theta}\right|\right)=O_{\mathbb{P}}\left(n^{-1 / 2}\right),
$$

and the two displays above imply the weak convergence

$$
\begin{aligned}
\left(\left\{\lambda \sqrt{n}\left(\hat{F}_{1:\lfloor n \hat{\theta} \lambda\rfloor}(u)-F_{\mathbb{P}}(u)\right)\right\}_{\lambda \in[\varepsilon, 1], u \in \mathbb{R}^{d}},\left\{\lambda \sqrt{n}\left(\hat{F}_{\lfloor n \hat{\theta}\rfloor+1:\lfloor n \hat{\theta}\rfloor+\lfloor n \lambda(1-\hat{\theta})\rfloor}(u)-F_{\mathbb{Q}}(u)\right)\right\}_{\lambda \in[\varepsilon, 1], u \in \mathbb{R}^{d}}\right) \\
\rightsquigarrow\left(\left\{\mathbb{G}_{1}(\lambda, u)\right\}_{\lambda \in[\varepsilon, 1], u \in \mathbb{R}^{d}},\left\{\mathbb{G}_{2}(\lambda, u)\right\}_{\lambda \in[\varepsilon, 1], u \in \mathbb{R}^{d}}\right)
\end{aligned}
$$

in $\ell^{\infty}\left([\varepsilon, 1] \times \mathbb{R}^{d}\right)^{2}$. Assume that the mapping $\Phi$ is Hadamard differentiable at the points $F_{\mathbb{P}}, F_{\mathbb{Q}}$, tangentially to a vector space $V \subset \ell^{\infty}\left(\mathbb{R}^{d}\right)$, with linear derivatives $d \Phi_{F_{\mathbb{P}}}, d \Phi_{G_{\mathbb{P}}}$ and that $\mathbb{G}_{1}(\lambda, \cdot), \mathbb{G}_{2}(\lambda, \cdot) \in V$ a.s. Then the results in Section 4 of Volgushev and Shao (2014) combined with linearity of $d \Phi_{F_{\mathbb{P}}}, d \Phi_{F_{\mathbb{Q}}}$ and some calculations show that the weak convergence in (C.7) holds for the functional $\mu=\Phi(F)$ with $\hat{\mu}_{i: j}=\Phi\left(\hat{F}_{i: j}\right)$ provided that $\Lambda_{\nu} \subset[\varepsilon, 1]$. In particular we have have $\mathbb{H} \stackrel{\mathcal{D}}{=} \sigma^{2} \mathbb{B}$ for some $\sigma^{2}>0$. 